\title{
Comprehensive Taxonomies of Nature- and Bio-inspired Optimization: Inspiration versus Algorithmic Behavior, Critical Analysis and Recommendations
}

\author{
Daniel Molina*1, Javier Poyatos $^{1}$, Javier Del Ser ${ }^{2,3,4}$, Salvador García ${ }^{1}$, \\ Amir Hussain ${ }^{5}$, and Francisco Herrera ${ }^{1,6}$ \\ ${ }^{1}$ Department of Computer Science, DaSCI Andalusian Institute of Data Science and Computation \\ Intelligence, University of Granada, Spain, \{dmolina@ decsai.ugr.es, jpoyatosamador@ugr.es, \\ salvagl@decsai.ugr.es, herrera@ decsai.ugr.es\} \\ 2 TECNALIA, Spain, javier.delser@tecnalia.com \\ ${ }^{3}$ Dept. of Communications Engineering, University of the Basque Country (UPV/EHU), Spain \\ ${ }^{4}$ Basque Center for Applied Mathematics (BCAM), Spain \\ ${ }^{5}$ Edinburgh Napier University, United Kingdom, a.hussain@ napier.ac.uk \\ ${ }^{6}$ Faculty of Computing and Information Technology, King Abdulaziz University, Jeddah, Saudi Arabia
}

\begin{abstract}
In recent years, a great variety of nature- and bio-inspired algorithms has been reported in the literature. This algorithmic family simulates different biological processes observed in Nature in order to efficiently address complex optimization problems. In the last years the number of bio-inspired optimization approaches in literature has grown considerably, reaching unprecedented levels that dark the future prospects of this field of research. This paper addresses this problem by proposing two comprehensive, principle-based taxonomies that allow researchers to organize existing and future algorithmic developments into well-defined categories, considering two different criteria: the source of inspiration and the behavior of each algorithm. Using these taxonomies we review more than three hundred publications dealing with nature-inspired and bio-inspired algorithms, and proposals falling within each of these categories are examined, leading to a critical summary of design trends and similarities between them, and the identification of the most similar classical algorithm for each reviewed paper. From our analysis we conclude that a poor relationship is often found between the natural inspiration of an algorithm and its behavior. Furthermore, similarities in terms of behavior between different algorithms are greater than what is claimed in their public disclosure: specifically, we show that more than one-third of the reviewed bio-inspired solvers are versions of classical algorithms. Grounded on the conclusions of our critical analysis, we give several recommendations and points of improvement for better methodological practices in this active and growing research field.
\end{abstract}

Keywords - Nature-inspired algorithms, bio-inspired optimization, taxonomy, classification.

*Corresponding author 


\section{Introduction}

In many real-world optimization problems, no exact solver can be applied to solve them at an affordable computational cost or within a reasonable time, due to their complexity or the amount of data to use. In such cases the use of traditional techniques has been widely proven to be unsuccessful, thereby calling for the consideration of alternative optimization approaches.

In this context, complexity is not unusual in Nature: a plethora of complex systems, processes and behaviors have evinced a surprising performance to efficiently address intricate optimization tasks. The most clear example can be found in the different animal species, which have developed over generations very specialized capabilities by virtue of evolutionary mechanisms. Indeed, evolution has allowed animals to adapt to harsh environments, foraging, very difficult tasks of orientation, and to resiliently withstand radical climatic changes, among other threats. Animals, when organized in independent systems, groups or swarms or colonies (systems quite complex in their own) have managed to colonize the Earth completely, and eventually achieve a global equilibrium that has permitted them to endure for thousands of years.

This renowned success of biological organisms has inspired all kinds of solvers for optimization problems, which have been so far referred to as bio-inspired optimization algorithms. This family of optimization methods simulate biological processes such as natural evolution, where solutions are represented by individuals that reproduce and mutate to generate new, potentially improved candidate solutions for the problem at hand. Similarly, some other bio-inspired algorithms hinge on mimicking a collective behavior springing from the collaboration and interaction between simple agents, giving rise to the concept of Swarm Intelligence [1]. These are inspired by different biological animals: the movement of birds [2], bats [3], small insects such as fireflies [4], grasshoppers [5]; mechanisms to locate food exhibited by colony animals such as ants in Ant Colony Optimization (ACO, [6, 7]), or bees in Artificial Bee Colony algorithms (ABC, [8]); hunting mechanisms used by different animals, from small ones such as dragonflies [9], to wild wolves [10] or marine animals such as dolphins [9] or whales [11]; or other assorted biological phenomena such as the reproduction of coral reefs [12], the behavior of very small animals such as krill [13] or bacteria [14], to name a few. Inspiration in Nature could also stem from the observation and study of physical processes, without any biological motif. This is the case of rain [15], electromagnetism [16, 17], astronomy concepts like planets [18] or galaxies [19, 20], music [21], gases movement [22], or thermodynamics [23], among others.

More recently, algorithms inspired in human activities have also entered the scene, like sports [24, 25], decision making [26], or political systems $[27,28]$.

Disregarding their source of inspiration, there is clear evidence of the increasing popularity and notoriety gained by nature- and bio-inspired optimization algorithms in the last two decades. This momentum finds its reason in the capability of these algorithms to learn, adapt and provide good solutions to complex problems that otherwise would have remained unsolved. Many overviews have capitalized on this spectrum of algorithms applied to a wide range of problem casuistry, from combinatorial [29] to large-scale optimization [30], evolutionary data mining [1] and other alike. As a result, almost all 
business sectors have leveraged this success in recent times.

From a design perspective, nature- and bio-inspired optimization algorithms are usually conceived after observing a natural process or the behavioral patterns of biological organisms, which are then converted into a computational optimization algorithm. New discoveries in Nature and the undoubted increase of worldwide investigation efforts have ignited the interest of the research community in biological processes and their extrapolation to computational problems. As a result, many new bio-inspired meta-heuristics have appeared in the literature, unchaining an outbreak of proposals and applications every year. Nowadays, every natural process can be thought to be adaptable and emulated to produce a new meta-heuristic approach, yet with different capabilities of reaching global optimum solutions to optimization problems.

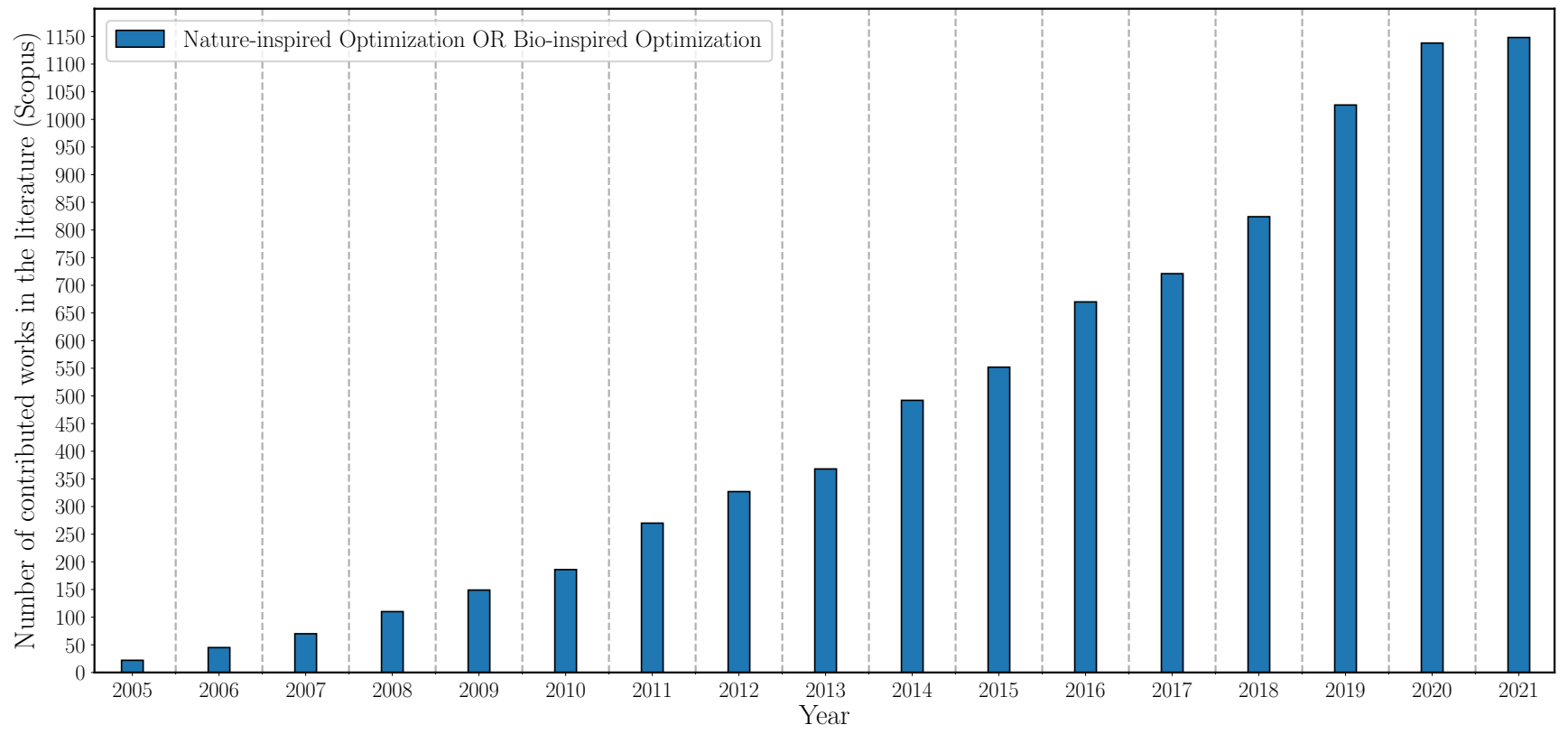

Figure 1: Number of papers with bio-inspired optimization and nature-inspired optimization in the title, abstract and/or keywords, over the period 2005- 2019 (Scopus database).

The above statement is quantitatively supported by Figure 1, which depicts the increasing number of papers/book chapters published in the last years with bio-inspired optimization and nature-inspired optimization in their title, abstract and/or keywords. We have considered both bio-inspired and nature-inspired optimization because sometimes both terms are confused and indistinctly used, although the nature-inspiration includes bio-inspired inspiration and complements it with other sources of inspirations (like physics-based optimization, chemistry-based optimization, ...). A major fraction of the publications comprising this plot proposed new bio-inspired algorithms at their time. From this rising number of nature and bio-inspired algorithms one can easily conclude that it would convenient to organize them into a taxonomy with well-defined criteria where to classify both the existing bio-inspired algorithms and new proposals to appear in the future. Unfortunately, the majority of such publications do not include any type of taxonomy, nor do they perform an exhaustive analysis of the similarity of 
their proposed algorithms with respect to other counterparts. Instead, they only focus on the nature or biological metaphor motivating the design of their meta-heuristic. This metaphor-driven research trend has been already denounced in several contributions [31, 32, 33], which have unleashed hot debates around specific meta-heuristic schemes that remain unresolved to date $[34,35]$. It is our firm belief that this controversy could be lessened by a comprehensive taxonomy of nature and bio-inspired optimization algorithms that settled the criteria to justify the novelty and true contributions of current and future advances in the field.

In this paper we have analyzed more than four hundred papers of different types of meta-heuristics and using that knowledge we present two different taxonomies for nature- and bio-inspired optimization algorithms:

- The first taxonomy classifies algorithms based on its natural or biological inspiration, so that given a specific algorithm, we can find its category quickly and without any ambiguity. The goal of this first taxonomy is to allow easily group the upsurge of solvers published in the literature.

- The second taxonomy classifies the reviewed algorithms based exclusively on their behavior, i.e., how they generate new candidate solutions for the function to be optimized. Our aim is to group together algorithms with a similar behavior, without considering its inspirational metaphor.

We believe that this dual criterion can be very useful for researchers. The first one helps classify the different proposals by its origin of inspiration, whereas the second one provides valuable information about their algorithmic similarities and differences. This double classification allows researchers to identify each new proposal in the adequate context. To the best of our knowledge, there has been no previous attempt as ambitious as the one presented in this overview to organize the existing literature on nature- and bio-inspired optimization.

Considering the classifications obtained in our wide study, we have critically examined the reviewed literature classification in the different taxonomies proposed in this work. The goal is to analyze if there is a relationship between the algorithms classified in a same category in one category and the classification on the other taxonomy. Next, similarities detected among algorithms will allow us to discover the most influential meta-heuristics, whose behavior has inspired many other nature- and bio-inspired proposals.

Finally, we do a critical analysis and provide several recommendations towards improving research practices in this field. The growing number of nature-inspired proposals could be seen as a symptom of the active status of this field; however, its sharp evolution suggests that research efforts should be also invested towards new behavioral differences and verifiable performance evidences in practical problems.

The rest of this paper is organized as follows. In Section 2, we examine previous surveys, taxonomies and reviews of nature- and bio-inspired algorithms reported so far in the literature. Section 3 delves the taxonomy based on the inspiration of 
the algorithms. In Section 4, we present and populate the taxonomy based on the behavior of the algorithm. In Section 5, we analyze similarities and differences found between both taxonomies, ultimately identifying the most influential algorithms in our reviewed papers. In Section 6, some conclusions and suggestions for improvement are given, remarking that the behavior of algorithms is more relevant than their natural inspiration. We thereby encourage researchers to be more focused on applying these algorithms to more problems, and to participate in competitions to assess their good performance. Finally, in Section 7 , we summarize our main conclusions.

\section{Related Literature Studies}

The diversity of bio-inspired algorithms and their flexibility to tackle optimization problems for many research fields have inspired several surveys and overviews to date. Most of them have focused on different types of problems [36, 37], including continuous [38], combinatorial [29], or multi-objective optimization problems [39]. For specific real-world problems, the prolific literature about nature- and bio-inspired algorithms has sparked specific state-of-the-art studies revolving on their application to different fields, such as Telecommunications [40], Robotics [41], Data Mining [42], Structural Engineering [39] or Transportation [43]. Even specific real-world problems have been dedicated exclusive overviews due to the vast research reported around the topic, like power systems [44], the design of computer networks [45], automatic clustering [46], face recognition [47], molecular docking [48], or intrusion detection [49], to mention a few.

Seen from the algorithmic perspective, many particular bio-inspired solvers have been modified along the years to yield different versions analyzed in surveys devoted to that type of algorithms, from classical approaches such as PSO [50] and DE [51, 52, 53] to more modern ones, e.g., ABC [54, 55], Bacterial Foraging Optimization Algorithm (BFOA, [56]) or the Bat Algorithm [57]. From a more general albeit still algorithmic point of view, [31] explains how the metaphor and the biological idea is used to create a bio-inspired meta-heuristic optimization algorithm. In this study the reader is also provided with some examples and characteristics of this design process. Books like [58] or [59] show many nature-inspired algorithms. However, they are more focused on describing the different algorithms available in the literature than on classifying and analyzing them in depth.

Several comparison studies among bio-inspired algorithms with very different behaviors can be found in the current literature, which mostly aim at deciding which approach to use for solving a problem. In [60], the popular PSO and DE versions are compared. This research line is followed by [61], which compared the performance of different bio-inspired algorithms, again with prescribing which one to use as its primary goal. More recently, [62] examined the features of several recent bio-inspired algorithms, suggesting, on a concluding note, to which type of problem each of the examined algorithms should be applied. More specific is the work in [63], which compares several different algorithms considering 
its parallel implementation on GPU devices. More recently, the focus has shifted towards comparing groups of algorithms instead of making a comparison between individual solvers: this is the case of [64], which compares Swarm Intelligence and Evolutionary Computation methods in order to assess their properties and behavior (e.g., their convergence speed). Once again, the main purpose of this recent literature strand is to compare bio-inspired algorithms, not to classify them nor to find similarities and design patterns among them. In [65], foraging algorithms (such as the aforementioned BFOA) are compared against other evolutionary algorithms. In that paper, algorithms are classified in two large groups: algorithms with and without cooperation. In [66, 67], PSO was proven to outperform other bio-inspired approaches (namely, DE, GA and ABC) when used for efficiently training and configuring Echo State Networks.

It has not been until relatively recent times when the community has embraced the need for arranging the myriad of existing bio-inspired algorithms and classifying them under principled, coherent criteria. In 2013, [68] presented a classification of meta-heuristic algorithms as per their biological inspiration that discerned categories with similar approaches in this regard: Swarm Intelligence, Physics and Chemistry Based, Bio-inspired algorithms (not SI-based), and an Other algorithms category. However, the classification given in this paper is not actually hierarchical, so it can not be regarded as a true taxonomy. Another classification was proposed in [69, 70], composed by Evolution Based Methods, Physics Based Methods, Swarm Based Methods, and Human-Based Methods. With respect to the preceding classification, a new Human-Based category is proposed, which collectively refers to algorithms inspired in the human behavior. The classification criteria underneath these categories is used to build up a catalog of more than 50 algorithmic proposals, obtaining similar groups in size. In this case, there is no miscellaneous category as in the previous classification. Similarly to [68], categories are disjoint groups without subcategories.

Recently, [71] offers a review of meta-heuristics from the 1970s until 2015, i.e, from the development of neural networks to novel algorithms like Cuckoo Search. Specifically a broad view of new proposals is given, but without proposing any category. The most recent survey to date is that in [72], in which nature-inspired algorithms are classified not in terms of their source of inspiration, but rather by their behavior. Consequently, algorithms are classified as per three different principles. The first one is learning behavior, namely, how solutions are learned from others preceding them. The learning behavior can be individual, local (i.e., only inside a neighborhood), global (between all individuals), and none (no learning). The second principle is interaction-collective behavior, denoting whether individuals cooperate or compete between them. The third principle is referred to as diversification-population control, by which algorithms are classified based on whether the population has a converging tendency, a diffuse tendency, or no specific tendency. Then, 29 bio-inspired algorithms are classified by each of these principles, and approaches grouped by each principle are experimentally compared.

The prior related work reviewed above indicates that the community widely acknowledges (with more emphasis in recent times) the need for properly organizing the plethora of bio- and nature-inspired algorithms in a coherent taxonomy. However, 
the majority of them are only focused on the natural inspiration of the algorithms for creating the taxonomy, not giving any attention to their behavior. Only [72] considers this aspect, but does not propose a real taxonomy, but rather different independent design principles. On the contrary, as will be next described, our proposed taxonomies consider 1) the source of inspiration; and 2) the procedure by which new solutions are produced over the search process of every algorithm (behavior). Furthermore, we note that efforts invested in this regard to date are not up to the level of ambition and thoroughness pursued in our study. In addition, no study published so far has been specifically devoted to unveiling structural similarities among classical and modern meta-heuristics. There lies the novelty and core contribution of our study, along with the aforementioned novel behavior-based taxonomy.

\section{Taxonomy by Source of Inspiration}

In this section, we propose a novel taxonomy based on the inspirational source in which nature- and bio-inspired algorithms are claimed to find their design rationale in the literature. This allows classifying the great amount and variety of contributions reported in related fora.

The proposed taxonomy presented in what follows was developed reviewing more than 400 papers over different years, starting from the most classical proposals in the late 80's (such as Simulated Annealing [23] or PSO [2]) to more novel techniques appearing in the literature until 2021 [73]. Thus, to our knowledge, this exercise can be considered the most exhaustive review in the area to date.

Taking in account all the reviewed papers, we group the proposals therein in a hierarchy of categories. In the hierarchy, not all proposals of a category must fit in one of its subcategories. In our classification, categories laying at the same level are disjoint sets, which involves that each proposed algorithm can be only a member of one of these categories. To this end, for each algorithm we select the category considered to be most suitable considering the nuances of the algorithm that allow us to differentiate it from its remaining counterparts.

Methodologically, a classification of all nature- and bio-inspired algorithms that can be found in the literature can become complicated, considering the different sources of inspiration as biological, physical, human-being, ... In some papers, authors suggest a possible categorization of more well-established groups, but not in all of them. Also, its classification could not be the more appropriate and become eventually obsolete, since the number of proposals - and thereby, the diversity of sources of inspiration motivating them - increases over time. Algorithms within each proposed category were selected by their relative importance in the field, considering the number of citations, the number of algorithmic variants that were inspired by that algorithm, and other similar factors.

When establishing a hierarchical classification, it is important to achieve a good trade-off between information and 
simplicity by the following criteria:

- When to establish a new division of a category into subcategories: a coarse split criterion for the taxonomy can imply categories of little utility for the subsequent analysis, since in that case, the same category would group very different algorithms. On the other hand, a fine-grained taxonomy can produce very complex hierarchies and, therefore, with little usefulness. For keeping the taxonomy simple yet informative for our analytical purposes, we decided that a category should have at least four algorithms in order to be kept in the taxonomy. Thus, a category is only decomposed in subcategories if each of them has coherence and a minimum representativeness (as per the number of algorithms it contains).

- Which number of subcategories into which to divide a category: the criterion followed in this regard must produce meaningful subcategories. In order to ensure a reduced number of subcategories, we consider that not all algorithms inside one category must be a member of one of its subcategories. In that way, we avoid introducing mess categories that lack cohesion.

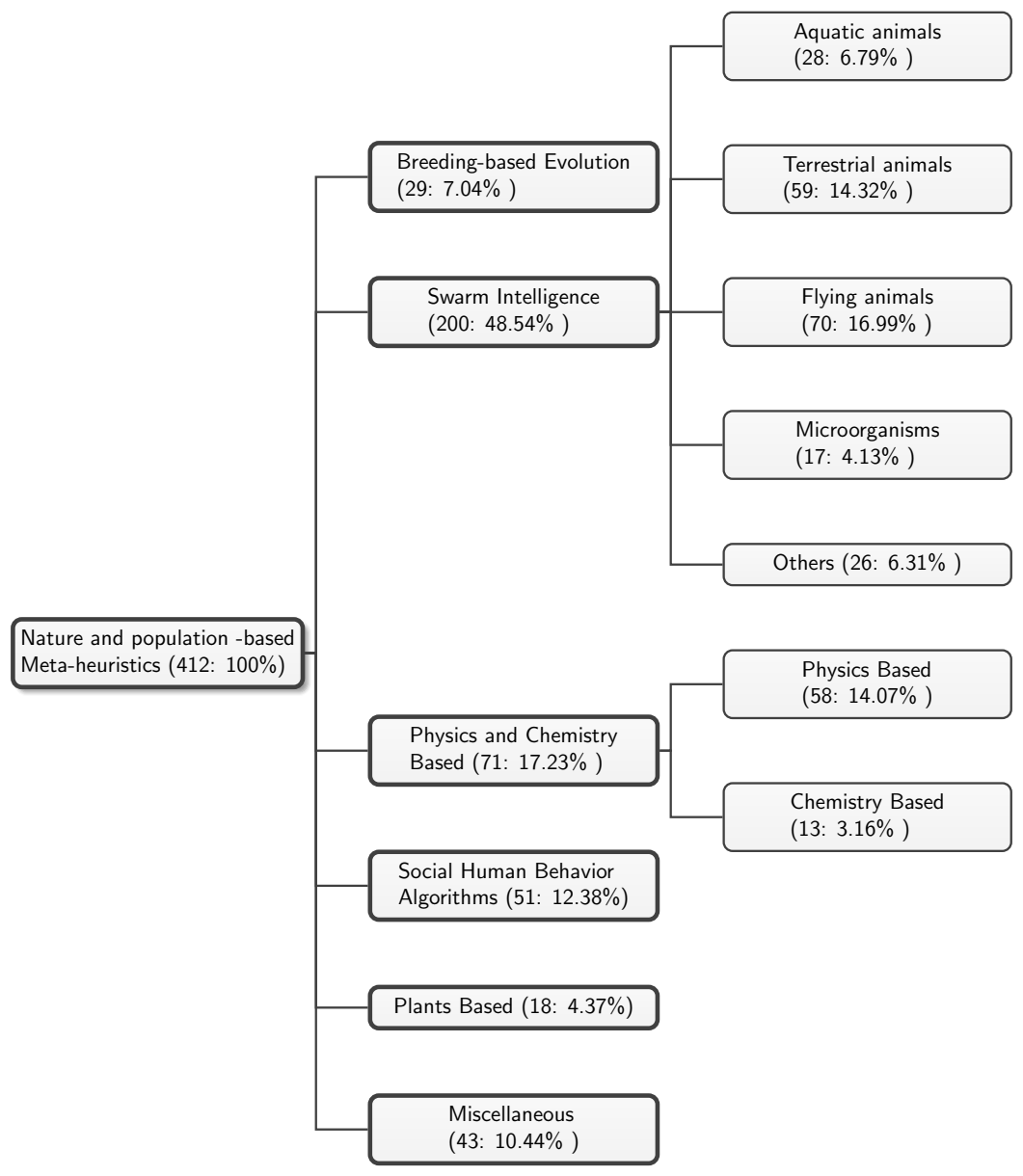

Figure 2: Classification of the reviewed papers using the inspiration source based taxonomy. 


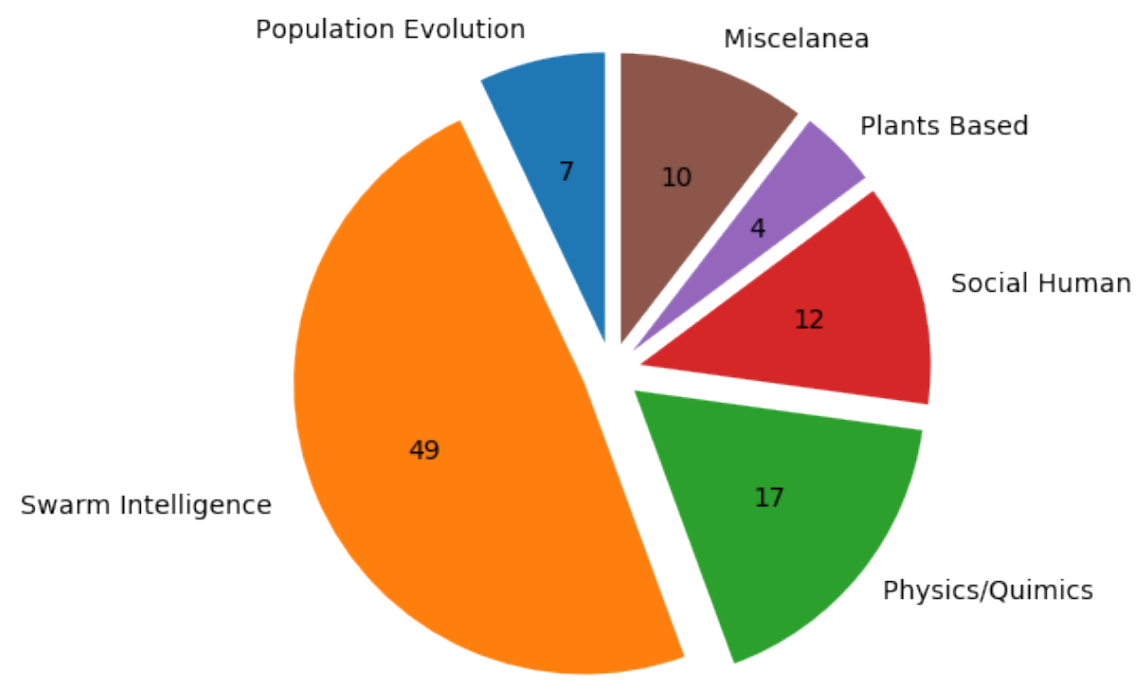

Figure 3: Ratio of reviewed algorithms by its category (first taxonomy).

Figure 2 depicts the classification we have reached, indicating, for the more than 400 reviewed algorithms, the number and ratio of proposals classified in each category and subcategory. It can be observed that the largest group of all is Swarm Intelligence category (near the half of the proposed, 48\%), inspired in the Swarm Intelligence concept [58], followed by the Physics and Chemistry category, inspired by different physical behaviors or chemical reactions (17\% of proposals). Other relevant categories are Social Human Behavior Algorithms (12\%), inspired by human aspects, and Breeding-based Evolution (7\%), inspired by the Theory of Evolution over a population of individuals, that includes very renowned algorithms such as Genetic Algorithms. A new category emerges from our study - Plants Based - which has not been included in other taxonomies. Nearly $4 \%$ of proposals are so different between them that they cannot be grouped in new categories. The percentage of classification of each category is visually displayed in Figure 3.

For the sake of clarity regarding the classification criteria, in the next subsections we provide a brief description of the different categories in this first taxonomy, including their main characteristics, an example, and a table listing the algorithms belonging to each category.

\subsection{Breeding-based Evolutionary Algorithms}

This category comprises population-based algorithms inspired in the principles of Natural Evolution. Each individual in the population represents a solution of the problem, and has an associated fitness value (namely, the value of the problem objective 
function for that solution). In these algorithms, a process of reproduction (also referred to breeding or crossover) and survival iterated over successive generations makes the population of solutions potentially evolve towards regions of higher optimality (as told by the best solution in the population). Thus, this category is characterized by the fact of being inspired by the concept of breeding-based evolution, without considering other operators performed on individuals than reproduction (e.g., mutation).

More in detail, in these algorithms we have a population with individuals that have the ability to breed and produce new offspring. Therefore, from the parents we get children, which introduce some variety with respect to their parents. These characteristics allow them to adapt to the environment which, translated to the optimization realm, permits to search more efficiently over the solution space of the problem at hand. By virtue of this mechanism we have a population that evolves through generations and, when combined with a selection (survival) and - possibly - other operators, results are improved. Nevertheless, the breeding characteristic is what makes algorithms within this category unique with respect to those in other categories.

Table 1 compiles all reviewed algorithms that fall within this category. As could have been a priori expected, well-known classical Evolutionary Computation algorithms can be observed in this list, such as Genetic Algorithm (GA), Evolution Strategies (ES) and Differential Evolution (DE). However, other algorithms based in the reproduction of different biological organisms are also notable, such as queen bees and weeds.

\subsection{Swarm Intelligence based Algorithms}

Swarm Intelligence (SI) is already a consolidated term in the community, which was first introduced by Gerardo Beni and Jing Wang in 1989 [41]. It can be defined as the collective behavior of decentralized, self-organized systems, in either natural or artificial environments. The expression was proposed in the context of robotic systems, but has generalized over the years to denote the emergence of collective intelligence from a group of simple agents, governed by simple behavioral rules. Thus, bio-inspired meta-heuristics based on Swarm Intelligence are those inspired on the collective behavior of animal societies, such as insect colonies or bird flocks, wherein the collective intelligence emerging from the swarm permits to efficiently undertake optimization problems. The seminal bio-inspired algorithm relying on SI concepts was PSO [2], followed shortly thereafter by ACO [6]. These early findings around SI concepts applied to optimization spurred many SI-based algorithms in

subsequent years, such as ABC [8] and more recently, Firefly Algorithm (FA, [4]) and Grasshopper Optimization Algorithm (GOA, [5]).

Reviewed algorithms that fall under the Swarm Intelligence umbrella are shown in Tables 2, 3, 4, 5 and 6. This is the most populated category of all our study, characterized by a first category that relates to the type of animal that have inspired each algorithm: as such, we find i) flying animals, namely, algorithms inspired in the flying movement of birds and flying animals like insects; ii) terrestrial animals, inspired by the foraging and hunter mechanisms of land animals; iii) aquatic animals, 
Table 1: Nature- and bio-inspired meta-heuristics within the Breeding-based Evolution category.

\section{Breeding-based Evolution}

\begin{tabular}{lllr}
\hline Algorithm Name & Acronym & Year & Reference \\
\hline Artificial Ecosystem Algorithm & AEA & 2014 & {$[74]$} \\
Artificial Infections Disease Optimization & AIDO & 2016 & {$[75]$} \\
Asexual Reproduction Optimization & ARO & 2010 & {$[76]$} \\
Biogeography Based Optimization & BBO & 2008 & {$[77]$} \\
Bird Mating Optimization & BMO & 2014 & {$[78]$} \\
Bean Optimization Algorithm & BOA & 2011 & {$[79]$} \\
Coral Reefs Optimization & CRO & 2014 & {$[12]$} \\
Dendritic Cells Algorithm & DCA & 2005 & {$[80]$} \\
Differential Evolution & DE & 1997 & {$[81]$} \\
Ecogeography-Based Optimization & EBO & 2014 & {$[82]$} \\
Eco-Inspired Evolutionary Algorithm & EEA & 2011 & {$[83]$} \\
Earthworm Optimization Algorithm & EOA & 2018 & {$[84]$} \\
Evolution Strategies & ES & 2002 & {$[85]$} \\
Genetic Algorithms & GA & 1989 & {$[86]$} \\
Gene Expression & GE & 2001 & {$[87]$} \\
Hybrid Rice Optimization & HRO & 2016 & {$[88]$} \\
Immune-Inspired Computational Intelligence & ICI & 2008 & {$[89]$} \\
Improved Genetic Immune Algorithm & IGIA & 2017 & {$[90]$} \\
Weed Colonization Optimization & IWO & 2006 & {$[91]$} \\
Marriage In Honey Bees Optimization & MHBO & 2001 & {$[92]$} \\
Mushroom Reproduction Optimization & MRO & 2018 & {$[93]$} \\
Queen-Bee Evolution & QBE & 2003 & {$[94]$} \\
SuperBug Algorithm & SuA & 2012 & {$[95]$} \\
Stem Cells Algorithm & SCA & 2011 & {$[96]$} \\
Sheep Flock Heredity Model & SFHM & 2001 & {$[97]$} \\
Swine Influenza Models Based Optimization & SIMBO & 2013 & {$[98]$} \\
Self-Organizing Migrating Algorithm & SOMA & 2004 & {$[99]$} \\
Variable Mesh Optimization & VMO & 2012 & {$[100]$} \\
Virulence Optimization Algorithm & VOA & 2016 & {$[101]$} \\
\hline
\end{tabular}


whose inspiration emerges from the movement of fish schools or other aquatic animals like dolphins; and iv) microorganisms, inspired by virus, bacteria, algae and others alike.

Inside each subcategory, we have also distinguished whether they are inspired by the foraging action of the inspired living creature - Foraging-inspired is in fact another popular term related to this type of inspiration [102] - or, instead, by its movement patterns in general. When the behavior of the algorithm is inspired in both the movement and the foraging behavior of the animal, it is cataloged as foraging inside our first taxonomy. We will next examine in depth each of these subcategories.

\subsubsection{Subcategories of SI based algorithms by the environment}

As mentioned previously, the global set of Swarm Intelligence algorithms can be divided as a function of the type of animals. Between the possible categories stemming from this criteria, we have grouped them according to the environmental medium inhibited by the inspiring animal (aquatic, terrestrial or aerial). This criterion is not only very intuitive, since it inherits a criterion already applied in animal taxonomies, but it also relies on the fact that these environments condition the movement and hunting mode of the different species. As such, the following subcategories have been established:

- Flying animals: This category comprises meta-heuristics based on the concept of Swarm Intelligence in which the trajectory of agents is inspired by flying movements, as those observed in birds, bats, or other flying insects. The most well-known algorithm in this subcategory are PSO [2] and ABC [8].

- Terrestrial animals: Meta-heuristics in this category are inspired by foraging or movements in terrestrial animals. The most renowned approach within this category is the classical ACO meta-heuristic [6], which replicates the stigmergic mechanism used by ants to locate food sources and inform of their existence to their counterparts in the colony. This category also includes other popular algorithms like Grey Wolf Optimization (GWO, [185]), inspired in the wild wolf hunting strategy, Lion Optimization Algorithm (LOA, [205]), which imitates hunting methods used by these animals, or the more recent Grasshopper Optimization Algorithm (GOA, [5]), which finds its motivation in the jumping motion of grasshoppers.

- Aquatic animals: This type of meta-heuristic algorithms focuses on aquatic animals. The aquatic ecosystem in which they live have inspired different exploration mechanisms. It contains popular algorithms as Krill Herd (KH, [13]), Whale Optimization Algorithm (WOA, [11]), and algorithms based on the echolocation used by dolphins to detect fish like Dolphin Partner Optimization (DPO, [163]) and Dolphin Echolocation [161].

- Microorganisms: Meta-heuristics based on microorganisms are related with the food search performed by bacteria. A bacteria colony performs a movement to search for food. Once they have found and taken it, they split to search again in the environment. Other types of meta-heuristics that can be part of this category are the ones related with virus, which 
Table 2: Nature- and bio-inspired meta-heuristics within the Swarm Intelligence category (I).

\begin{tabular}{|c|c|c|c|c|c|}
\hline \multicolumn{6}{|c|}{ Swarm Intelligence (I) } \\
\hline Algorithm Name & Acronym & Subcategory & Type & Year & Reference \\
\hline Artificial Algae Algorithm & AAA & Micro & Movement & 2015 & [103] \\
\hline Artificial Beehive Algorithm & $\mathrm{ABA}$ & Flying & Foraging & 2009 & [104] \\
\hline Artificial Bee Colony & $\mathrm{ABC}$ & Flying & Foraging & 2007 & [8] \\
\hline Animal Behavior Hunting & $\mathrm{ABH}$ & Other & Foraging & 2014 & [105] \\
\hline African Buffalo Optimization & $\mathrm{ABO}$ & Terrestrial & Foraging & 2016 & [106] \\
\hline Andean Condor Algorithm & $\mathrm{ACA}$ & Flying & Foraging & 2019 & [107] \\
\hline Ant Colony Optimization & $\mathrm{ACO}$ & Terrestrial & Foraging & 1996 & [6] \\
\hline Artificial Feeding Birds & AFB & Flying & Movement & 2018 & [108] \\
\hline Animal Migration Optimization & AMO & Other & Movement & 2014 & [109] \\
\hline Ant Lion Optimizer & ALO & Terrestrial & Foraging & 2015 & [110] \\
\hline Anglerfish Algorithm & $\mathrm{AOA}$ & Aquatic & Movement & 2019 & [111] \\
\hline Artificial Searching Swarm Algorithm & ASSA & Other & Movement & 2009 & [112] \\
\hline Artificial Tribe Algorithm & ATA & Other & Movement & 2009 & [113] \\
\hline African Wild Dog Algorithm & AWDA & Terrestrial & Foraging & 2013 & {$[114]$} \\
\hline Bald Eagle Search & BES & Flying & Foraging & 2019 & [115] \\
\hline Bees Algorithm & BA & Flying & Foraging & 2006 & [116] \\
\hline Bumblebees & BB & Flying & Foraging & 2009 & [117] \\
\hline Bison Behavior Algorithm & BBA & Terrestrial & Movement & 2019 & [118] \\
\hline Bee Colony-Inspired Algorithm & BCIA & Flying & Foraging & 2009 & [119] \\
\hline Bee Colony Optimization & $\mathrm{BCO}$ & Flying & Foraging & 2005 & [120] \\
\hline Bacterial Colony Optimization & BCO.1 & Micro & Foraging & 2012 & [121] \\
\hline Bacterial Chemotaxis Optimization & BCO.2 & Micro & Foraging & 2002 & [122] \\
\hline Border Collie Optimization & BCO.3 & Terrestrial & Movement & 2020 & [123] \\
\hline $\begin{array}{l}\text { Biomimicry Of Social Foraging Bacteria for } \\
\text { Distributed Optimization }\end{array}$ & BFOA & Micro & Foraging & 2002 & [14] \\
\hline Bacterial Foraging Optimization & BFOA.1 & Micro & Foraging & 2009 & [56] \\
\hline Bacterial-GA Foraging & BGAF & Micro & Foraging & 2007 & [124] \\
\hline BeeHive Algorithm & BHA & Flying & Foraging & 2004 & [125] \\
\hline Bees Life Algorithm & BLA & Flying & Foraging & 2018 & [126] \\
\hline Bat Intelligence & BI & Flying & Foraging & 2012 & [127] \\
\hline Bat Inspired Algorithm & BIA & Flying & Foraging & 2010 & [3] \\
\hline Biology Migration Algorithm & BMA & Other & Movement & 2019 & [128] \\
\hline Barnacles Mating Optimizer & BMO.1 & Micro & Movement & 2019 & [129] \\
\hline Blind, Naked Mole-Rats Algorithm & BNMR & Terrestrial & Foraging & 2013 & [130] \\
\hline Butterfly Optimizer & $\mathrm{BO}$ & Flying & Movement & 2015 & [131] \\
\hline Bonobo Optimizer & BO.1 & Terrestrial & Movement & 2019 & [132] \\
\hline Bull Optimization Algorithm & BOA.1 & Terrestrial & Movement & 2015 & [133] \\
\hline Bee System & BS & Flying & Foraging & 1997 & [134] \\
\hline Bee System & BS.1 & Flying & Foraging & 2002 & [135] \\
\hline Bird Swarm Algorithm & BSA & Flying & Movement & 2016 & [136] \\
\hline Bee Swarm Optimization & BSO & Flying & Foraging & 2010 & [137] \\
\hline Bioluminiscent Swarm Optimization Algorithm & BSO.1 & Flying & Foraging & 2011 & [138] \\
\hline Bees Swarm Optimization Algorithm & BSOA & Flying & Foraging & 2005 & [139] \\
\hline Buzzard Optimization Algorithm & BUZOA & Flying & Foraging & 2019 & [140] \\
\hline Black Widow Optimization Algorithm & BWO & Terrestrial & Movement & 2020 & [141] \\
\hline Binary Whale Optimization Algorithm & BWOA & Aquatic & Foraging & 2019 & [142] \\
\hline
\end{tabular}


Table 3: Nature- and bio-inspired meta-heuristics within the Swarm Intelligence category (II).

\begin{tabular}{|c|c|c|c|c|c|}
\hline \multicolumn{6}{|c|}{ Swarm Intelligence (II) } \\
\hline Algorithm Name & Acronym & Subcategory & Type & Year & Reference \\
\hline Collective Animal Behavior & $\mathrm{CAB}$ & Other & Foraging & 2012 & [143] \\
\hline Cheetah Based Algorithm & CBA & Terrestrial & Movement & 2018 & [144] \\
\hline Catfish Optimization Algorithm & $\mathrm{CAO}$ & Aquatic & Movement & 2011 & [145] \\
\hline Cricket Behavior-Based Algorithm & CBBE & Terrestrial & Movement & 2016 & [146] \\
\hline Cultural Coyote Optimization Algorithm & CCOA & Terrestrial & Movement & 2019 & [147] \\
\hline Chaotic Crow Search Algorithm & CCSA & Flying & Foraging & 2018 & [148] \\
\hline Chaotic Dragonfly Algorithm & CDA & Flying & Foraging & 2018 & [149] \\
\hline Cuttlefish Algorithm & CFA & Aquatic & Movement & 2013 & [150] \\
\hline Consultant Guide Search & CGS & Other & Movement & 2010 & [151] \\
\hline Camel Herd Algorithm & CHA & Terrestrial & Foraging & 2017 & [152] \\
\hline Cuckoo Optimization Algorithm & $\mathrm{COA}$ & Flying & Foraging & 2011 & [153] \\
\hline Camel Travelling Behavior & COA.1 & Terrestrial & Movement & 2016 & [154] \\
\hline Coyote Optimization Algorithm & COA.2 & Terrestrial & Movement & 2018 & [155] \\
\hline Cuckoo Search & $\mathrm{CS}$ & Flying & Foraging & 2009 & [156] \\
\hline Crow Search Algorithm & CSA & Flying & Movement & 2016 & [157] \\
\hline Chameleon Swarm Algorithm & CSA. 2 & Terrestrial & Foraging & 2021 & [73] \\
\hline Cat Swarm Optimization & $\mathrm{CSO}$ & Terrestrial & Movement & 2006 & [158] \\
\hline Chicken Swarm Optimization & CSO.1 & Terrestrial & Movement & 2014 & [159] \\
\hline Dragonfly Algorithm & DA & Flying & Foraging & 2016 & [9] \\
\hline Dragonfly Swarm Algorithm & DA.1 & Flying & Foraging & 2020 & [160] \\
\hline Dolphin Echolocation & DE.1 & Aquatic & Foraging & 2013 & [161] \\
\hline Deer Hunting Optimization Algorithm & DHOA & Terrestrial & Foraging & 2019 & [162] \\
\hline Dolphin Partner Optimization & DPO & Aquatic & Movement & 2009 & [163] \\
\hline Donkey Theorem Optimization & DTO & Terrestrial & Foraging & 2019 & [164] \\
\hline Elephant Herding Optimization & $\mathrm{EHO}$ & Terrestrial & Movement & 2016 & [165] \\
\hline Emperor Penguins Colony & EPC & Terrestrial & Movement & 2019 & [166] \\
\hline Emperor Penguin Optimizer & EPO & Terrestrial & Movement & 2018 & [167] \\
\hline Eagle Strategy & ES.1 & Flying & Foraging & 2010 & [168] \\
\hline Elephant Search Algorithm & ESA & Terrestrial & Foraging & 2015 & [169] \\
\hline Elephant Swarm Water Search Algorithm & ESWSA & Terrestrial & Movement & 2018 & [170] \\
\hline Egyptian Vulture Optimization Algorithm & $\mathrm{EV}$ & Flying & Foraging & 2013 & [171] \\
\hline Firefly Algorithm & FA & Flying & Foraging & 2009 & [4] \\
\hline Flocking Base Algorithms & FBA & Flying & Movement & 2006 & [172] \\
\hline Fast Bacterial Swarming Algorithm & FBSA & Micro & Foraging & 2008 & [173] \\
\hline Frog Call Inspired Algorithm & FCA & Terrestrial & Movement & 2009 & [174] \\
\hline Flock by Leader & FL & Flying & Movement & 2012 & [175] \\
\hline Fruit Fly Optimization Algorithm & FOA & Flying & Foraging & 2012 & [176] \\
\hline Falcon Optimization Algorithm & FOA.2 & Flying & Foraging & 2019 & [177] \\
\hline Fish-Swarm Algorithm & FSA & Aquatic & Foraging & 2002 & [178] \\
\hline Fish Swarm Algorithm & FSA.1 & Aquatic & Foraging & 2011 & [179] \\
\hline Fish School Search & FSS & Aquatic & Foraging & 2008 & [180] \\
\hline Group Escape Behavior & GEB & Aquatic & Movement & 2011 & [181] \\
\hline Good Lattice Swarm Optimization & GLSO & Other & Movement & 2007 & [182] \\
\hline Grasshopper Optimisation Algorithm & GOA & Terrestrial & Foraging & 2017 & [5] \\
\hline Glowworm Swarm Optimization & GSO & Micro & Movement & 2013 & [20] \\
\hline Group Search Optimizer & GSO.1 & Other & Movement & 2009 & [183] \\
\hline Goose Team Optimization & GTO & Flying & Movement & 2008 & [184] \\
\hline Grey Wolf Optimizer & GWO & Terrestrial & Foraging & 2014 & [185] \\
\hline
\end{tabular}


Table 4: Nature- and bio-inspired meta-heuristics within the Swarm Intelligence category (III).

\begin{tabular}{|c|c|c|c|c|c|}
\hline \multicolumn{6}{|c|}{ Swarm Intelligence (III) } \\
\hline Algorithm Name & Acronym & Subcategory & Type & Year & Reference \\
\hline Hitchcock Birds-Inspired Algorithm & HBIA & Flying & Movement & 2020 & [186] \\
\hline Honey-Bees Mating Optimization Algorithm & HBMO & Flying & Movement & 2006 & [187] \\
\hline Harry’s Hawk Optimization Algorithm & HHO & Flying & Foraging & 2019 & [188] \\
\hline Hoopoe Heuristic Optimization & HHO.1 & Flying & Foraging & 2012 & [189] \\
\hline Horse Optimization Algorithm & HOA & Terrestrial & Movement & 2020 & [190] \\
\hline Hunting Search & $\mathrm{HuS}$ & Other & Foraging & 2010 & [191] \\
\hline Honeybee Social Foraging & HSF & Flying & Foraging & 2007 & [192] \\
\hline Hierarchical Swarm Model & HSM & Other & Movement & 2010 & [193] \\
\hline Hammerhead Shark Optimization Algorithm & HSOA & Aquatic & Foraging & 2019 & [194] \\
\hline Hypercube Natural Aggregation Algorithm & HYNAA & Other & Movement & 2019 & [195] \\
\hline Improved Raven Roosting Algorithm & IRRO & Flying & Movement & 2018 & [196] \\
\hline Invasive Tumor Optimization Algorithm & ITGO & Micro & Movement & 2015 & [197] \\
\hline Jaguar Algorithm & $\mathrm{JA}$ & Terrestrial & Foraging & 2015 & [198] \\
\hline Japanese Tree Frogs Calling Algorithm & JTFCA & Terrestrial & Movement & 2012 & [199] \\
\hline Krill Herd & $\mathrm{KH}$ & Aquatic & Foraging & 2012 & [13] \\
\hline Krestrel Search Algorithm & KSA & Flying & Foraging & 2016 & [200] \\
\hline Killer Whale Algorithm & KWA & Aquatic & Foraging & 2017 & [201] \\
\hline Lion Algorithm & LA & Terrestrial & Foraging & 2012 & [202] \\
\hline Seven-Spot Labybird Optimization & LBO & Flying & Foraging & 2013 & [203] \\
\hline Laying Chicken Algorithm & LCA & Terrestrial & Movement & 2017 & [204] \\
\hline Lion Optimization Algorithm & LOA & Terrestrial & Foraging & 2016 & [205] \\
\hline Lion Pride Optimizer & LPO & Terrestrial & Movement & 2012 & [206] \\
\hline Locust Swarms Optimization & LSO & Aquatic & Foraging & 2009 & [207] \\
\hline Locust Swarms Search & LSS & Aquatic & Movement & 2015 & [208] \\
\hline Mayfly Optimization Algorithm & MA.1 & Flying & Movement & 2020 & [209] \\
\hline Magnetotactic Bacteria Optimization Algorithm & $\mathrm{MBO}$ & Micro & Movement & 2013 & [210] \\
\hline Monarch Butterfly Optimization & MBO.1 & Flying & Movement & 2017 & [211] \\
\hline Migrating Birds Optimization & MBO.2 & Flying & Movement & 2012 & [212] \\
\hline Mouth Breeding Fish Algorithm & MBF & Aquatic & Foraging & 2018 & [213] \\
\hline Modified Cuckoo Search & MCS & Flying & Foraging & 2009 & [214] \\
\hline Modified Cockroach Swarm Optimization & MCSO & Terrestrial & Foraging & 2011 & [215] \\
\hline Moth Flame Optimization Algorithm & MFO & Flying & Movement & 2015 & [216] \\
\hline Mosquito Flying Optimization & MFO.1 & Flying & Foraging & 2016 & [217] \\
\hline Meerkats Inspired Algorithm & MIA & Terrestrial & Movement & 2018 & [218] \\
\hline Mox Optimization Algorithm & MOX & Flying & Movement & 2011 & [219] \\
\hline Marine Predators Algorithm & MPA & Aquatic & Foraging & 2020 & [220] \\
\hline Monkey Search & MS & Terrestrial & Foraging & 2007 & [221] \\
\hline Natural Aggregation Algorithm & NAA & Other & Movement & 2016 & [222] \\
\hline Naked Moled Rat & NMR & Terrestrial & Movement & 2019 & [223] \\
\hline Nomadic People Optimizer & NPO & Other & Movement & 2019 & [224] \\
\hline Orcas Intelligence Algorithm & OA & Aquatic & Foraging & 2020 & [225] \\
\hline OptBees & OB & Flying & Foraging & 2012 & [226] \\
\hline Optimal Foraging Algorithm & OFA & Other & Foraging & 2017 & [227] \\
\hline Owls Optimization Algorithm & OOA & Flying & Movement & 2019 & [228] \\
\hline Pity Beetle Algorithm & PBA & Terrestrial & Foraging & 2018 & [229] \\
\hline Polar Bear Optimization Algorithm & PBOA & Terrestrial & Foraging & 2017 & [230] \\
\hline Pigeon Inspired Optimization & PIO & Flying & Movement & 2014 & [231] \\
\hline Population Migration Algorithm & PMA & Other & Movement & 2009 & [232] \\
\hline
\end{tabular}


Table 5: Nature- and bio-inspired meta-heuristics within the Swarm Intelligence category (IV).

\begin{tabular}{|c|c|c|c|c|c|}
\hline \multicolumn{6}{|c|}{ Swarm Intelligence (IV) } \\
\hline Algorithm Name & Acronym & Subcategory & Type & Year & Reference \\
\hline Prey Predator Algorithm & PPA & Other & Foraging & 2015 & [233] \\
\hline Particle Swarm Optimization & PSO & Flying & Movement & 1995 & [2] \\
\hline Penguins Search Optimization Algorithm & PSOA & Aquatic & Foraging & 2013 & {$[234]$} \\
\hline Regular Butterfly Optimization Algorithm & RBOA & Flying & Foraging & 2019 & {$[235]$} \\
\hline Red Deer Algorithm & RDA & Terrestrial & Movement & 2016 & [236] \\
\hline Red Fox Optimization Algorithm & RFO & Terrestrial & Foraging & 2021 & {$[237]$} \\
\hline Rhino Herd Behavior & RHB & Terrestrial & Movement & 2018 & [238] \\
\hline Roach Infestation Problem & RIO & Terrestrial & Foraging & 2008 & [239] \\
\hline Raccoon Optimization Algorithm & ROA & Terrestrial & Foraging & 2018 & {$[240]$} \\
\hline $\begin{array}{l}\text { Reincarnation } \\
\text { Algorithm }\end{array}$ & ROA.1 & Other & Movement & 2010 & [241] \\
\hline Raven Roosting Optimization Algorithm & RROA & Flying & Foraging & 2015 & [242] \\
\hline Ringed Seal Search & RSS & Aquatic & Movement & 2015 & [243] \\
\hline Shark Search Algorithm & SA & Aquatic & Foraging & 1998 & {$[244]$} \\
\hline Simulated Bee Colony & SBC & Flying & Foraging & 2009 & {$[245]$} \\
\hline Satin Bowerbird Optimizer & SBO & Flying & Movement & 2017 & [246] \\
\hline Sine Cosine Algorithm & SCA. 2 & Other & Movement & 2016 & {$[247]$} \\
\hline Snap-Drift Cuckoo Search & SDCS & Flying & Foraging & 2016 & [248] \\
\hline Shuffled Frog-Leaping Algorithm & SFLA & Terrestrial & Movement & 2006 & [249] \\
\hline Spotted Hyena Optimizer & SHO & Terrestrial & Foraging & 2017 & {$[250]$} \\
\hline Selfish Herds Optimizer & SHO.1 & Terrestrial & Movement & 2017 & {$[251]$} \\
\hline Swarm Inspired Projection Algorithm & SIP & Flying & Foraging & 2009 & {$[252]$} \\
\hline Slime Mould Algorithm & SMA & Micro & Foraging & 2008 & [253] \\
\hline Sperm Motility Algorithm & SMA.1 & Other & Movement & 2017 & {$[254]$} \\
\hline Spider Monkey Optimization & SMO & Terrestrial & Foraging & 2014 & {$[255]$} \\
\hline Seeker Optimization Algorithm & SOA & Other & Movement & 2007 & [256] \\
\hline Seagull Optimization Algorithm & SOA.1 & Flying & Foraging & 2019 & {$[257]$} \\
\hline Sandpiper Optimization Algorithm & SOA.2 & Flying & Foraging & 2020 & {$[258]$} \\
\hline Sailfish Optimizer Algorithm & SOA.3 & Aquatic & Foraging & 2019 & [259] \\
\hline Symbiosis Organisms Search & SOS & Other & Movement & 2014 & {$[260]$} \\
\hline Sooty Tern Optimization Algorithm & STOA & Flying & Movement & 2019 & {$[261]$} \\
\hline Social Spider Algorithm & SSA & Terrestrial & Foraging & 2015 & {$[262]$} \\
\hline Squirrel Search Algorithm & SSA.1 & Flying & Movement & 2019 & {$[263]$} \\
\hline Salp Swarm Algorithm & SSA.2 & Aquatic & Foraging & 2017 & [264] \\
\hline Shark Smell Optimization & SSO & Aquatic & Foraging & 2016 & {$[265]$} \\
\hline Swallow Swarm Optimization & SSO.1 & Flying & Foraging & 2013 & [266] \\
\hline Social Spider Optimization & SSO. 2 & Terrestrial & Foraging & 2013 & [267] \\
\hline Sperm Swarm Optimization Algorithm & SSOA & Other & Movement & 2018 & [268] \\
\hline See-See Partidge Chicks Optimization & SSPCO & Flying & Movement & 2015 & [269] \\
\hline $\begin{array}{lll}\text { Surface-Simplex } & \text { Swarm } & \text { Evolution } \\
\text { Algorithm } & & \end{array}$ & SSSE & Other & Movement & 2017 & {$[270]$} \\
\hline Sperm Whale Algorithm & SWA & Aquatic & Movement & 2016 & {$[271]$} \\
\hline Termite Hill Algorithm & TA & Terrestrial & Foraging & 2012 & {$[272]$} \\
\hline Termite Colony Optimization & $\mathrm{TCO}$ & Terrestrial & Foraging & 2010 & {$[273]$} \\
\hline The Great Salmon Run Algorithm & TGSR & Aquatic & Movement & 2013 & [274] \\
\hline Tunicate Swarm Algorithm & TSA.1 & Micro & Foraging & 2020 & {$[275]$} \\
\hline Virtual Ants Algorithm & VAA & Flying & Foraging & 2006 & {$[276]$} \\
\hline Virtual Bees Algorithm & VBA & Flying & Foraging & 2005 & {$[277]$} \\
\hline
\end{tabular}


Table 6: Nature- and bio-inspired meta-heuristics within the Swarm Intelligence category (IV).

\begin{tabular}{llllrr}
\hline \multicolumn{7}{c}{ Swarm Intelligence (V) } & & & \\
\hline Algorithm Name & Acronym & Subcategory & Type & Year & Reference \\
\hline Virus Colony Search & VCS & Micro & Movement & 2016 & {$[278]$} \\
Virus Optimization Algorithm & VOA.1 & Micro & Movement & 2009 & {$[279]$} \\
Viral Systems Optimization & VSO & Micro & Movement & 2008 & {$[280]$} \\
Wasp Colonies Algorithm & WCA & Flying & Foraging & 1991 & {$[10]$} \\
Wolf Colony Algorithm & WCA.1 & Terrestrial & Foraging & 2011 & {$[281]$} \\
Worm Optimization & WO & Micro & Foraging & 2014 & {$[282]$} \\
Whale Optimization Algorithm & WOA & Aquatic & Foraging & 2016 & {$[11]$} \\
Wolf Pack Search & WPS & Terrestrial & Foraging & 2007 & {$[283]$} \\
Weightless Swarm Algorithm & WSA & Other & Movement & 2012 & {$[284]$} \\
Wolf Search Algorithm & WSA.1 & Terrestrial & Foraging & 2012 & {$[285]$} \\
Wasp Swarm Optimization & WSO & Flying & Foraging & 2005 & {$[286]$} \\
Yellow Saddle Goldfish & YSGA & Aquatic & Foraging & 2018 & {$[287]$} \\
Zombie Survival Optimization & ZSO & Other & Foraging & 2012 & {$[288]$} \\
\hline
\end{tabular}

usually replicate the infection process of the cell by virus. The most known algorithm of this category is Bacterial Foraging Optimization Algorithm (BFOA, [14]).

\subsubsection{Subcategories of SI based algorithms by the inspirational behavior}

Another criterion to group SI based algorithms is the specific behavior of the animal that captured the attention of researchers and inspired the algorithm. This second criterion is also reflected in Tables 2-5, classifying each algorithm as belonging to one of the following behavioral patterns:

- Movement: We have considered that an algorithm belongs to the movement inspiration subcategory if the biological inspiration resides mainly in the way the animal inspiring the algorithm regularly moves around its environment. As such, the differential aspect of the movement could hinge on the dynamics of the movement itself (e.g. the flying movement of birds in PSO [2], jumping actions as in Shuffled Frog-Leaping Algorithm, SFLA [249], or by aquatic movements as in DPO [163]), or by the movement of the population (correspondingly, swarming movements as in Bird Swarm Algorithm, BSA [136], the migration of populations like Population Migration Algorithm, PMA [232], or the migration of particular animals like salmon [274], among others).

- Foraging: Rather than the movement strategy, in some other algorithmic variants it is the mechanism used to obtain their food what drives the behavior of the animal and, ultimately, the design of the meta-heuristic algorithm. This foraging behavior can in turn be observed in many flavors, from the tactics used by the animal at hand to surround its food source (as in the aforementioned GWO [185] and LA [202]), inspired in breeding nutrition (as Cuckoo Search [156, 289]), inspired 
in hunting techniques observed in grey wolves and lions, respectively), particular mechanisms to locate food sources and communicate its existence to the rest of the swarm (as in ACO [6]), or other exploration strategies such as the echolocation in dolphins [161], or the flashing attraction between partners observed in fireflies [4]. Sometimes, the movement of the animal also obeys to food search and retrieval. In this case, we consider that the algorithm belongs to the foraging inspiration type, rather than to the movement type. Nowadays, inspiration by foraging mechanisms is becoming more and more consolidated in the research community, appearing explicitly in the name of several bio-inspired algorithms.

\subsection{Physics/Chemistry based Algorithms}

Algorithms under this category are characterized by the fact that they imitate the behavior of physical or chemical phenomena, such as gravitational forces, electromagnetism, electric charges and water movement (in relation with physics-based approaches), and chemical reactions and gases particles movement as for chemistry-based optimization algorithms.

The complete list of reviewed algorithms in this category is provided in Tables 7 and 8 (physics-based algorithms) and Table 9 (chemistry-based methods). In this category we can find some well-known algorithms reported in the last century such as Simulated Annealing [23], or one of the most important algorithms in physics-based meta-heuristic optimization, Gravitational Search Algorithm, GSA [18]. Interestingly, a variety of space-based algorithms are rooted on GSA, such as Black Hole optimization (BH, [290]) or Galaxy Based Search Algorithm (GBSA, [19]). Other algorithms such as Harmony Search (HS, [21]) relate to the music composition process, a human invention that has more in common with other physical algorithms in what refers to the usage of sound waves than with Social Human Behavior based algorithms, the category discussed in what follows.

\subsection{Social Human Behavior based Algorithms}

Algorithms falling in this category are inspired by human social concepts, such as decision making and ideas related to the expansion/competition of ideologies inside the society as ideology (Ideology Algorithm, IA, [354]), or political concepts such as the Imperialist Colony Algorithm (ICA, [28]). This category also includes algorithms that emulate sport competitions such as the Soccer League Competition Algorithm (SLC, [24]). Brainstorming processes have also laid the inspirational foundations of several algorithms such as Brain Storm Optimization algorithm (BSO.2, [26]) and Global-Best Brain Storm Optimization algorithm (GBSO, [355]). The complete list of algorithms in this category is given in Table 10 and in Table 11.

\subsection{Plants based Algorithms}

This category essentially gathers all optimization algorithms whose search process is inspired by plants. In this case, as opposed to other methods within the Swarm Intelligence category, there is no communication between agents. One of the 
Table 7: Nature- and bio-inspired meta-heuristics within the Physics based category (I).

Physics based (I)

\begin{tabular}{|c|c|c|c|}
\hline Algorithm Name & Acronym & Year & Reference \\
\hline Artificial Electric Field Algorithm & AEFA & 2019 & [291] \\
\hline Artificial Physics Optimization & APO & 2009 & [292] \\
\hline Atom Search Optimization & ASO.1 & 2019 & [293] \\
\hline Big Bang Big Crunch & $\mathrm{BBBC}$ & 2006 & [294] \\
\hline Black Hole Optimization & $\mathrm{BH}$ & 2013 & [290] \\
\hline Colliding Bodies Optimization & $\mathrm{CBO}$ & 2014 & [295] \\
\hline Crystal Energy Optimization Algorithm & CEO & 2016 & [296] \\
\hline Central Force Optimization & $\mathrm{CFO}$ & 2008 & [297] \\
\hline Charged Systems Search & CSS & 2010 & [298] \\
\hline Electromagnetic Field Optimization & EFO & 2016 & [16] \\
\hline Electromagnetism Mechanism Optimization & EMO & 2003 & [17] \\
\hline Electimize Optimization Algorithm & EOA.1 & 2011 & [299] \\
\hline Electron Radar Search Algorithm & ERSA & 2020 & [300] \\
\hline Galaxy Based Search Algorithm & GBSA & 2011 & [19] \\
\hline Gravitational Clustering Algorithm & GCA & 1999 & [301] \\
\hline Gravitational Emulation Local Search & GELS & 2009 & [302] \\
\hline Gravitational Field Algorithm & GFA & 2010 & [303] \\
\hline Gravitational Interactions Algorithm & GIO & 2011 & [304] \\
\hline General Relativity Search Algorithm & GRSA & 2015 & [305] \\
\hline Gravitational Search Algorithm & GSA & 2009 & [18] \\
\hline Galactic Swarm Optimization & GSO.2 & 2016 & [306] \\
\hline Hydrological Cycle Algorithm & HCA & 2017 & [307] \\
\hline Harmony Elements Algorithm & HEA & 2009 & [308] \\
\hline Hysteresis for Optimization & $\mathrm{HO}$ & 2002 & [309] \\
\hline Hurricane Based Optimization Algorithm & HO. 2 & 2014 & [310] \\
\hline Harmony Search & HS & 2005 & [21] \\
\hline Intelligent Gravitational Search Algorithm & IGSA & 2012 & [311] \\
\hline Intelligence Water Drops Algorithm & IWD & 2009 & [312] \\
\hline Light Ray Optimization & LRO & 2010 & [313] \\
\hline Lightning Search Algorithm & LSA & 2015 & [314] \\
\hline Magnetic Optimization Algorithm & MFO.2 & 2008 & [315] \\
\hline Method of Musical Composition & MMC & 2014 & [316] \\
\hline Melody Search & MS.1 & 2011 & [317] \\
\hline Multi-Verse Optimizer & MVO & 2016 & [318] \\
\hline Optics Inspired Optimization & $\mathrm{OIO}$ & 2015 & [319] \\
\hline Particle Collision Algorithm & PCA & 2007 & [320] \\
\hline PopMusic Algorithm & PopMusic & 2002 & [321] \\
\hline Quantum Superposition Algorithm & QSA & 2015 & [322] \\
\hline Rain-Fall Optimization Algorithm & RFOA & 2017 & [323] \\
\hline Rain Water Algorithm & RWA & 2017 & [324] \\
\hline River Formation Dynamics & RFD & 2007 & [325] \\
\hline Radial Movement Optimization & RMO & 2014 & [326] \\
\hline Ray Optimization & RO & 2012 & [327] \\
\hline
\end{tabular}


Table 8: Nature- and bio-inspired meta-heuristics within the Physics based category (II).

Physics based (II)

\begin{tabular}{llrr}
\hline Algorithm Name & Acronym & Year & Reference \\
\hline Space Gravitational Algorithm & SGA & 2005 & {$[328]$} \\
Sonar Inspired Optimization & SIO & 2017 & {$[329]$} \\
States Matter Optimization Algorithm & SMS & 2014 & {$[330]$} \\
Spiral Dynamics Optimization & SO & 2011 & {$[331]$} \\
Spiral Optimization Algorithm & SPOA & 2010 & {$[332]$} \\
Self-Driven Particles & SPP & 1995 & {$[333]$} \\
Turbulent Flow of Water-based Optimization & TFWO & 2020 & {$[334]$} \\
Vibrating Particle Systems Algorithm & VPO & 2017 & {$[335]$} \\
Vortex Search Algorithm & VS & 2015 & {$[336]$} \\
Water Cycle Algorithm & WCA.2 & 2012 & {$[337]$} \\
Water Evaporation Optimization & WEO & 2016 & {$[338]$} \\
Water Flow-Like Algorithms & WFA & 2007 & {$[339]$} \\
Water Flow Algorithm & WFA.1 & 2007 & {$[340]$} \\
Water-Flow Algorithm Optimization & WFO & 2011 & {$[341]$} \\
Water Wave Optimization Algorithm & WWA & 2015 & {$[342]$} \\
\hline
\end{tabular}

Table 9: Nature- and bio-inspired meta-heuristics within the Chemistry based category.

Chemistry based

\begin{tabular}{llrr}
\hline Algorithm Name & Acronym & Year & Reference \\
Artificial Chemical Process & ACP & 2005 & {$[343]$} \\
Artificial Chemical Reaction Optimization Algorithm & ACROA & 2011 & {$[344]$} \\
Artificial Reaction Algorithm & ARA & 2013 & {$[345]$} \\
Chemical Reaction Optimization Algorithm & CRO.1 & 2010 & {$[346]$} \\
Gases Brownian Motion Optimization & GBMO & 2013 & {$[22]$} \\
Heat Transfer Search Algorithm & HTS & 2015 & {$[347]$} \\
Ions Motion Optimization Algorithm & IMO & 2015 & {$[348]$} \\
Integrated Radiation Optimization & IRO & 2007 & {$[349]$} \\
Kinetic Gas Molecules Optimization & KGMO & 2014 & {$[350]$} \\
Photosynthetic Algorithm & PA & 1999 & {$[351]$} \\
Simulated Annealing & SA.1 & 1989 & {$[23]$} \\
Synergistic Fibroblast Optimization & SFO & 2017 & {$[352]$} \\
Thermal Exchange Optimization & TEO & 2017 & {$[353]$} \\
\hline
\end{tabular}


Table 10: Nature- and bio-inspired meta-heuristics within the Social Human Behavior based category.

Social Human Behavior (I)

\begin{tabular}{|c|c|c|c|}
\hline Algorithm Name & Acronym & Year & Reference \\
\hline Adolescent Identity Search Algorithm & AISA & 2020 & [356] \\
\hline Anarchic Society Optimization & ASO & 2012 & [27] \\
\hline Brain Storm Optimization Algorithm & BSO.2 & 2011 & [26] \\
\hline Bus Transportation Behavior & BTA & 2019 & [357] \\
\hline Collective Decision Optimization Algorithm & CDOA & 2017 & [358] \\
\hline Cognitive Behavior Optimization Algorithm & COA.3 & 2016 & [359] \\
\hline Competitive Optimization Algorithm & COOA & 2016 & [360] \\
\hline Community of Scientist Optimization Algorithm & CSOA & 2012 & [361] \\
\hline Cultural Algorithms & CA & 1999 & [362] \\
\hline Duelist Optimization Algorithm & DOA & 2016 & [363] \\
\hline Election Algorithm & EA & 2015 & [364] \\
\hline Football Game Inspired Algorithms & FCA.1 & 2009 & [365] \\
\hline FIFA World Cup Competitions & FIFAAO & 2016 & [366] \\
\hline Golden Ball Algorithm & GBA & 2014 & [367] \\
\hline Global-Best Brain Storm Optimization Algorithm & GBSO & 2017 & [355] \\
\hline Group Counseling Optimization & GCO & 2010 & [368] \\
\hline Group Leaders Optimization Algorithm & GLOA & 2011 & [369] \\
\hline Greedy Politics Optimization Algorithm & GPO & 2014 & [370] \\
\hline Group Teaching Optimization Algorithm & GTOA & 2020 & [371] \\
\hline Human Evolutionary Model & HEM & 2007 & [372] \\
\hline Human Group Formation & HGF & 2010 & [373] \\
\hline Human-Inspired Algorithms & HIA & 2009 & [374] \\
\hline Human Urbanization Algorithm & HUA & 2020 & [375] \\
\hline Ideology Algorithm & IA & 2016 & [354] \\
\hline Imperialist Competitive Algorithm & ICA & 2007 & [28] \\
\hline Kho-Kho optimization Algorithm & KKOA & 2020 & [376] \\
\hline League Championship Algorithm & LCA.1 & 2014 & [25] \\
\hline Life Choice Based Optimizer & LCBO & 2020 & [377] \\
\hline Leaders and Followers Algorithm & LFA & 2015 & [378] \\
\hline Old Bachelor Acceptance & OBA & 1995 & [379] \\
\hline Oriented Search Algorithm & OSA & 2008 & [380] \\
\hline Parliamentary Optimization Algorithm & POA & 2008 & [381] \\
\hline Poor and Rich Optimization Algorithm & PRO & 2019 & [382] \\
\hline Queuing Search Algorithm & QSA.1 & 2018 & [383] \\
\hline Search and Rescue Algorithm & SAR & 2019 & [384] \\
\hline Social Behavior Optimization Algorithm & SBO.1 & 2003 & [385] \\
\hline Social Cognitive Optimization & SCO & 2002 & [386] \\
\hline Social Cognitive Optimization Algorithm & SCOA & 2010 & [387] \\
\hline Social Emotional Optimization Algorithm & SEA & 2010 & [388] \\
\hline Stochastic Focusing Search & SFS & 2008 & [389] \\
\hline Soccer Game Optimization & SGO & 2012 & [390] \\
\hline Soccer League Competition & SLC & 2014 & [24] \\
\hline Student Psychology Optimization Algorithm & SPBO & 2020 & [391] \\
\hline
\end{tabular}


Table 11: Nature- and bio-inspired meta-heuristics within the Social Human Behavior based category.

\begin{tabular}{llrr}
\hline \multicolumn{4}{c}{ Social Human Behavior (II) } \\
\hline Algorithm Name & Acronym & Year & Reference \\
\hline Tiki-Taka Algorithm & TTA & 2020 & {$[392]$} \\
Team Game Algorithm & TGA & 2018 & {$[393]$} \\
Teaching-Learning Based Optimization Algorithm & TLBO & 2011 & {$[394]$} \\
Thieves and Police Optimization Algorithm & TPOA & 2021 & {$[395]$} \\
Tug Of War Optimization & TWO & 2016 & {$[396]$} \\
Unconscious Search & US & 2012 & {$[397]$} \\
Volleyball Premier League Algorithm & VPL & 2017 & {$[398]$} \\
Wisdom of Artificial Crowds & WAC & 2011 & {$[399]$} \\
\hline
\end{tabular}

most well-known is Forest Optimization Algorithms (FOA.1, [400]), inspired by the process of plant reproduction. Table 12 details the specific algorithms classified in this category.

Table 12: Nature- and bio-inspired meta-heuristics within the Plants based category.

\begin{tabular}{|c|c|c|c|}
\hline \multicolumn{4}{|l|}{ Plants based } \\
\hline Algorithm Name & Acronym & Year & Reference \\
\hline Artificial Flora Optimization Algorithm & $\mathrm{AFO}$ & 2018 & [401] \\
\hline Artificial Plants Optimization Algorithm & APO.1 & 2013 & [402] \\
\hline BrunsVigia Flower Optimization Algorithm & BVOA & 2018 & [403] \\
\hline Forest Optimization Algorithm & FOA.1 & 2014 & [400] \\
\hline Flower Pollination Algorithm & FPA & 2012 & [404] \\
\hline Natural Forest Regeneration Algorithm & NFR & 2016 & [405] \\
\hline Plant Growth Optimization & PGO & 2008 & [406] \\
\hline Plant Propagation Algorithm & PPA.1 & 2009 & [407] \\
\hline Paddy Field Algorithm & PFA & 2009 & [408] \\
\hline Root Growth Optimizer & RGO & 2015 & [409] \\
\hline Root Tree Optimization Algorithm & RTOA & 2016 & [410] \\
\hline Runner Root Algorithm & RRA & 2015 & [411] \\
\hline Saplings Growing Up Algorithm & SGA.1 & 2007 & [412] \\
\hline Self-Defense Mechanism Of The Plants Algorithm & SDMA & 2018 & [413] \\
\hline Strawberry Plant Algorithm & SPA & 2014 & [414] \\
\hline Tree Growth Algorithm & TGA.1 & 2019 & [415] \\
\hline Tree Physiology Optimization & TPO & 2018 & [416] \\
\hline Tree Seed Algorithm & TSA & 2015 & [417] \\
\hline
\end{tabular}

\subsection{Algorithms with Miscellaneous Sources of Inspiration}

In this category there are include the algorithms that do not fit in any of the previous categories, i.e., we can find algorithms of diverse characteristics such as the Ying-Yang Pair Optimization (YYOP, [418]). Although this defined category is heterogeneous 
and does not exhibit any uniformity among the algorithms it represents, its inclusion in the taxonomy serves as an exemplifying fact of the very different sources of inspiration existing in the literature. The ultimate goal to reflect this miscellaneous set of algorithms is to spawn new categories once more algorithms are created by recreating similar inspirational concepts that the assorted ones already present in this category.

The complete list of algorithms in this category is in Table 13. In this regard, we stress on this pressing need for grouping assorted algorithms in years to come so as to give rise to new categories. Otherwise, if we just stockpile new algorithms without a clear correspondence to the aforementioned categories in this miscellaneous group, the overall taxonomy will not evolve and will eventually lack its main purpose: to systematically sort and ease the analysis of future advances and achievements in the field.

\section{Taxonomy by Behavior}

We now proceed with our second proposed taxonomy. In this case we sort the different algorithmic proposals reported by the community by its behavior, without any regards to their source of inspiration. To this end, a clear sorting criterion is needed that, while keeping itself agnostic with respect to its inspiration, could summarize as much as possible the different behavioral procedures characterizing the algorithms under review. The criterion adopted for this purpose is the mechanisms used for creating new solutions, or for changing existing solutions to the optimization problem. These are the main features that define the search process of each algorithm.

First, we have divided the reviewed optimization algorithms in two categories:

- Differential Vector Movement, in which new solutions are produced by a shift or a mutation performed onto a previous solution. The newly generated solution could compete against previous ones, or against other solutions in the population to achieve a space and remain therein in subsequent search iterations. This solution generation scheme implies selecting a solution as the reference, which is changed to explore the space of variables and, effectively, produce the search for the solution to the problem at hand. The most representative method of this category is arguably PSO [2], in which each solution evolves with a velocity vector to explore the search domain. Another popular algorithm with differential movement at its core is DE [53], in which new solutions are produced by adding differential vectors to existing solutions in the population. Once a solution is selected as the reference one, it is perturbed by adding the difference between other solutions. The decision as to which solutions from the population are influential in the movement is a decision that has an enormous influence on the behavior of the overall search. Consequently, we further divide this category by that decision. The movement - thus, the search - can be guided by i) all the population (Figure 4.a); ii) only the significant/relevant solutions, e.g., the best and/or the worst candidates in the population (Figure 4.b); or iii) a small group, which could stand 
Table 13: Nature- and bio-inspired meta-heuristics within the Miscellaneous category.

\begin{tabular}{|c|c|c|c|}
\hline \multicolumn{4}{|c|}{ Miscellaneous } \\
\hline Algorithm Name & Acronym & Year & Reference \\
\hline Atmosphere Clouds Model & $\mathrm{ACM}$ & 2013 & [419] \\
\hline Artificial Cooperative Search & ACS & 2012 & [420] \\
\hline Innovative Gunner Algorithm & AIG & 2019 & [421] \\
\hline Across Neighbourhood Search & ANS & 2016 & [422] \\
\hline Battle Royale Optimization Algorithm & $\mathrm{BRO}$ & 2020 & [423] \\
\hline Bar Systems & BS.2 & 2008 & [424] \\
\hline Backtracking Search Optimization & BSO. 3 & 2012 & [425] \\
\hline Cloud Model-Based Algorithm & CMBDE & 2012 & [426] \\
\hline Chaos Optimization Algorithm & COA.4 & 1998 & [427] \\
\hline Clonal Selection Algorithm & CSA. 1 & 2000 & [428] \\
\hline COVID-19 Optimizer Algorithm & CVA & 2020 & [429] \\
\hline Dice Game Optimizer & DGO & 2019 & [430] \\
\hline Dialectic Search & DS & 2009 & [431] \\
\hline Differential Search Algorithm & DSA & 2012 & [432] \\
\hline Exchange Market Algorithm & EMA & 2014 & [433] \\
\hline Extremal Optimization & EO & 2000 & [434] \\
\hline Fireworks Algorithm Optimization & FAO & 2010 & [435] \\
\hline Farmland Fertility Algorithm & FFA & 2018 & [436] \\
\hline Grenade Explosion Method & GEM & 2010 & [437] \\
\hline Golden Sine Algorithm & GSA.1 & 2017 & [438] \\
\hline Heart Optimization & HO.1 & 2014 & [439] \\
\hline Hyper-parameter Dialectic Search & HDS & 2020 & [440] \\
\hline Ideological Sublations & IS & 2017 & [441] \\
\hline Interior Search Algorithm & ISA & 2014 & [442] \\
\hline Keshtel Algorithm & KA & 2014 & [443] \\
\hline Kidney-Inspired Algorithm & KA.1 & 2017 & [444] \\
\hline Kaizen Programming & $\mathrm{KP}$ & 2014 & [445] \\
\hline Membrane Algorithms & MA & 2005 & [446] \\
\hline Mine Blast Algorithm & MBA & 2013 & [447] \\
\hline Neuronal Communication Algorithm & NCA & 2017 & [448] \\
\hline Pearl Hunting Algorithm & PHA & 2012 & [449] \\
\hline Passing Vehicle Search & PVS & 2016 & [450] \\
\hline Artificial Raindrop Algorithm & RDA.1 & 2014 & [15] \\
\hline Reactive Dialectic Search & RDS & 2017 & [451] \\
\hline Scientifics Algorithms & SA.2 & 2014 & {$[452]$} \\
\hline Social Engineering Optimization & SEO & 2017 & [453] \\
\hline Stochastic Fractal Search & SFS. 1 & 2015 & {$[454]$} \\
\hline Search Group Algorithm & SGA.2 & 2015 & {$[455]$} \\
\hline Simple Optimization & SOPT & 2012 & [456] \\
\hline Small World Optimization & SWO & 2006 & [457] \\
\hline The Great Deluge Algorithm & TGD & 1993 & {$[458]$} \\
\hline & WDO & 2010 & [459] \\
\hline Ying-Yang Pair Optimization & YYOP & 2016 & [418] \\
\hline
\end{tabular}


for the neighborhood around each solution or, in algorithms with subpopulations, only the subpopulation to which each solution belongs (Figure 4.c).

- Solution creation, in which new solutions are not generated by mutation/movement of a single reference solution, but instead by combining several solutions (so there is not only a single parent solution), or other similar mechanism. Two approaches can be utilized for creating new solutions. The first one is by combination, or crossover of several solutions (Figure 4.d). The classical GA [86] is the most straightforward example of this type. Another approach is by stigmergy (Figure 4.e), in which there is an indirect coordination between the different solutions or agents, usually using an intermediate structure, to generate better ones. A classical example of stigmergy for creating solutions is ACO [7], in which new solutions are generated by the trace of pheromones left by different agents on a graph representing the solution space of the problem under analysis.

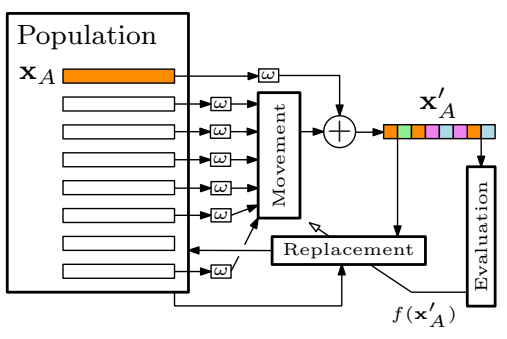

(a)

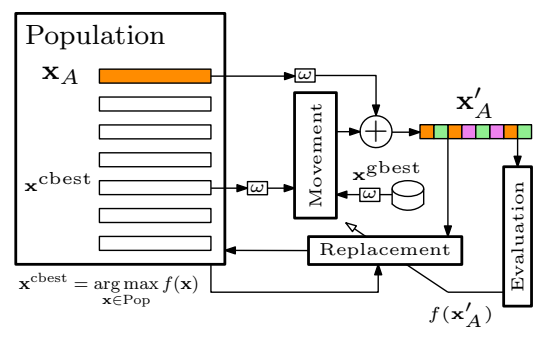

(b)

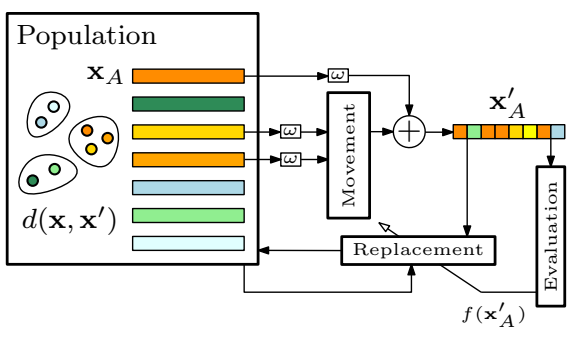

(c)

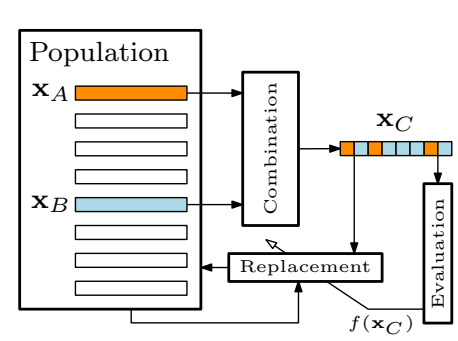

(d)

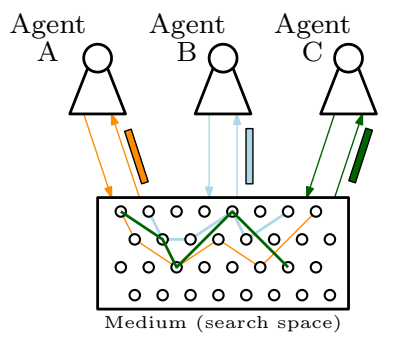

(e)

Figure 4: Schematic diagrams of the different algorithmic behaviors on which our second taxonomy relies. The upper plots illustrate the process of generating new solutions by Differential Vector Movement from a given solution $\mathbf{x}_{A}$, using (a) the entire population; (b) relevant individuals (in the example, the movement results from a weighted combination - $\omega-$ of the current best solution in the population and the best solution found so far by the algorithm); and (c) neighboring solutions in the population to the reference individual. The lower plots show the same process using solution creation by (d) combination; and (e) stigmergy.

Bearing the above criteria in mind, Figure 5 shows the classification reached after our literature analysis. The plot indicates, for the 412 reviewed algorithms, the number and ratio of proposals classified in each category and subcategory. It can be observed that in most nature- and bio-inspired algorithms, new solutions are generated by differential vector movement over 
existing ones (66\% vs 34\%). Among them, the search process is mainly guided by representative solutions (near 55\% in global, almost $83 \%$ from this category), mainly the so-called current best solution (in a very similar fashion to the naive version of the PSO solver). Thus, the creation of new solutions by movement vectors oriented towards the best solution is the search mechanism found in more than half (55\%) of all the 412 reviewed proposals.

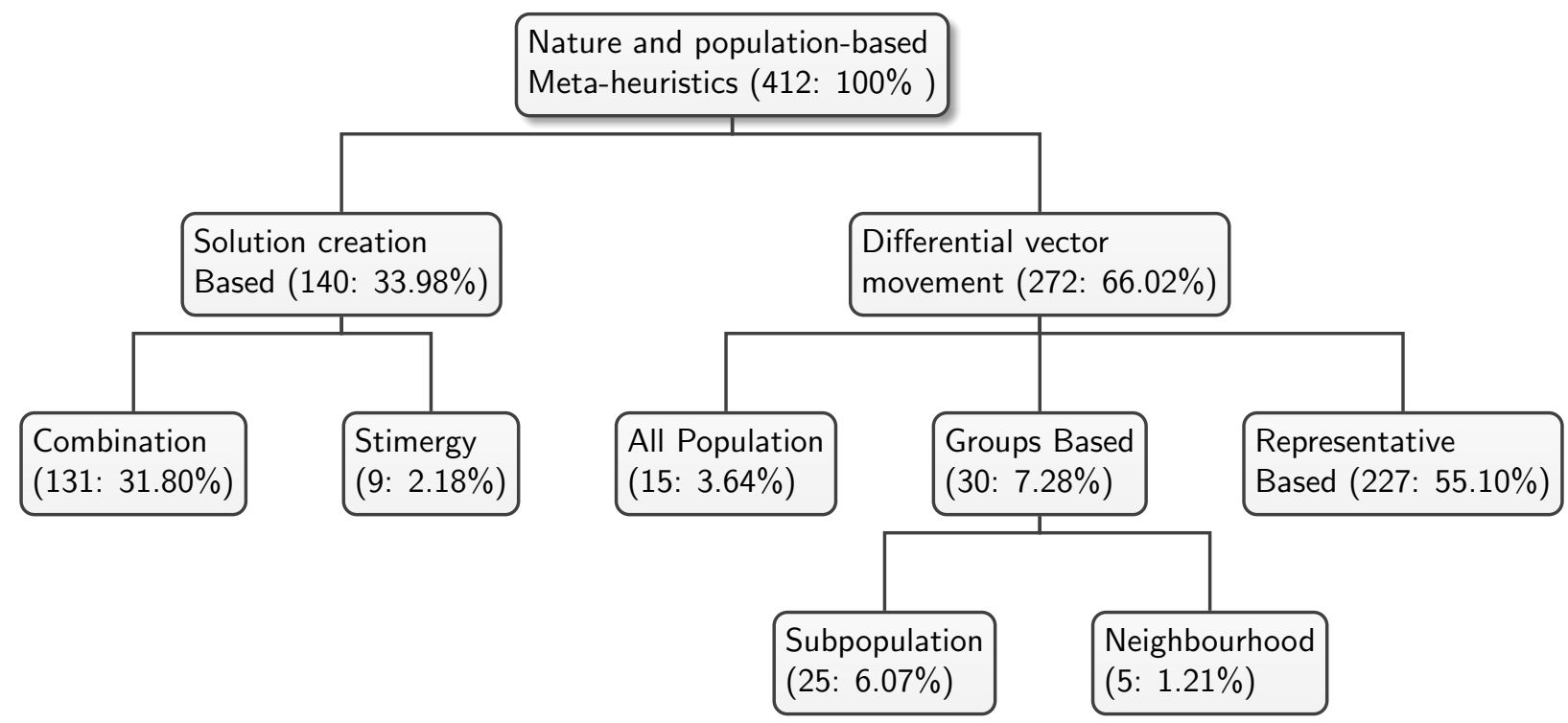

Figure 5: Classification of the reviewed papers using the behavior taxonomy.

The following subsections provide a brief global view of the different categories introduced above. For each category we describe its main characteristics, an example, and a table with the algorithms belonging to that category.

\subsection{Differential Vector Movement}

This category of our behavior-based taxonomy amounts up to $66 \%$ of the analyzed algorithms. In all of them, new solutions are obtained by a movement departing from existing solutions. By using a solution as the reference, a differential vector is used to move from the reference towards a new candidate, that could replace the previous one or instead compete to be included into the population.

The crucial decision in differential vector movement is how the differential vector (namely, the intensity and direction of the movement) is calculated. This differential vector could be calculated so as to move the reference solution to another solution (usually a better one), or as a lineal combination of other different solutions, allowing the combination of attraction vectors (toward the best solutions) with repulsion vectors (away from worse ones, or from other solutions, to enforce diversity). The mathematical nature of this operation usually restricts the domain of the representation to a numerical, usually real-valued representation. 
This category is further divided into subcategories as a function of the above decision, i.e. which solutions are considered to create the movement vector. It should be noted that some algorithms can be classified into more than one subcategory. For instance, a particle's update in the PSO solver is affected by the global best particle behavior and certain local best particle(s) behavior. The local best behavior can be either dependent on the particle's previous behavior or the behavior of some particles in its neighborhood. This makes PSO a possible member of two of the subcategories, namely, Differential Vector as a Function of Representative Solutions and Differential Vector as a Function of a Group of Solutions. Nevertheless, we have considered the classical PSO as a member of Representative Solutions because the influence of the best algorithm is stronger than the influence of the neighborhood. In any case, following the above rationale, other PSO variants could fall within any other subcategory. We now describe each of such subcategories.

\subsubsection{Differential Vector as a Function of the Entire Population}

One possible criterion is used all the individuals in the population to generate the movement of each solution. In these algorithms, all individuals have a degree of influence on the movement of the other solutions. Such a degree is usually weighted according to the fitness difference and/or distance between solutions. A significant example is FA [4], in which a solution suffers a moving force towards better solutions as a function of their distance. Consequently, solutions closer to the reference solution will have a stronger influence than more distant counterparts. As shown in Table 14, algorithms in this subcategory belong to different categories in the previous inspiration source based taxonomy.

Table 14: Nature- and bio-inspired meta-heuristics within the Differential Vector Movement category, wherein the differential vector is influenced by the entire population.

Influenced by the entire population

\begin{tabular}{llrr}
\hline Algorithm Name & Acronym & Year & Reference \\
\hline Artificial Electric Field Algorithm & AEFA & 2019 & {$[291]$} \\
Artificial Plants Optimization Algorithm & APO.1 & 2013 & {$[402]$} \\
Chaotic Dragonfly Algorithm & CDA & 2018 & {$[149]$} \\
Central Force Optimization & CFO & 2008 & {$[297]$} \\
Charged Systems Search & CSS & 2010 & {$[298]$} \\
Electromagnetism Mechanism Optimization & EMO & 2003 & {$[17]$} \\
Firefly Algorithm & FA & 2009 & {$[4]$} \\
Gravitational Clustering Algorithm & GCA & 1999 & {$[301]$} \\
Group Counseling Optimization & GCO & 2010 & {$[368]$} \\
Gravitational Search Algorithm & GSA & 2009 & {$[18]$} \\
Human Group Formation & HGF & 2010 & {$[373]$} \\
Hoopoe Heuristic Optimization & HHO.1 & 2012 & {$[189]$} \\
Intelligent Gravitational Search Algorithm & IGSA & 2012 & {$[311]$} \\
Integrated Radiation Optimization & IRO & 2007 & {$[349]$} \\
Locust Swarms Search & LSS & 2015 & {$[208]$} \\
\hline
\end{tabular}




\subsubsection{Differential Vector as a Function of Representative Solutions}

In this group (the most populated in this second taxonomy), the different movement of each solution is only influenced by a small group of representative solutions. It is often the case that these representative solutions are selected to be the best solutions found by the algorithm (as per the objective of the problem at hand), being able to be guided only by e.g. the current best individual of the population.

Tables 15, 16, 17, 18 and 19 show the different algorithms in this subcategory. An exemplary algorithm of this category that has been a major meta-heuristic solver in the history of the field is PSO [2]. In this solver, each solution or particle is guided by the global current best solution and the best solution obtained by that particle during the search. Another classical algorithm in this category is the majority of the family of DE approaches [53]. In most of the variants of this evolutionary algorithm, the influence of the best solution(s) is hybridized with a differential vector that perturbs the new solution toward random individuals for the sake of an increased diversity along the search. However, this subcategory also includes many other algorithms with differences as considering nearly better solutions (as in the Bat Inspired Algorithm [3] or the Brain Storm Optimization Algorithm [26]) or the worse solutions (to avoid less promising regions), as in the Grasshopper Optimization Algorithm (GOA, [5]). More than half of all algorithmic proposals dwell into this subcategory, with a prominence of Swarm Intelligence solvers due to their behavioral inspiration in PSO and DE. We will revolve on these identified similarities in Section 5.

\subsubsection{Differential Vector as a Function of a Group of Solutions}

Algorithms within this category do not resort to representative solutions of the entire population (such as the current best), but they only consider solutions of a subset or group of the solutions in the population. When the differential movement considers both a group and a representative of all the population, the algorithm under analysis is considered to belong to the previous subcategory, because the representative has usually the strongest influence over the search. Two different subcategories hold when a group of solutions is used for computing the differential movement vector:

- Subpopulation based differential vector: In algorithms belonging to this subcategory (listed in Table 20) the population is divided in several subpopulations, such that the movement of each solution is only affected by the other solutions in the same subpopulation. Examples of algorithms in this subcategory are LA [202] or the Monarch Butterfly Optimization algorithm (MBO, [211]).

- Neighborhood based differential vector: In this subcategory, each solution is affected only by solutions in its local neighborhood. Table 21 compiles all algorithms that are classified in this subcategory. A notable example in this list is BFOA [14], in which all solutions in the neighborhood impact on the computation of the movement vector, either by 
Table 15: Nature- and bio-inspired meta-heuristics within the Differential Vector Movement category, wherein the differential vector is influenced by representative solutions (I).

\begin{tabular}{|c|c|c|c|}
\hline \multicolumn{4}{|c|}{ Influenced by representative solutions (I) } \\
\hline Algorithm Name & Acronym & Year & Reference \\
\hline Artificial Algae Algorithm & AAA & 2015 & [103] \\
\hline Artificial Bee Colony & $\mathrm{ABC}$ & 2007 & [8] \\
\hline Animal Behavior Hunting & $\mathrm{ABH}$ & 2014 & [105] \\
\hline African Buffalo Optimization & $\mathrm{ABO}$ & 2016 & [106] \\
\hline Atmosphere Clouds Model & $\mathrm{ACM}$ & 2013 & [419] \\
\hline Artificial Feeding Birds & AFB & 2018 & [108] \\
\hline Adolescent Identity Search Algorithm & AISA & 2020 & [356] \\
\hline Ant Lion Optimizer & ALO & 2015 & [110] \\
\hline Animal Migration Optimization & AMO & 2014 & [109] \\
\hline Across Neighbourhood Search & ANS & 2016 & [422] \\
\hline Anarchic Society Optimization & ASO & 2012 & [27] \\
\hline Atom Search Optimization & ASO.1 & 2019 & [293] \\
\hline Artificial Searching Swarm Algorithm & ASSA & 2009 & [112] \\
\hline Artificial Tribe Algorithm & ATA & 2009 & [113] \\
\hline African Wild Dog Algorithm & AWDA & 2013 & [114] \\
\hline Bison Behavior Algorithm & BBA & 2019 & [118] \\
\hline Big Bang Big Crunch & $\mathrm{BBBC}$ & 2006 & [294] \\
\hline Bacterial Chemotaxis Optimization & BCO.2 & 2002 & [122] \\
\hline Bacterial Colony Optimization & BCO.1 & 2012 & [121] \\
\hline Border Collie Optimization & BCO. 3 & 2020 & [123] \\
\hline Bald Eagle Search Optimization & BES & 2019 & [115] \\
\hline Black Hole Optimization & BH & 2013 & [290] \\
\hline Bat Intelligence & BI & 2012 & [127] \\
\hline Bat Inspired Algorithm & BIA & 2010 & [3] \\
\hline Biology Migration Algorithm & BMA & 2019 & [128] \\
\hline Blind, Naked Mole-Rats Algorithm & BNMR & 2013 & [130] \\
\hline Butterfly Optimizer & BO & 2015 & [131] \\
\hline Bonobo Optimizer & BO.1 & 2019 & [132] \\
\hline Battle Royale Optimization Algorithm & $\mathrm{BRO}$ & 2020 & [423] \\
\hline Bird Swarm Algorithm & BSA & 2016 & [136] \\
\hline Bee Swarm Optimization & $\mathrm{BSO}$ & 2010 & [137] \\
\hline Bioluminiscent Swarm Optimization Algorithm & BSO.1 & 2011 & [138] \\
\hline Brain Storm Optimization Algorithm & BSO.2 & 2011 & [26] \\
\hline Buzzard Optimization Algorithm & BUZOA & 2019 & [140] \\
\hline Binary Whale Optimization Algorithm & BWOA & 2019 & [142] \\
\hline Collective Animal Behavior & $\mathrm{CAB}$ & 2012 & [143] \\
\hline Catfish Optimization Algorithm & $\mathrm{CAO}$ & 2011 & [145] \\
\hline Cheetah Based Algorithm & CBA & 2018 & [144] \\
\hline Cricket Behavior-Based Algorithm & CBBE & 2016 & [146] \\
\hline Chaotic Crow Search Algorithm & CCSA & 2018 & [148] \\
\hline Collective Decision Optimization Algorithm & CDOA & 2017 & [358] \\
\hline Camel Herd Algorithm & CHA & 2017 & [152] \\
\hline Cloud Model-Based Algorithm & CMBDE & 2012 & [426] \\
\hline Camel Traveling Behavior & COA.1 & 2016 & [154] \\
\hline Coyote Optimization Algorithm & COA.2 & 2018 & [155] \\
\hline Cognitive Behavior Optimization Algorithm & COA.3 & 2016 & [359] \\
\hline Chaos Optimization Algorithm & COA.4 & 1998 & [427] \\
\hline
\end{tabular}


Table 16: Nature- and bio-inspired meta-heuristics within the Differential Vector Movement category, wherein the differential vector is influenced by representative solutions (II).

\begin{tabular}{|c|c|c|c|}
\hline \multicolumn{4}{|c|}{ Influenced by representative solutions (II) } \\
\hline Algorithm Name & Acronym & Year & Reference \\
\hline Competitive Optimization Algorithm & COOA & 2016 & [360] \\
\hline Crow Search Algorithm & CSA & 2016 & [157] \\
\hline Chameleon Swarm Algorithm & CSA. 2 & 2021 & [73] \\
\hline Cat Swarm Optimization & $\mathrm{CSO}$ & 2006 & [158] \\
\hline Community of Scientist Optimization Algorithm & CSOA & 2012 & [361] \\
\hline Dragonfly Algorithm & DA & 2016 & [9] \\
\hline Differential Evolution & DE & 1997 & [81] \\
\hline Deer Hunting Optimization Algorithm & DHOA & 2019 & [162] \\
\hline Dolphin Partner Optimization & DPO & 2009 & [163] \\
\hline Differential Search Algorithm & DSA & 2012 & [432] \\
\hline Donkey Theorem Optimization & DTO & 2019 & [164] \\
\hline Elephant Herding Optimization & EHO & 2016 & [165] \\
\hline Emperor Penguin Optimizer & EPO & 2018 & [167] \\
\hline Electron Radar Search Algorithm & ERSA & 2020 & [300] \\
\hline Elephant Search Algorithm & ESA & 2015 & [169] \\
\hline Eagle Strategy & ES.1 & 2010 & [168] \\
\hline Elephant Swarm Water Search Algorithm & ESWSA & 2018 & [170] \\
\hline Fireworks Algorithm Optimization & FAO & 2010 & [435] \\
\hline Flocking Base Algorithms & FBA & 2006 & [172] \\
\hline Fast Bacterial Swarming Algorithm & FBSA & 2008 & [173] \\
\hline Football Game Inspired Algorithms & FCA.1 & 2009 & [365] \\
\hline Farmland Fertility Algorithm & FFA & 2018 & [436] \\
\hline FIFA World Cup Competitions & FIFAAO & 2016 & [366] \\
\hline Flock by Leader & FL & 2012 & [175] \\
\hline Fruit Fly Optimization Algorithm & FOA & 2012 & [176] \\
\hline Falcon Optimization Algorithm & FOA.2 & 2019 & [177] \\
\hline Flower Pollination Algorithm & FPA & 2012 & [404] \\
\hline Fish-Swarm Algorithm & FSA & 2002 & [178] \\
\hline Fish Swarm Algorithm & FSA.1 & 2011 & [179] \\
\hline Fish School Search & FSS & 200 & [180] \\
\hline Gases Brownian Motion Optimization & GBMO & 2013 & [22] \\
\hline Global-Best Brain Storm Optim & GBSO & 201 & [355] \\
\hline Group Escape Behavior & GEB & 2011 & [181] \\
\hline Grenade Explosion Method & GEM & 2010 & [437] \\
\hline Gravitational Field Algorithm & GFA & 2010 & [303] \\
\hline Gravitational Interactions Algorithm & GIO & 2011 & [304] \\
\hline Good Lattice Swarm Optimization & GLSO & 2007 & [182] \\
\hline Grasshopper Optimisation Algorithm & GOA & 2017 & [5] \\
\hline General Relativity Search Algorithm & GRSA & 2015 & [305] \\
\hline Golden Sine Algorithm & GSA.1 & 2017 & [438] \\
\hline Glowworm Swarm Optimization & GSO & 2013 & [20] \\
\hline Galactic Swarm Optimization & GSO.2 & 2016 & [306] \\
\hline Goose Team Optimization & GTO & 2008 & [184] \\
\hline Group Teaching Optimization Algorithm & GTOA & 2020 & [371] \\
\hline Grey Wolf Optimizer & GWO & 2014 & [185] \\
\hline Hitchcock Birds-Inspired Algorithm & HBIA & 2020 & [186] \\
\hline Hydrological Cycle Algorithm & HCA & 2017 & [307] \\
\hline
\end{tabular}


Table 17: Nature- and bio-inspired meta-heuristics within the Differential Vector Movement category, wherein the differential vector is influenced by representative solutions (III).

\begin{tabular}{|c|c|c|c|}
\hline \multicolumn{4}{|c|}{ Influenced by representative solutions (III) } \\
\hline Algorithm Name & Acronym & Year & Reference \\
\hline Harry's Hawk Optimization Algorithm & $\mathrm{HHO}$ & 2019 & [188] \\
\hline Heart Optimization & HO.1 & 2014 & [439] \\
\hline Hurricane Based Optimization Algorithm & HO.2 & 2014 & [310] \\
\hline Hybrid Rice Optimization & $\mathrm{HRO}$ & 2016 & [88] \\
\hline Hunting Search & $\mathrm{HuS}$ & 2010 & [191] \\
\hline Honeybee Social Foraging & HSF & 2007 & [192] \\
\hline Heat Transfer Search Algorithm & HTS & 2015 & [347] \\
\hline Human Urbanization Algorithm & HUA & 2020 & [375] \\
\hline Ideology Algorithm & IA & 2016 & [354] \\
\hline Imperialist Competitive Algorithm & ICA & 2007 & [28] \\
\hline Ideological Sublations & IS & 2017 & [441] \\
\hline Interior Search Algorithm & ISA & 2014 & [442] \\
\hline Jaguar Algorithm & JA & 2015 & [198] \\
\hline Kidney-Inspired Algorithm & KA.1 & 2017 & [444] \\
\hline Kinetic Gas Molecules Optimization & KGMO & 2014 & {$[350]$} \\
\hline Krill Herd & $\mathrm{KH}$ & 2012 & [13] \\
\hline Kho-Kho optimization Algorithm & KKOA & 2020 & [376] \\
\hline Krestrel Search Algorithm & KSA & 2016 & [200] \\
\hline Killer Whale Algorithm & KWA & 2017 & [201] \\
\hline Seven-Spot Ladybird Optimization & LBO & 2013 & [203] \\
\hline League Championship Algorithm & LCA.1 & 2014 & [25] \\
\hline Leaders and Followers Algorithm & LFA & 2015 & [378] \\
\hline Lightning Search Algorithm & LSA & 2015 & [314] \\
\hline Locust Swarms Optimization & LSO & 2009 & [207] \\
\hline Membrane Algorithms & MA & 2005 & [446] \\
\hline Mayfly Optimization Algorithm & MA.1 & 2020 & [209] \\
\hline Mine Blast Algorithm & MBA & 2013 & [447] \\
\hline Magnetotactic Bacteria Optimization Algorithm & $\mathrm{MBO}$ & 2013 & [210] \\
\hline Mouth Breeding Fish Algorithm & MBF & 2018 & [213] \\
\hline Modified Cuckoo Search & MCS & 2009 & [214] \\
\hline Modified Cockroach Swarm Optimization & MCSO & 2011 & [215] \\
\hline Moth Flame Optimization Algorithm & MFO & 2015 & [216] \\
\hline Magnetic Optimization Algorithm & MFO.2 & 2008 & [315] \\
\hline Meerkats Inspired Algorithm & MIA & 2018 & [218] \\
\hline Marine Predators Algorithm & MPA & 2020 & {$[220]$} \\
\hline Mushroom Reproduction Optimization & MRO & 2018 & [93] \\
\hline Monkey Search & MS & 2007 & [221] \\
\hline Multi-Verse Optimizer & MVO & 2016 & [318] \\
\hline Naked Moled Rat & NMR & 2019 & [223] \\
\hline Nomadic People Optimizer & NPO & 2019 & [224] \\
\hline Orcas Intelligence Algorithm & $\mathrm{OA}$ & 2020 & [225] \\
\hline OptBees & OB & 2012 & [226] \\
\hline Optimal Foraging Algorithm & OFA & 2017 & [227] \\
\hline Optics Inspired Optimization & $\mathrm{OIO}$ & 2015 & [319] \\
\hline Owls Optimization Algorithm & OOA & 2019 & [228] \\
\hline Oriented Search Algorithm & OSA & 2008 & [380] \\
\hline Paddy Field Algorithm & PFA & 2009 & [408] \\
\hline
\end{tabular}


Table 18: Nature- and bio-inspired meta-heuristics within the Differential Vector Movement category, wherein the differential vector is influenced by representative solutions (IV).

\begin{tabular}{|c|c|c|c|}
\hline \multicolumn{4}{|c|}{ Influenced by representative solutions (IV) } \\
\hline Algorithm Name & Acronym & Year & Reference \\
\hline Pigeon Inspired Optimization & PIO & 2014 & [231] \\
\hline Population Migration Algorithm & PMA & 2009 & [232] \\
\hline Parliamentary Optimization Algorithm & POA & 2008 & [381] \\
\hline Prey Predator Algorithm & PPA & 2015 & [233] \\
\hline Plant Propagation Algorithm & PPA.1 & 2009 & [407] \\
\hline Poor and Rich Optimization Algorithm & PRO & 2019 & [382] \\
\hline Particle Swarm Optimization & PSO & 1995 & [2] \\
\hline Penguins Search Optimization Algorithm & PSOA & 2013 & [234] \\
\hline Passing Vehicle Search & PVS & 2016 & [450] \\
\hline Queuing Search Algorithm & QSA.1 & 2018 & [383] \\
\hline Regular Butterfly Optimization Algorithm & RBOA & 2019 & [235] \\
\hline Artificial Raindrop Algorithm & RDA.1 & 2014 & [15] \\
\hline Red Fox Optimization Algorithm & RFO & 2021 & [237] \\
\hline Root Growth Optimizer & RGO & 2015 & [409] \\
\hline Roach Infestation Problem & RIO & 2008 & [239] \\
\hline Radial Movement Optimization & RMO & 2014 & [326] \\
\hline Ray Optimization & RO & 2012 & [327] \\
\hline Runner Root Algorithm & RRA & 2015 & [411] \\
\hline Raven Roosting Optimization Algorithm & RROA & 2015 & [242] \\
\hline Root Tree Optimization Algorithm & RTOA & 2016 & [410] \\
\hline Rain Water Algorithm & RWA & 2017 & [324] \\
\hline Search and Rescue Algorithm & SAR & 2019 & [384] \\
\hline Satin Bowerbird Optimizer & SBO & 2017 & [246] \\
\hline Stem Cells Algorithm & SCA & 2011 & [96] \\
\hline Sine Cosine Algorithm & SCA. 2 & 2016 & [247] \\
\hline Social Cognitive Optimization & $\mathrm{SCO}$ & 2002 & [386] \\
\hline Social Cognitive Optimization Algorithm & SCOA & 2010 & [387] \\
\hline Social Emotional Optimization Algorithm & SEA & 2010 & [388] \\
\hline Synergistic Fibroblast Optimization & SFO & 2017 & [352] \\
\hline Stochastic Focusing Search & SFS & 2008 & [389] \\
\hline Stochastic Fractal Search & SFS.1 & 2015 & [454] \\
\hline Space Gravitational Algorithm & SGA & 2005 & [328] \\
\hline Soccer Game Optimization & SGO & 2012 & [390] \\
\hline Spotted Hyena Optimizer & SHO & 2017 & [250] \\
\hline Selfish Herds Optimizer & SHO.1 & 2017 & [251] \\
\hline Swarm Inspired Projection Algorithm & SIP & 2009 & [252] \\
\hline Soccer League Competition & SLC & 2014 & [24] \\
\hline Slime Mould Algorithm & SMA & 2008 & [253] \\
\hline Sperm Motility Algorithm & SMA.1 & 2017 & [254] \\
\hline Spider Monkey Optimization & SMO & 2014 & [255] \\
\hline States Matter Optimization Algorithm & SMS & 2014 & [330] \\
\hline Spiral Dynamics Optimization & SO & 2011 & [331] \\
\hline Student Psychology Optimization Algorithm & SPBO & 2020 & [391] \\
\hline Spiral Optimization Algorithm & SPOA & 2010 & [332] \\
\hline Self-Driven Particles & SPP & 1995 & [333] \\
\hline Seeker Optimization Algorithm & SOA & 2007 & [256] \\
\hline Seagull Optimization Algorithm & SOA.1 & 2019 & [257] \\
\hline
\end{tabular}


Table 19: Nature- and bio-inspired meta-heuristics within the Differential Vector Movement category, wherein the differential vector is influenced by representative solutions $(\mathrm{V})$.

\begin{tabular}{|c|c|c|c|}
\hline \multicolumn{4}{|c|}{ Influenced by representative solutions (V) } \\
\hline Algorithm Name & Acronym & Year & Reference \\
\hline Sandpiper Optimization Algorithm & SOA.2 & 2020 & [258] \\
\hline Sailfish Optimizer Algorithm & SOA.3 & 2019 & [259] \\
\hline Symbiosis Organisms Search & SOS & 2014 & [260] \\
\hline Sooty Tern Optimization Algorithm & STOA & 2019 & [261] \\
\hline Social Spider Algorithm & SSA & 2015 & [262] \\
\hline Squirrel Search Algorithm & SSA.1 & 2019 & [263] \\
\hline Shark Smell Optimization & SSO & 2016 & [265] \\
\hline Swallow Swarm Optimization & SSO.1 & 2013 & [266] \\
\hline Social Spider Optimization & SSO. 2 & 2013 & [267] \\
\hline Sperm Swarm Optimization Algorithm & SSOA & 2018 & [268] \\
\hline See-See Partridge Chicks Optimization & SSPCO & 2015 & [269] \\
\hline Surface-Simplex Swarm Evolution Algorithm & SSSE & 2017 & [270] \\
\hline Termite Colony Optimization & TCO & 2010 & [273] \\
\hline Team Game Algorithm & TGA & 2018 & [393] \\
\hline The Great Salmon & TGSR & 2013 & [274] \\
\hline Teaching-Leaning Based Optimization Algorithm & TLBO & 2011 & [394] \\
\hline Tree Physiology Optimization & TPO & 2018 & [416] \\
\hline Thieves and Police Optimization Algorithm & TPOA & 2021 & [395] \\
\hline Tree Seed Algorithm & TSA & 2015 & [417] \\
\hline Tunicate Swarm Algorithm & TSA.1 & 2020 & [275] \\
\hline Tiki-Taka Algorithm & TTA & 2020 & [392] \\
\hline Tug Of War Optimization & TWO & 2016 & [396] \\
\hline Unconscious Search & US & 2012 & [397] \\
\hline Virus Colony Search & VCS & 2016 & [278] \\
\hline Variable Mesh Optimization & VMO & 2012 & [100] \\
\hline Volleyball Premier League Algorithm & VPL & 2017 & [398] \\
\hline Vibrating Particle Systems Algorithm & VPO & 2017 & [335] \\
\hline Vortex Search Algorithm & VS & 2015 & [336] \\
\hline Wolf Colony Algorithm & WCA.1 & 2011 & [281] \\
\hline Water Cycle Algorithm & WCA.2 & 2012 & [337] \\
\hline Wind Driven Optimization & WDO & 2010 & [459] \\
\hline Water Evaporation Optimization & WEO & 2016 & [338] \\
\hline Whale Optimization Algorithm & WOA & 2016 & [11] \\
\hline Wolf Pack Search & WPS & 2007 & [283] \\
\hline Weightless Swarm Algorithm & WSA & 2012 & [284] \\
\hline Wolf Search Algorithm & WSA.1 & 2012 & [285] \\
\hline Water Wave Optimization Algorithm & WWA & 2015 & [342] \\
\hline Yellow Saddle Goldfish & YSGA & 2018 & [287] \\
\hline Zombie Survival Optimization & ZSO & 2012 & [288] \\
\hline
\end{tabular}


Table 20: Nature- and bio-inspired meta-heuristics within the Differential Vector Movement category, wherein the differential vector is influenced by subpopulations.

\section{Influenced by subpopulations}

\begin{tabular}{lllr}
\hline Algorithm Name & Acronym & Year & Reference \\
\hline Artificial Chemical Process & ACP & 2005 & {$[343]$} \\
Artificial Cooperative Search & ACS & 2012 & {$[420]$} \\
Artificial Physics Optimization & APO & 2009 & {$[292]$} \\
Bee Colony-Inspired Algorithm & BCIA & 2009 & {$[119]$} \\
Colliding Bodies Optimization & CBO & 2014 & {$[295]$} \\
Cuttlefish Algorithm & CFA & 2013 & {$[150]$} \\
Cuckoo Optimization Algorithm & COA & 2011 & {$[153]$} \\
Chicken Swarm Optimization & CSO.1 & 2014 & {$[159]$} \\
COVID-19 Optimizer Algorithm & CVA & 2020 & {$[429]$} \\
Dice Game Optimizer & DGO & 2019 & {$[430]$} \\
Exchange Market Algorithm & EMA & 2014 & {$[433]$} \\
Greedy Politics Optimization Algorithm & GPO & 2014 & {$[370]$} \\
Group Search Optimizer & GSO.1 & 2009 & {$[183]$} \\
Horse Optimization Algorithm & HOA & 2020 & {$[190]$} \\
Hierarchical Swarm Model & HSM & 2010 & {$[193]$} \\
Ions Motion Optimization Algorithm & IMO & 2015 & {$[348]$} \\
Life Choice Based Optimizer & LCBO & 2020 & {$[377]$} \\
Lion Optimization Algorithm & LOA & 2016 & {$[205]$} \\
Monarch Butterfly Optimization & MBO.1 & 2017 & {$[211]$} \\
Social Behavior Optimization Algorithm & SBO.1 & 2003 & {$[385]$} \\
Sperm Whale Algorithm & SWA & 2016 & {$[271]$} \\
Thermal Exchange Optimization & TEO & 2017 & {$[353]$} \\
Turbulent Flow of Water-based Optimization & TFWO & 2020 & {$[334]$} \\
Wisdom of Artificial Crowds & WAC & 2011 & {$[399]$} \\
Worm Optimization & WO & 2014 & {$[282]$} \\
\hline
\end{tabular}


attracting the solution (if the neighboring solution has better fitness than the reference solution) or in a repulsive way (when the neighboring solution is worse than the one to be moved).

Table 21: Nature- and bio-inspired meta-heuristics within the Differential Vector Movement category, wherein the differential vector is influenced by neighborhoods.

\begin{tabular}{lllr}
\hline \multicolumn{4}{c}{ Influenced by neighbourhoods } \\
& Acronym & Year & Reference \\
\hline Algorithm Name & BA & 2006 & {$[116]$} \\
\hline Bees Algorithm & BFOA & 2002 & {$[14]$} \\
Biomimicry Of Social Foraging Bacteria for Distributed Optimization & BFOA.1 & 2009 & {$[56]$} \\
Bacterial Foraging Optimization & GELS & 2009 & {$[302]$} \\
Gravitational Emulation Local Search & NCA & 2017 & {$[448]$} \\
Neuronal Communication Algorithm & & & \\
\hline
\end{tabular}

\subsection{Solution Creation}

This category is composed of algorithms that explore the domain search by generating new solutions, not by moving existing ones. This group is a significant ratio (34\%) of all proposals, and includes many classical algorithms like GA [86]. A very widely exploited advantage of these methods is the possibility to adapt the generation method to the particular problem, hence allowing for different possible representations and, therefore, easing its application to a wider range of problems. In the following, we describe the different subcategories that result from the diverse mechanisms by which solutions can be created.

\subsubsection{Creation by Combination}

The most common option to generate a new solution is to combine existing ones. In these algorithms, different solutions are selected and combined using a crossover operator or combining method to give rise to new solutions. The underlying idea is that by combining good solutions, even better solutions can be eventually generated.

The combining method can be specific for the problem to be solved or instead, be conceived for a more general family of problems. In fact, combining methods are usually devised to be adaptable to many different solution representations. As mentioned before, the most popular algorithm in this category is GA [86]. However, many other bio-inspired algorithms exhibit a similar behavior when creating solutions, yet they are inspired by other phenomena, such as Cultural Optimization (CA, [362]) (in the Social Human Behavior category), LA [205] (in the Swarm Intelligence category), Particle Collision Algorithm (PCA, [320], in the chemistry-based category) or Light Ray Optimization (LRO, [313], in the physics-based category). Tables 22, 23, and 24 show the algorithms that rely on combination when creating new solutions along their search. 
Table 22: Nature- and bio-inspired meta-heuristics within the Solution Creation - Combination category (I).

Creation-Combination category (I)

\begin{tabular}{|c|c|c|c|}
\hline Algorithm Name & Acronym & Year & Reference \\
\hline Artificial Beehive Algorithm & $\mathrm{ABA}$ & 2009 & [104] \\
\hline Andean Condor Algorithm & $\mathrm{ACA}$ & 2019 & [107] \\
\hline Artificial Chemical Reaction Optimization Algorithm & ACROA & 2011 & [344] \\
\hline Artificial Ecosystem Algorithm & AEA & 2014 & [74] \\
\hline Artificial Flora Optimization Algorithm & $\mathrm{AFO}$ & 2018 & [401] \\
\hline Artificial Infections Disease Optimization & AIDO & 2016 & [75] \\
\hline Innovative Gunner Algorithm & AIG & 2019 & [421] \\
\hline Anglerfish Algorithm & $\mathrm{AOA}$ & 2019 & [111] \\
\hline Artificial Reaction Algorithm & ARA & 2013 & [345] \\
\hline Asexual Reproduction Optimization & ARO & 2010 & [76] \\
\hline Bacterial-GA Foraging & BGAF & 2007 & [124] \\
\hline Bumblebees & $\mathrm{BB}$ & 2009 & [117] \\
\hline Biogeography Based Optimization & $\mathrm{BBO}$ & 2008 & [77] \\
\hline Bee Colony Optimization & $\mathrm{BCO}$ & 2005 & [120] \\
\hline BeeHive Algorithm & BHA & 2004 & [125] \\
\hline Bees Life Algorithm & BLA & 2018 & [126] \\
\hline Bird Mating Optimization & BMO & 2014 & [78] \\
\hline Barnacles Mating Optimizer & BMO.1 & 2019 & [129] \\
\hline Bean Optimization Algorithm & BOA & 2011 & [79] \\
\hline Bull Optimization Algorithm & BOA.1 & 2015 & [133] \\
\hline Bee System & BS & 1997 & [134] \\
\hline Bar Systems & BS.2 & 2008 & [424] \\
\hline Backtracking Search Optimization & BSO. 3 & 2012 & [425] \\
\hline Bees Swarm Optimization Algorithm & BSOA & 2005 & [139] \\
\hline Bus Transportation Behavior & BTA & 2019 & [357] \\
\hline BrunsVigia Flower Optimization Algorithm & BVOA & 2018 & [403] \\
\hline Black Widow Optimization Algorithm & BWO & 2020 & [141] \\
\hline Cultural Algorithms & $\mathrm{CA}$ & 1999 & [362] \\
\hline Cultural Coyote Optimization Algorithm & CCOA & 2019 & [147] \\
\hline Crystal Energy Optimization Algorithm & CEO & 2016 & [296] \\
\hline Consultant Guide Search & CGS & 2010 & [151] \\
\hline Coral Reefs Optimization & $\mathrm{CRO}$ & 2014 & [12] \\
\hline Chemical Reaction Optimization Algorithm & CRO.1 & 2010 & [346] \\
\hline Cuckoo Search & CS & 2009 & [156] \\
\hline Clonal Selection Algorithm & CSA.1 & 2000 & [428] \\
\hline Dragonfly Swarm Algorithm & DA.1 & 2020 & [160] \\
\hline Dendritic Cells Algorithm & DCA & 2005 & [80] \\
\hline Dolphin Echolocation & DE.1 & 2013 & [161] \\
\hline Duelist Optimization Algorithm & DOA & 2016 & [363] \\
\hline Dialectic Search & DS & 2009 & [431] \\
\hline Election Algorithm & EA & 2015 & [364] \\
\hline Ecogeography-Based Optimization & EBO & 2014 & [82] \\
\hline Eco-Inspired Evolutionary Algorithm & EEA & 2011 & [83] \\
\hline Electromagnetic Field Optimization & $\mathrm{EFO}$ & 2016 & [16] \\
\hline Extremal Optimization & $\mathrm{EO}$ & 2000 & [434] \\
\hline Earthworm Optimization Algorithm & EOA & 2018 & [84] \\
\hline Electimize Optimization Algorithm & EOA.1 & 2011 & [299] \\
\hline Emperor Penguins Colony & EPC & 2019 & [166] \\
\hline
\end{tabular}


Table 23: Nature- and bio-inspired meta-heuristics within the Solution Creation - Combination category (II).

\begin{tabular}{|c|c|c|c|}
\hline \multicolumn{4}{|c|}{ Creation-Combination category (II) } \\
\hline Algorithm Name & Acronym & Year & Reference \\
\hline Evolution Strategies & ES & 2002 & [85] \\
\hline Egyptian Vulture Optimization Algorithm & EV & 2013 & [171] \\
\hline Frog Call Inspired Algorithm & FCA & 2009 & [174] \\
\hline Forest Optimization Algorithm & FOA.1 & 2014 & [400] \\
\hline Genetic Algorithms & GA & 1989 & [86] \\
\hline Golden Ball Algorithm & GBA & 2014 & [367] \\
\hline Galaxy Based Search Algorithm & GBSA & 2011 & [19] \\
\hline Gene Expression & GE & 2001 & [87] \\
\hline Group Leaders Optimization Algorithm & GLOA & 2011 & [369] \\
\hline Honey-Bees Mating Optimization Algorithm & HBMO & 2006 & [187] \\
\hline Hyper-parameter Dialectic Search & HDS & 2020 & [440] \\
\hline Harmony Elements Algorithm & HEA & 2009 & [308] \\
\hline Human Evolutionary Model & HEM & 2007 & [372] \\
\hline Human-Inspired Algorithms & HIA & 2009 & [374] \\
\hline Hysteresis for Optimization & $\mathrm{HO}$ & 2002 & [309] \\
\hline Harmony Search & HS & 2005 & [21] \\
\hline Hypercube Natural Aggregation Algorithm & HYNAA & 2019 & [195] \\
\hline Japanese Tree Frogs Calling Algorithm & JTFCA & 2012 & [199] \\
\hline Immune-Inspired Computational Intelligence & ICI & 2008 & [89] \\
\hline Improved Genetic Immune Algorithm & IGIA & 2017 & [90] \\
\hline Improved Raven Roosting Algorithm & IRRO & 2018 & [196] \\
\hline Invasive Tumor Optimization Algorithm & ITGO & 2015 & [197] \\
\hline Weed Colonization Optimization & IWO & 2006 & [91] \\
\hline Keshtel Algorithm & KA & 2014 & [443] \\
\hline Kaizen Programming & KP & 2014 & [445] \\
\hline Lion Algorithm & LA & 2012 & [202] \\
\hline Laying Chicken Algorithm & LCA & 2017 & [204] \\
\hline Lion Pride Optimizer & LPO & 2012 & [206] \\
\hline Light Ray Optimization & LRO & 2010 & [313] \\
\hline Migrating Birds Optimization & MBO.2 & 2012 & [212] \\
\hline Mosquito Flying Optimization & MFO.1 & 2016 & [217] \\
\hline Marriage In Honey Bees Optimization & MHBO & 2001 & [92] \\
\hline Method of Musical Composition & MMC & 2014 & [316] \\
\hline Mox Optimization Algorithm & MOX & 2011 & [219] \\
\hline Melody Search & MS.1 & 2011 & [317] \\
\hline Natural Aggregation Algorithm & NAA & 2016 & [222] \\
\hline Natural Forest Regeneration Algorithm & NFR & 2016 & [405] \\
\hline Old Bachelor Acceptance & OBA & 1995 & [379] \\
\hline Photosynthetic Algorithm & PA & 1999 & [351] \\
\hline Pity Beetle Algorithm & PBA & 2018 & [229] \\
\hline Polar Bear Optimization Algorithm & PBOA & 2017 & [230] \\
\hline Particle Collision Algorithm & PCA & 2007 & [320] \\
\hline Plant Growth Optimization & PGO & 2008 & [406] \\
\hline Pearl Hunting Algorithm & PHA & 2012 & [449] \\
\hline PopMusic Algorithm & PopMusic & 2002 & [321] \\
\hline Queen-Bee Evolution & QBE & 2003 & [94] \\
\hline Quantum Superposition Algorithm & QSA & 2015 & [322] \\
\hline Red Deer Algorithm & RDA & 2016 & [236] \\
\hline
\end{tabular}


Table 24: Nature- and bio-inspired meta-heuristics within the Solution Creation - Combination category (III).

\section{Creation-Combination category (III)}

\begin{tabular}{llrr}
\hline Algorithm Name & Acronym & Year & Reference \\
\hline Reactive Dialectic Search & RDS & 2017 & {$[451]$} \\
Rain-Fall Optimization Algorithm & RFOA & 2017 & {$[323]$} \\
Rhino Herd Behavior & RHB & 2018 & {$[238]$} \\
Raccoon Optimization Algorithm & ROA & 2018 & {$[240]$} \\
Reincarnation Concept Optimization Algorithm & ROA.1 & 2010 & {$[241]$} \\
Ringed Seal Search & RSS & 2015 & {$[243]$} \\
Shark Search Algorithm & SA & 1998 & {$[244]$} \\
Simulated Annealing & SA.1 & 1989 & {$[23]$} \\
Scientifics Algorithms & SA.2 & 2014 & {$[452]$} \\
SuperBug Algorithm & SuA & 2012 & {$[95]$} \\
Simulated Bee Colony & SBC & 2009 & {$[245]$} \\
Snap-Drift Cuckoo Search & SDCS & 2016 & {$[248]$} \\
Self-Defense Mechanism Of The Plants Algorithm & SDMA & 2018 & {$[413]$} \\
Social Engineering Optimization & SEO & 2017 & {$[453]$} \\
Sheep Flock Heredity Model & SFHM & 2001 & {$[97]$} \\
Shuffled Frog-Leaping Algorithm & SFLA & 2006 & {$[249]$} \\
Saplings Growing Up Algorithm & SGA.1 & 2007 & {$[412]$} \\
Search Group Algorithm & SGA.2 & 2015 & {$[455]$} \\
Swine Influenza Models Based Optimization & SIMBO & 2013 & {$[98]$} \\
Sonar Inspired Optimization & SIO & 2017 & {$[329]$} \\
Self-Organizing Migrating Algorithm & SOMA & 2004 & {$[99]$} \\
Simple Optimization & SOPT & 2012 & {$[456]$} \\
Strawberry Plant Algorithm & SPA & 2014 & {$[414]$} \\
Salp Swarm Algorithm & SSA.2 & 2017 & {$[264]$} \\
Tree Growth Algorithm & TGA.1 & 2019 & {$[415]$} \\
The Great Deluge Algorithm & TGD & 1993 & {$[458]$} \\
Small World Optimization & SWO & 2006 & {$[457]$} \\
Virulence Optimization Algorithm & VOA & 2016 & {$[101]$} \\
Virus Optimization Algorithm & VOA.1 & 2009 & {$[279]$} \\
Viral Systems Optimization & VSO & 2008 & {$[280]$} \\
Wasp Colonies Algorithm & WCA & 1991 & {$[10]$} \\
Water Flow-Like Algorithms & WFA & 2007 & {$[339]$} \\
Water Flow Algorithm & WFA.1 & 2007 & {$[340]$} \\
Wasp Swarm Optimization & WSO & 2005 & {$[286]$} \\
Ying-Yang Pair Optimization & YYOP & 2016 & {$[418]$} \\
\hline & & & \\
& & & \\
\hline
\end{tabular}




\subsubsection{Creation by Stigmergy}

Another popular option of creating new solutions relies on stigmergy, namely, an indirect communication and coordination between the different solutions or agents used to create new solutions. This communication is usually done using an intermediate structure, with information obtained from the different solutions, used to generate new solutions oriented towards more promising areas of the search space. This is indeed the search mechanism used in the most representative algorithm of this category, ACO [7], which is inspired by the foraging mechanism of ant colonies. Each ant of the colony describes a trajectory over a graph representation of the search space of the problem at hand, and leaves a trace of pheromone along its way whose intensity depends, in part, on the fitness value corresponding to the solution encoded by the trajectory of the ant. In subsequent iterations, new solutions are generated, dimension by dimension, considering the pheromones trail left by preceding ants, enforcing the search around the most promising values for each dimension.

Table 25 lists the reviewed algorithms that employ stigmergy when creating new solutions. This is a reduced list when comparing with preceding categories, with the majority of the algorithms relying on Swarm Intelligence among insects (similarly to ACO). However, other algorithms inspired in physics have also a stigmertic behavior when producing new solutions, such as methods inspired by water flow dynamics [341] and the natural formation of rivers [325].

Table 25: Nature- and bio-inspired meta-heuristics within the Solution Creation - Stimergy category.

\begin{tabular}{lllr}
\hline \multicolumn{4}{c}{ Solution Creation - Stimergy } \\
\hline Algorithm Name & Acronym & Year & Reference \\
\hline Ant Colony Optimization & ACO & 1996 & {$[6]$} \\
Bee System & BS.1 & 2002 & {$[135]$} \\
Hammerhead Shark Optimization Algorithm & HSOA & 2019 & {$[194]$} \\
Intelligence Water Drops Algorithm & IWD & 2009 & {$[312]$} \\
River Formation Dynamics & RFD & 2007 & {$[325]$} \\
Termite Hill Algorithm & TA & 2012 & {$[272]$} \\
Virtual Ants Algorithm & VAA & 2006 & {$[276]$} \\
Virtual Bees Algorithm & VBA & 2005 & {$[277]$} \\
Water-Flow Algorithm Optimization & WFO & 2011 & {$[341]$} \\
\hline
\end{tabular}

\section{Taxonomies Analysis: Comparison and More Influential Algorithms}

We now proceed by critically examining the reviewed literature as per the different taxonomies proposed in this overview. First, we are going to study the similarities between the results of the classifications following each taxonomy. Later, we identify the most influential algorithms over the rest, based on the behavior of the algorithms. 


\subsection{Comparison Between the Two Taxonomies}

Comparing the different taxonomies to each other and the algorithms falling in each of their categories, it can be observed that there is not a strong relationship between them. Interestingly, this unveils that features characterizing one algorithm are loosely associated with its inspirational model. For instance, algorithms inspired by very different concepts such as the gravitational forces (GFA, [303]) or animal evolution (ABO, [106]) exhibit a significant similarity with PSO [2]. This statement is supported by the fact that, in the second taxonomy, each category is composed by algorithms that, as per the first taxonomy, are inspired by diverse phenomena. The contrary also holds in general: proposals with very similar natural inspiration fall in the same category of the first taxonomy (as expected), but their search procedures differ significantly from each other, thereby being classified in different categories of the second taxonomy. An illustrative example is the Delphi Echolocation algorithm (DE, [161]) and the Dolphin Partner Optimization [163]. Both are inspired in the same animal (dolphin) and its mechanism to detect fishes (echolocation), but they are very different algorithms: the former creates new solutions by combination, whereas the latter resembles closely the movement performed in the PSO solver, mainly guided by the best solution.

In this same line of reasoning, the largest subcategory of the second taxonomy (Differential Vector Movements guided by representative solutions) not only contains more than half of the reviewed algorithms (55\%), but it also comprises algorithms from all the different categories in the first taxonomy: Social Human Behavior (as Anarchic Society Optimization, ASO, [27]), microorganisms (Bacterial Colony Optimization, [121]), Physics/Chemistry category (correspondingly, Fireworks Algorithm Optimization, FAO, [435]), Breeding-based Evolution (as Variable Mesh Optimization, VMO [100]), or even Plants-Based (such as Flower Pollination Algorithm, FPA [404]). This inspirational diversity is not exclusive to this subcategory. Others, such as Solution Creation, also include algorithms relying on an heterogeneity of natural concepts.

Considering the previous examples, it is clear that the real behavior of the algorithm is much more informative than its natural or biological inspiration. Even more, we have observed that in our first proposed taxonomy, built upon the review of more than four hundred proposals, the huge diversity of inspirational sources does not correspond with the lower number of algorithmic behaviors on which our second taxonomy is based. This observation is in accordance with previous works in the literature, which have put to question whether the novelty in the natural inspiration of the algorithm actually yields different algorithms that could produce competitive results [460, 461].

We further elaborate on the above statement: our literature analysis revealed that the majority of proposals (more than a half, 52\%) generates new solutions based on differential vector forces over existing ones, as in the classical PSO or DE. A complementary analysis can be done by departing from this observation towards discriminating which of the classical algorithms (PSO, DE, GA, ACO, ABC or SA) can be declared to be most similar to modern approaches. The results of this analysis are conclusive: $30 \%$ of all reviewed algorithms (122 out of 412) were found to be so influenced by classical algorithms that, without their biological inspiration, they could be regarded as incremental variants. The other 290 solvers 
(about 70\%) have enough differences to be considered a new proposal by themselves, instead of another version of an existing algorithm.

Table 26: Percentages of similar algorithms in the reviewed literature.

\begin{tabular}{lrr}
\hline Classical algorithm & Number of papers with similar algorithms & Percentage over the total \\
\hline PSO & 57 & $13.83 \%$ \\
DE & 24 & $5.82 \%$ \\
GA & 24 & $5.82 \%$ \\
ACO & 7 & $1.70 \%$ \\
ABC & 7 & $1.70 \%$ \\
SA & 3 & $0.73 \%$ \\
\hline Total & 122 & $29.60 \%$ \\
\hline
\end{tabular}

\subsection{Identification of the Most Influential Algorithms}

In order to know of which are the most influential reference algorithms used to design other bio-inspired algorithms, we have grouped together reviewed proposals that could be considered to be versions of the same classical algorithm. Figure 5.2 shows the classification of each algorithm based on its behavior, and the number of proposals in each classification are summarized in Table 26.

Very insightful conclusions can be drawn from this grouping. To begin with, in Table 26 the most influential algorithm was identified to be PSO, appearing in $14 \%$ of the reviewed literature (which corresponds to $46 \%$ of the proposals that were clearly based on a previous algorithm). This bio-inspired solver is one of the most prominent and historically acknowledged algorithms in the Swarm Intelligence category, and is the reference of many bio-inspired algorithms contributed since its inception. The simplicity of this algorithm and its ability to reach an optimum quickly - as has been comparatively assessed in many application scenarios, see e.g. [66, 67] - have inspired many authors to create new metaheuristics characterized by similar solution movement dynamics to those defined by PSO. Thus, many algorithms whose authors claim to simulate the behavior of a biological system eventually perform their search process through movements strongly influenced by PSO (in some cases, without any significant difference).

The second and third most influential algorithms are GA, a very classic algorithm, and DE, a well-know algorithm whose natural inspiration resides only as the evolution of a population. Both have been used by around $6 \%$ of all reviewed nature-inspired algorithms, and they are the most representative approach in the Evolutionary Algorithms category. The search mechanism of GA is solution creation by combination, and the search mechanism of DE is to create new solutions with a lineal combination of existing ones in the population, which is used by $6 \%$ of all reviewed proposals, maybe by its superior performance reported for many optimization problems [38]. 


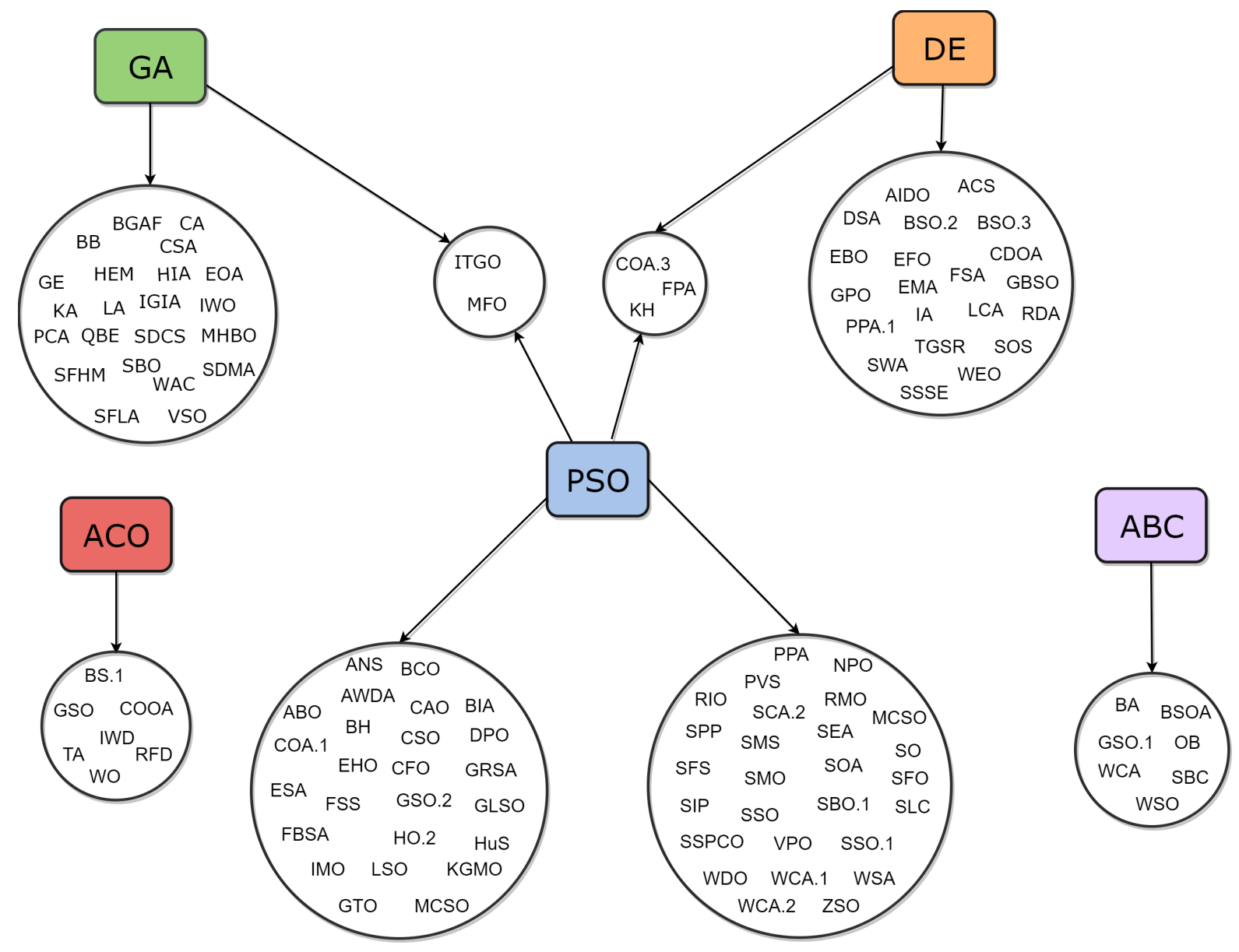

Figure 6: Classification of proposals by its original algorithm. 
When inspecting the influential approaches from a higher perspective, two are the categories whose algorithms have been more frequently used to create new nature-based algorithms. The first one is Swarm Intelligence: about $17 \%$ of all studied nature inspired algorithms are variations of SI algorithms (PSO, ACO, and ABC). The second one is shared between Evolutionary Algorithms and GA, whose represented algorithms are both used in $6 \%$ of the reviewed cases. It is noteworthy to highlight that it appears that the influence of more classic algorithms like GA and SA is declining when compared to other algorithms, such as DE and PSO.

In summary, although in the last years many nature-inspired algorithms have been proposed by the community and their number grows steadily every year, more than half of the proposals reviewed in our work are incremental, minor versions of only three very classical algorithms (PSO, DE, and GA). We therefore conclude that a huge number of natural and biological sources of inspiration used so far to justify the design of new optimization solvers have not led to significantly disruptive algorithmic behaviors. This closing note will be at the heart of our critical analysis exposed in the next section.

\section{Lessons Learnt and Critical Analysis: Recommendations on Research Practices}

After reviewing the proposals, we have extracted several issues that we consider to be challenges and recommendations that the community working on nature- and bio-inspired optimization should deal with in forthcoming years. We next outline them in no particular order:

- The behavior is more relevant than the natural inspiration: As was exposed in Section 5, the current literature is flooded with a huge number of nature- and bio-inspired algorithms. However, as has been spotted by our proposed taxonomies, several algorithms belonging to categories with different sources of inspiration result to be very similar in terms of behavior. This disparity is a controversial topic in recent years [32,460]. Therefore, we call for more research efforts around the design of optimization algorithms that focus on their behavior and properties (e.g., good performance, simplicity, ability to run it in parallel or their suitability to a specific type of problems) rather than on new inspiration sources.

- Nature-based terminology can make it more difficult to understand the proposal: A great deal of papers presenting new bio-inspired solvers are difficult to understand and replicate due to the extended usage of vocabulary related to the natural source of inspiration. It is logical to use the semantic of the biological or natural domain, but to an extent. It would be desirable that the description of the algorithm could be defined in an inspiration-agnostic fashion, resorting to mathematical terms to describe each component, agent and/or phase of the optimization process (e.g. optimum/a, individuals or solutions). An excessive usage of the domain terminology (without explicitly indicating the correspondences) could make it difficult to follow the details of the algorithm for researchers not acquainted with such a terminology. To overcome this issue, the correspondence between the domain terminology and the optimization terminology should be explicitly indicated. 
- Good comparisons are crucial for new proposals: The lack of fair comparisons is another important drawback of many proposals published to date. When new algorithms are proposed, unfortunately, many of them are only compared to very basic and classical algorithms (such as GA or PSO). These algorithms have been widely surpassed by more advanced versions over the years which, so obtaining better performance than naive version of classical algorithms is relatively easy to achieve, and it does not imply a competitive performance [461]. In some cases, the proposed algorithm is compared to similar algorithms but not with competitive algorithms outside that semantic niche [461, 462]. This methodological practice must be regarded as a very serious barrier for their application to real-world problems. We encourage researchers to increase the algorithms used in their experimental section, including more competitive or state-of-the-art algorithms: until they are proven to be competitive in respect to the state of the art, new nature- and bio-inspired solvers will not be used in practice either will attract enough attention of the research community.

- Many proposals have a very limited influence: By examining in depth the historical trajectory followed by each reviewed algorithm, an intriguing trend is revealed: a fraction of the proposals has a very limited influence in new papers after the original publication. For them, there is almost no new papers with improved versions, or applying it to new problems. Fortunately, other few algorithms have a stronger influence. In view of this dichotomy, the researchers should evaluate their proposals to diverse problems, including widely acknowledged benchmark functions and real-world practical problems, to grasp the interest of the community in considering their proposed algorithms for tackling other applications.

- The interest of making source code available: Related to the previous one, it is very interesting, in order to gain more visibility, to make the source code of the proposed algorithm available for the community. It is true that the paper presenting the new algorithm should be detailed enough to allow for a clean implementation of the proposal from the provided specification. However, it is widely acknowledged that, in many occasions, there are important details that even though they have a strong influence on the results, are not remarked in the description [463, 464]. A publicly available reference implementation could not only improve its visibility, but could also offer other researchers the chance to undertake more thorough performance comparisons. In addition, there are a huge number of software frameworks for Evolutionary Computation and Swarm Intelligence programmed in different languages (such as C++, Java, Matlab, or Python), some of them very popular in the current research landscape. To cite a few: Evolutionary Computation Framework (ECF) ${ }^{1}$ and ParadisEO [465] in C++; jMetal [466] and MOEA ${ }^{2}$ in Java; NiaPy[467], jMetalPy [468] and PyGMO ${ }^{3}$ in Python; or PlatEMO [469] in Matlab, among others. Each of them implements the most popular algorithms (GA, DE, PSO, ABC, ...). A reference implementation could also favor the inclusion of the proposal in frameworks as the ones exemplified previously. Otherwise, different implementations of the allegedly same algorithm could produce diverging results from the

\footnotetext{
${ }^{1}$ http://ecf.zemris.fer.hr/

${ }^{2}$ http://moeaframework.org/

${ }^{3}$ http://esa.github.io/pygmo/index.html
} 
original proposal (in part due to the ambiguity of the description).

- The role of bio-inspired algorithms in competitions: Finally, we also stress on the fact that metaheuristic algorithms that have scored best in many competitions are far from being biologically inspired, although some of them retain their nature-inspired roots (mostly, DE) [38]. This fact was expected for the lack of good methodological practices when comparing nature- and bio-inspired algorithms, which was pointed out previously in our analysis. This issue has not encouraged participants in competitions to embrace them as reference algorithms to design better solvers. The rising trend of the community to generate an ever-growing number of bio-inspired proposals can be counterproductive and deviate efforts towards developments of a reduced number of proposals but with a better performance.

- Work in more specific challenges: Most proposals aim to address general single-objective optimization problems. However, other types of optimization problems still deserve further attention from the community, unfolding vast opportunities for the development of special flavors of nature- and bio-inspired algorithms as the ones collected in our taxonomies:

- Dynamic and stochastic optimization, problems in which the problem definition varies over time.

- Multi- and many-objective optimization, wherein the goal is to simultaneously optimize several conflicting objectives.

- Multimodal optimization, in which there may be several global optima to be found and retained during the search.

- Large-Scale Global Optimization, in which the number of variables (dimensionality of the search space) is huge, in the order of thousands.

- Memetic Algorithms, where the algorithmic proposal is combined with other search techniques to further improve its performance.

- Parameter tuning, which refers to the search for the values of the parameters of the optimization algorithm itself that lead to its best search performance.

- Parameter adaptation, i.e., the design of adaptive parameters that allow solver to adapt themselves to the problem during the search.

We suggest [32] to the reader interested in further information and prospects on the future of the above type of problems.

- Hybridization might produce new algorithmic behaviors: In our bibliographic study, we have noted the tendency in the literature to propose combinations (hybridizations) of two or more nature- and bio-inspired algorithms into a single solver [470, 471]. However, in the different proposals, a solid proof is required to verify that the results compensate for the increase in complexity when compared to existing approaches. When this proof is eventually achieved, the community should gradually incorporate these new hybrid approaches into existing taxonomies. This could require widening the criteria 
followed in taxonomies to also account for the mixture of algorithmic behaviors present in hybrid search techniques. Taxonomies reported to date do not explicitly take into account this possibility. Once hybrid schemes are verified to provide significant gains over traditional methods, it will be time for the research community to start modifying the existing taxonomies to reflect their importance in the field.

\section{Conclusions}

Nature and biological organisms have been a source of inspiration of many optimization algorithms. During the last years, this family of solvers has grown considerably in size, achieving unseen levels of diversity with regard to their source of inspiration. This explosion of literature has made it difficult for the community to appraise the general trajectory followed by the field, which is a necessary step towards identifying research trends and challenges of scientific value and practical impact. Some efforts have been dedicated so far towards classifying the state of the art on nature- and bio-inspired optimization in a taxonomy with well-defined criteria, allowing researchers to classify existing algorithms and newly proposed schemes. Unfortunately, the few attempts at furnishing this desired taxonomy have not succeeded through the years, relying mostly on the source of inspiration (metaphor) or in taxonomies with narrow algorithmic coverage.

In this work, we have reviewed more than four hundred nature- and bio-inspired algorithms, and classified them in two taxonomies that group the different proposals in categories and subcategories. The first proposed taxonomy considers its source of inspiration, whereas the second taxonomy discriminates among algorithm by their algorithmic behavior, namely, by the procedure by which new candidate solutions to the optimization problem are generated. Remarkably, our second taxonomy leaves aside any aspect related to the source of inspiration behind the design of the algorithm to strictly focus on algorithmic aspects of its search procedure. We have provided clear descriptions of the classification criterion, illustrative examples and an enumeration of the reviewed nature- and bio-inspired approaches that fall within each of the categories of both proposed taxonomies.

Once the taxonomies have been presented and populated, our study has also critically examined the reviewed literature by identifying and discussing on the similarities and differences among them. We have concluded that a very loose connection exists between the natural inspiration of an algorithm and its algorithmic behavior. Likewise, many algorithms, even if claiming to be inspired by very different natural and biological phenomena, result to be algorithmically more similar than one could expect as per their design rationale. Even more serious is the noted fact that more than $55 \%$ of all proposals follow a very similar behavior, namely, the exploration of the search space by moving a reference solution with a differential vector towards the current best solution. Yet another finding that goes in this line of discussion: $25 \%$ of the reviewed proposals were identified to be versions of classical algorithms such as PSO, DE, or GA. Specially PSO, with more than $13 \%$ of the nature- 
and bio-inspired algorithms proposed in the last years that can be regarded as versions of this solver. To a lesser albeit also illustrative extent, $6 \%$ of the studied proposals were versions of GA. These findings bring light to the longstanding discussion held within the nature- and bio-inspired community around the questionable algorithmic contributions of recent advances in the field.

On a note summarizing our above challenges and recommendations, we stress on our main three conclusions:

1. The growing number of nature- and bio-inspired proposals must be regarded as a symptomatic fact of the vivid status of this field [32], as well as a clear exponent of the possibilities brought by Nature to solve complex optimization problems.

2. Its evolution suggest that research efforts should aim at devising new algorithms with truly new behavioral differences with respect to the state of the art, providing verifiable evidences of a superior performance in practical problems.

3. Good methodological practices must be followed in forthcoming studies when designing, describing, and comparing new algorithms.

All in all, there are exciting times for research on nature- and bio-inspired optimization, which should depart from a consensus on which research avenues should be pursued collectively by the community. We hope that the double taxonomy proposed in this overview and the critical analysis made thereafter take a sensible step in this direction, and contribute to achieving the scientific soundness and technical rigor that this field deserves.

\section{Acknowledgments}

This work was supported by grants from the Spanish Ministry of Science and the European Fund (FEDER) under projects TIN2016-81113R, and TIN2017-89517-P. Javier Del Ser would like to thank the Basque Government for its support through the ELKARTEK and EMAITEK funding programs.

\section{Compliance with Ethical Standards}

Ethical approval: This article does not contain any studies with human participants or animals performed by any of the authors.

\section{Conflict of Interests}

AH is Editor-in-Chief, and FH is editorial board member of Cognitive Computation. They had no involvement in any aspect of the decision making on this paper. 


\section{References}

[1] Yang XS, Xiao R, Karamanoglu M, Cui Z, Gandomi AH, editors. Swarm Intelligence and Bio-Inspired Computation: Theory and Applications. Elseview; 2013.

[2] Eberhart R, Kennedy J. A new optimizer using particle swarm theory. In: MHS'95. Proceedings of the Sixth International Symposium on Micro Machine and Human Science; 1995. p. 39-43.

[3] Yang XS. In: A New Metaheuristic Bat-Inspired Algorithm. Springer; 2010. p. 65-74.

[4] Yang XS. Firefly Algorithms for Multimodal Optimization. In: Stochastic Algorithms: Foundations and Applications; 2009. p. 169-178.

[5] Saremi S, Mirjalili S, Lewis A. Grasshopper Optimisation Algorithm: Theory and application. Advances in Engineering Software. 2017;105:30-47.

[6] Dorigo M, Maniezzo V, Colorni A. The Ant System: Optimization by a colony of cooperating agents. IEEE Transactions on Systems, Man, and Cybernetics, Part B (Cybernetics). 1996;26(1):29-41.

[7] Dorigo M, Stützle T. Ant Colony Optimization. MIT Press; 2004.

[8] Karaboga D, Basturk B. A powerful and efficient algorithm for numerical function optimization: artificial bee colony (ABC) algorithm. Journal of Global Optimization. 2007;39(3):459-471.

[9] Mirjalili S. Dragonfly algorithm: a new meta-heuristic optimization technique for solving single-objective, discrete, and multi-objective problems. Neural Computing and Applications. 2016;27(4):1053-1073.

[10] Theraulaz G, Goss S, Gervet J, Deneubourg JL. Task differentiation in Polistes wasp colonies: a model for self-organizing groups of robots. Proceedings of the First International Conference on Simulation of Adaptive Behavior : From Animals to Animates. 1991;p. 346-355.

[11] Mirjalili S, Lewis A. The Whale Optimization Algorithm. Advances in Engineering Software. 2016;95:51-67.

[12] Salcedo-Sanz S, Del Ser J, Landa-Torres I, Gil-López S, Portilla-Figueras J. The coral reefs optimization algorithm: a novel metaheuristic for efficiently solving optimization problems. The Scientific World Journal. 2014;2014, Article ID 739768,15 pages.

[13] Hossein Gandomi A, Hossein Alavi A. Krill herd: A new bio-inspired optimization algorithm. Communications in Nonlinear Science and Numerical Simulation. 2012;17(12):4831-4845. 
[14] Lui Y, Passino KM. Biomimicry of Social Foraging Bacteria for Distributed Optimization: Models, Principles, and Emergent Behaviors. Journal of Optimization theory and applications. 2002;115(3):603-628.

[15] Jiang Q, Wang L, Hei X, Fei R, Yang D, Zou F, et al. Optimal approximation of stable linear systems with a novel and efficient optimization algorithm. In: Proceedings of the IEEE Congress on Evolutionary Computation, CEC; 2014. p. $840-844$.

[16] Abedinpourshotorban H, Shamsuddin SM, Beheshti Z, Jawawi DNA. Electromagnetic field optimization: A physics-inspired metaheuristic optimization algorithm. Swarm and Evolutionary Computation. 2016;26:8-22.

[17] Ilker Birbil S, Fang SC. An Electromagnetism-like Mechanism for Global Optimization. Journal of Global Optimization. 2003;25(3):263-282.

[18] Rashedi E, Nezamabadi-Pour H, Saryazdi S. GSA: A Gravitational Search Algorithm. Information Sciences. 2009;179(13):2232-2248.

[19] Shah-Hosseini H. Principal components analysis by the galaxy-based search algorithm: a novel metaheuristic forcontinuous optimisation. International Journal of Computational Science and Engineering. 2011;6(1):132-140.

[20] Zhou Y, Luo Q, Liu J. Glowworm Swarm Optimization For Optimization Dispatching System Of Public Transit Vehicles. Journal of Theoretical and Applied Information Technology. 2013;52:205-210.

[21] Lee KS, Geem ZW. A new meta-heuristic algorithm for continuous engineering optimization: harmony search theory and practice. Computer methods in applied mechanics and engineering. 2005;194:3902-3933.

[22] Abdechiri M, Meybodi MR, Bahrami H. Gases Brownian Motion Optimization: an Algorithm for Optimization (GBMO). Applied Soft Computing. 2013;13:2932-2946.

[23] Kirkpatrick S, Gelatt CD, P VM. Optimization by Simulated Annealing. Science. 1989;220(4598):671-680.

[24] Moosavian N, Roodsari BK. Soccer league competition algorithm: A novel meta-heuristic algorithm for optimal design of water distribution networks. Swarm and Evolutionary Computation. 2014;17:14-24.

[25] Kashan AH. League Championship Algorithm (LCA): An algorithm for global optimization inspired by sport championships. Applied Soft Computing. 2014;16:171-200.

[26] Shi Y. Brain Storm Optimization Algorithm. In: Advances in Swarm Intelligence; 2011. p. 303-309.

[27] Ahmadi-Javid A. Anarchic Society Optimization: A human-inspired method. In: 2011 IEEE Congress of Evolutionary Computation (CEC); 2011. p. 2586-2592. 
[28] Atashpaz-Gargari E, Lucas C. Imperialist competitive algorithm: An algorithm for optimization inspired by imperialistic competition. In: 2007 IEEE Congress on Evolutionary Computation; 2007. p. 4661-4667.

[29] Pintea CM. In: Bio-inspired Computing. Springer Berlin Heidelberg; 2014. p. 3-19.

[30] Mahdavi S, Shiri ME, Rahnamayan S. Metaheuristics in large-scale global continues optimization: A survey. Information Sciences. 2015;295:407-428.

[31] Sörensen K. Metaheuristics-the metaphor exposed. International Transactions In Operational Research. $2015 ; 22(1): 3-18$.

[32] Del Ser J, Osaba E, Molina D, Yang XS, Salcedo-Sanz S, Camacho D, et al. Bio-inspired computation: Where we stand and what's next. Swarm and Evolutionary Computation. 2019;48:220 - 250.

[33] Fister Jr I, Mlakar U, Brest J, Fister I. A new population-based nature-inspired algorithm every month: is the current era coming to the end. In: Proceedings of the 3rd Student Computer Science Research Conference. University of Primorska Press; 2016. p. 33-37.

[34] Weyland D. A critical analysis of the harmony search algorithm - how not to solve sudoku. Operations Research Perspectives. 2015;2:97-105.

[35] Saka MP, Hasançebi O, Geem ZW. Metaheuristics in structural optimization and discussions on harmony search algorithm. Swarm and Evolutionary Computation. 2016;28:88-97.

[36] Kar AK. Bio inspired computing - A review of algorithms and scope of applications. Expert Systems with Applications. 2016;59:20-32.

[37] Xiong N, Molina D, Ortiz ML, Herrera F. A walk into metaheuristics for engineering optimization: principles, methods and recent trends. International Journal of Computational Intelligence Systems, 2015. 2015;8(4):606-636.

[38] Molina D, LaTorre A, Herrera F. An Insight into Bio-inspired and Evolutionary Algorithms for Global Optimization: Review, Analysis, and Lessons Learnt over a Decade of Competitions. Cognitive Computation. 2018;10(4):517-544.

[39] Zavala GR, Nebro AJ, Luna F, Coello Coello CA. A survey of multi-objective metaheuristics applied to structural optimization. Structural and Multidisciplinary Optimization. 2014;49(4):537-558.

[40] Yang XS, Chien SF, Ting TO. Chapter 1 - Bio-Inspired Computation and Optimization: An Overview. In: Yang XS, Chien SF, Ting TO, editors. Bio-Inspired Computation in Telecommunications. Morgan Kaufmann; 2015. p. 1 - 21. 
[41] Beni G, Wang J. Swarm Intelligence in Cellular Robotic Systems. In: Dario P, Sandini G, Aebischer P, editors. Robots and Biological Systems: Towards a New Bionics?; 1993. p. 703-712.

[42] Fong S. 18 - Opportunities and Challenges of Integrating Bio-Inspired Optimization and Data Mining Algorithms. In: Yang XS, Cui Z, Xiao R, Gandomi AH, Karamanoglu M, editors. Swarm Intelligence and Bio-Inspired Computation. Elsevier; 2013. p. $385-402$.

[43] Del Ser J, Osaba E, Sanchez-Medina JJ, Fister I. Bioinspired computational intelligence and transportation systems: a long road ahead. IEEE Transactions on Intelligent Transportation Systems, in press. 2019;.

[44] del Valle Y, Venayagamoorthy GK, Mohagheghi S, Hernandez J, Harley RG. Particle Swarm Optimization: Basic Concepts, Variants and Applications in Power Systems. IEEE Transactions on Evolutionary Computation. 2008;12(2):171-195.

[45] Dressler F, Akan OB. A survey on bio-inspired networking. Computer Networks. 2010;54(6):881 - 900.

[46] José-García A, Gómez-Flores W. Automatic clustering using nature-inspired metaheuristics: A survey. Applied Soft Computing. 2016;41:192-213.

[47] Alsalibi B, Venkat I, Subramanian KG, Lutfi SL, Wilde PD. The Impact of Bio-Inspired Approaches Toward the Advancement of Face Recognition. ACM Computing Surveys. 2015;48(5):1-33.

[48] García-Godoy MJ, López-Camacho E, García-Nieto J, Del Ser J, Nebro AJ, Aldana-Montes JF. Bio-inspired optimization for the molecular docking problem: State of the art, recent results and perspectives. Applied Soft Computing. 2019;79:30-45.

[49] Kolias C, Kambourakis G, Maragoudakis M. Swarm intelligence in intrusion detection: A survey. Computers and Security. 2011;30(8):625-642.

[50] Banks A, Vincent J, Anyakoha C. A review of particle swarm optimization. Part I: background and development. Natural Computing. 2007;6(4):467-484.

[51] Neri F, Tirronen V. Recent advances in differential evolution: a survey and experimental analysis. Artificial Intelligence Review. 2010;33(1):61-106.

[52] Das S, Suganthan PN. Differential Evolution: A Survey of the State-of-the-Art. IEEE Transactions on Evolutionary Computation. 2011;15(1):4-31. 
[53] Das S, Mullick SS, Suganthan PN. Recent advances in differential evolution - An updated survey. Swarm and Evolutionary Computation. 2016;27:1 - 30 .

[54] Karaboga D, Gorkemli B, Ozturk C, Karaboga N. A comprehensive survey: artificial bee colony (ABC) algorithm and applications. Artificial Intelligence Review. 2014;42(1):21-57.

[55] Bitam S, Batouche M, Talbi E. A survey on bee colony algorithms. In: 2010 IEEE International Symposium on Parallel Distributed Processing, Workshops and Phd Forum (IPDPSW); 2010. p. 1-8.

[56] Das S, Biswas A, Dasgupta S, Abraham A. In: Bacterial Foraging Optimization Algorithm: Theoretical Foundations, Analysis, and Applications. Berlin, Heidelberg: Springer Berlin Heidelberg; 2009. p. 23-55.

[57] Yang XS, He X. Bat Algorithm: Literature Review and Applications. International Journal of Bio-Inspired Computation. 2013;5(3):141-149.

[58] Bonabeau E, Dorigo M, Théraulaz G. Swarm intelligence: from natural to artificial systems. Oxford University press; 1999.

[59] Yang XS. Nature-Inspired Optimization Algorithms. Elsevier; 2014.

[60] Das S, Abraham A, Konar A. In: Particle Swarm Optimization and Differential Evolution Algorithms: Technical Analysis, Applications and Hybridization Perspectives. Springer Berlin Heidelberg; 2008. p. 1-38.

[61] Elbeltagi E, Hegazy T, Grierson D. Comparison among five evolutionary-based optimization algorithms. Advanced Engineering Informatics. 2005;19(1):43-53.

[62] Pazhaniraja N, Paul PV, Roja G, Shanmugapriya K, Sonali B. A study on recent bio-inspired optimization algorithms. In: 2017 Fourth International Conference on Signal Processing, Communication and Networking (ICSCN); 2017. p. $1-6$.

[63] Krömer P, Platoš J, Snášel V. Nature-Inspired Meta-Heuristics on Modern GPUs: State of the Art and Brief Survey of Selected Algorithms. International Journal of Parallel Programming. 2014;42(5):681-709.

[64] Piotrowski AP, Napiorkowski M, Napiorkowski JJ, Rowinski PM. Swarm Intelligence and Evolutionary Algorithms: performance versus speed. Information Sciencies. 2017;384:34-85.

[65] El-Abd M. Performance assessment of foraging algorithms vs. evolutionary algorithms. Information Sciences. 2012;182(1):243-263. 
[66] Chouikhi N, Ammar B, Hussain A, Alimi AM. Bi-level multi-objective evolution of a Multi-Layered Echo-State Network Autoencoder for data representations. Neurocomputing. 2019;341:195-211.

[67] Chouikhi N, Ammar B, Rokbani N, Alimi AM. PSO-based analysis of Echo State Network parameters for time series forecasting. Applied Soft Computing. 2017;55:211-225.

[68] Fister jr I, Yang XS, Fister I, Brest J, Fister D. A Brief Review of Nature-Inspired Algorithms for Optimization. Elektrotehniski Vestnik. 2013;80(3):1-7.

[69] Baskaran A, Balaji N, Basha S, Vengattaraman T. A survey of nature inspired algorithms. International Journal of Applied Engineering Research. 2015;10:19313-19324.

[70] Rajakumar R, Dhavachelvan P, Vengattaraman T. A survey on nature inspired meta-heuristic algorithms with its domain specifications. In: 2016 International Conference on Communication and Electronics Systems (ICCES); 2016. p. 1-6.

[71] Kumar Kar A. Bio inspired computing - A review of algorithms and scope of applications. Expert Systems With Applications. 2016;59:20-32.

[72] Chu X, Wu T, Weir JD, Shi Y, Niu B, Li L. Learning-interaction-diversification framework for swarm intelligence optimizers: a unified perspective. Neural Computing and Applications. 2018;p. 1-21.

[73] Braik MS. Chameleon Swarm Algorithm: A bio-inspired optimizer for solving engineering design problems. Expert Systems with Applications. 2021;174:114685.

[74] Adham M, Bentley P. An Artificial Ecosystem Algorithm applied to static and Dynamic Travelling Salesman Problems; 2015. p. 149-156.

[75] Huang G. Artificial infectious disease optimization: A SEIQR epidemic dynamic model-based function optimization algorithm. Swarm and Evolutionary Computation. 2016;27:31-67.

[76] Farasat A, Menhaj MB, Mansouri T, Sadeghi Moghadamd MR. ARO: A new model-free optimization algorithm inspired from asexual reproduction. Applied Soft Computing. 2010;10(4):1284-1292.

[77] Simon D. Biogeography-Based Optimization. IEEE Transactions on Evolutionary Computation. 2008;12(6):702-713.

[78] Askarzadeh A. Bird mating optimizer: An optimization algorithm inspired by bird mating strategies. Communications in Nonlinear Science and Numerical Simulation. 2014;19(4):1213 - 1228.

[79] Zhang X, Sun B, Mei T, Wang R. Post-disaster restoration based on fuzzy preference relation and Bean Optimization Algorithm. In: 2010 IEEE Youth Conference on Information, Computing and Telecommunications; 2010. p. 271-274. 
[80] Greensmith J, Aickelin U, Cayzer S. Introducing dendritic cells as a novel immune-inspired algorithm for anomaly detection. In: International Conference on Artificial Immune Systems. Springer; 2005. p. 153-167.

[81] Price K, Storn R. A simple and efficient heuristic for global optimization over continuous spaces. Journal of Global Optimization. 1997;11(4):341-359.

[82] Zheng YJ, Ling HF, Xue JY. Ecogeography-based optimization: Enhancing biogeography-based optimization with ecogeographic barriers and differentiations. Computers and Operations Research. 2014;50:115-127.

[83] Parpinelli RS, Lopes HS. An eco-inspired evolutionary algorithm applied to numerical optimization. In: 2011 Third World Congress on Nature and Biologically Inspired Computing; 2011. p. 466-471.

[84] Wang GG, Deb S, dos Santos Coelho L. Earthworm optimisation algorithm: a bio-inspired metaheuristic algorithm for global optimisation problems. IJBIC. 2018;12(1):1-22.

[85] Beyer HG, Schwefel HP. Evolution strategies - A comprehensive introduction. Natural Computing. 2002;1(1):3-52.

[86] Man KF, Tang KS, Kwong S. Genetic algorithms: concepts and applications [in engineering design]. IEEE Transactions on Industrial Electronics. 1996;43(5):519-534.

[87] Ferreira C. In: Gene Expression Programming in Problem Solving. Springer London; 2002. p. $635-653$.

[88] Ye Z, Ma L, Chen H. A hybrid rice optimization algorithm. In: 2016 11th International Conference on Computer Science Education (ICCSE); 2016. p. 169-174.

[89] Cortés P, García JM, Onieva L, Muñuzuri J, Guadix J. Viral System to Solve Optimization Problems: An Immune-Inspired Computational Intelligence Approach. In: Artificial Immune Systems; 2008. p. 83-94.

[90] Tayeb FBS, Bessedik M, Benbouzid M, Cheurfi H, Blizak A. Research on permutation flow-shop scheduling problem based on improved genetic immune algorithm with vaccinated offspring. Procedia Computer Science. $2017 ; 112: 427-436$.

[91] Mehrabian AR, Lucas C. A novel numerical optimization algorithm inspired from weed colonization. Ecological Informatics. 2006;1(4):355-366.

[92] Abbass HA. MBO: marriage in honey bees optimization-a Haplometrosis polygynous swarming approach. In: Proceedings of the 2001 IEEE Congress on Evolutionary Computation. vol. 1; 2001. p. 207-214.

[93] Bidar M, Kanan HR, Mouhoub M, Sadaoui S. Mushroom Reproduction Optimization (MRO): A Novel Nature-Inspired Evolutionary Algorithm. In: 2018 IEEE Congress on Evolutionary Computation (CEC). 8477837; 2018. p. 1-10. 
[94] Sung Hoon Jung. Queen-bee evolution for genetic algorithms. Electronics Letters. 2003;39(6):575-576.

[95] Anandaraman C, Sankar AVM, Natarajan R. A new evolutionary algorithm based on bacterial evolution and its application for scheduling a flexible manufacturing system. Jurnal Teknik Industri. 2012;14(1):1-12.

[96] Taherdangkoo M, Yazdi M, Bagheri MH. Stem Cells Optimization Algorithm. In: Huang DS, Gan Y, Premaratne P, Han K, editors. Bio-Inspired Computing and Applications. Springer Berlin Heidelberg; 2012. p. 394-403.

[97] Nara K, Takeyama T, Hyungchul Kim. A new evolutionary algorithm based on sheep flocks heredity model and its application to scheduling problem. In: IEEE SMC'99 Conference Proceedings. 1999 IEEE International Conference on Systems, Man, and Cybernetics. vol. 6; 1999. p. 503-508.

[98] Pattnaik SS, Bakwad KM, Sohi BS, Ratho RK, Devi S. Swine Influenza Models Based Optimization (SIMBO). Applied Soft Computing. 2013;13(1):628 - 653 .

[99] Zelinka I. In: SOMA — Self-Organizing Migrating Algorithm. Berlin, Heidelberg: Springer Berlin Heidelberg; 2004. p. 167-217.

[100] Puris A, Bello R, Molina D, Herrera F. Variable mesh optimization for continuous optimization problems. Soft Computing. 2012;16(3):511-525.

[101] Jaderyan M, Khotanlou H. Virulence Optimization Algorithm. Applied Soft Computing. 2016;43:596 - 618.

[102] Brabazon A, McGarraghy S. Foraging-Inspired Optimisation Algorithms. Natural Computing Series, Springer; 2018.

[103] Uymaz SA, Tezel G, Yel E. Artificial algae algorithm (AAA) for nonlinear global optimization. Applied Soft Computing. 2014;31:153-171.

[104] Muñoz MA, López JA, Caicedo E. An artificial beehive algorithm for continuous optimization. International Journal of Intelligent Systems. 2009;24(11):1080-1093.

[105] Naderi B, Khalili M, Khamseh AA. Mathematical models and a hunting search algorithm for the no-wait flowshop scheduling with parallel machines. International Journal of Production Research. 2014;52(9):2667-2681.

[106] Odili JB, Mohmad Kahar MN. Solving the Traveling Salesman's Problem Using the African Buffalo Optimization. Computational Intelligence and Neuroscience. 2016;2016:1-12.

[107] Almonacid B, Soto R. Andean Condor Algorithm for cell formation problems. Natural Computing. 2019;18(2):351-381. 
[108] Lamy JB. Artificial Feeding Birds (AFB): a new metaheuristic inspired by the behavior of pigeons. In: Advances in nature-inspired computing and applications; 2019. p. 43-60.

[109] Li X, Zhang J, Yin M. Animal migration optimization: an optimization algorithm inspired by animal migration behavior. Neural Computing and Applications. 2014;24(7):1867-1877.

[110] Mirjalili S. The Ant Lion Optimizer. Advances in Engineering Software. 2015;83:80-98.

[111] Pook MF, Ramlan EI. The Anglerfish algorithm: a derivation of randomized incremental construction technique for solving the traveling salesman problem. Evolutionary Intelligence. 2019;12(1):11-20.

[112] Chen T, Pang L, Jiang Du, Liu Z, Zhang L. Artificial Searching Swarm Algorithm for solving constrained optimization problems. In: 2009 IEEE International Conference on Intelligent Computing and Intelligent Systems. vol. 1; 2009. p. $562-565$.

[113] Chen T, Wang Y, Li J. Artificial tribe algorithm and its performance analysis. Journal of Software. 2012;7(3):651-656.

[114] Subramanian C, Sekar ASS, Subramanian K. A New Engineering Optimization Method African Wild Dog Algorithm. International Journal of Soft Computing. 2013;8(3):163-170.

[115] Alsattar HA, Zaidan AA, Zaidan BB. Novel meta-heuristic bald eagle search optimisation algorithm. Artificial Intelligence Review. 2019;p. 1-28.

[116] Pham DT, Ghanbarzadeh A, Koç E, Otri S, Rahim S, Zaidi M. The Bees Algorithm - A Novel Tool for Complex Optimisation Problems. In: Intelligent Production Machines and Systems. Elsevier Science Ltd; 2006. p. 454 - 459.

[117] Comellas F, Martinez-Navarro J. Bumblebees: A Multiagent Combinatorial Optimization Algorithm Inspired by Social Insect Behaviour. In: Proceedings of the First ACM/SIGEVO Summit on Genetic and Evolutionary Computation. GEC '09; 2009. p. 811-814.

[118] Kazikova A, Pluhacek M, Senkerik R, Viktorin A. Proposal of a new swarm optimization method inspired in bison behavior. Advances in Intelligent Systems and Computing. 2019;837:146-156.

[119] Häckel S, Dippold P. The Bee Colony-inspired Algorithm (BCiA): A Two-stage Approach for Solving the Vehicle Routing Problem with Time Windows. In: Proceedings of the 11th Annual Conference on Genetic and Evolutionary Computation. GECCO '09; 2009. p. 25-32.

[120] Teodorović D, Dell'Orco M. Bee colony optimization - A cooperative learning approach to complex transportation problems. Advanced OR and AI Methods in Transportation. 2005;p. 51-60. 
[121] Niu B, Wang H. Bacterial Colony Optimization: Principles and Foundations. In: Emerging Intelligent Computing Technology and Applications; 2012. p. 501-506.

[122] Müller SD, Marchetto J, Airaghi S, Koumoutsakos P. Optimization Based on Bacterial Chemotaxis. IEEE Transactions On Evolutionary Computation. 2002;6(1):16-29.

[123] Dutta T, Bhattacharyya S, Dey S, Platos J. Border Collie Optimization. IEEE Access. 2020;8:109177-109197.

[124] Chen T, Tsai P, Chu S, Pan J. A Novel Optimization Approach: Bacterial-GA Foraging. In: Second International Conference on Innovative Computing, Informatio and Control (ICICIC 2007); 2007. p. 391-391.

[125] Wedde HF, Farooq M, Zhang Y. BeeHive: An Efficient Fault-Tolerant Routing Algorithm Inspired by Honey Bee Behavior. In: Ant Colony Optimization and Swarm Intelligence, Proceeding; 2004. p. 83-94.

[126] Bitam S, Zeadally S, Mellouk A. Fog computing job scheduling optimization based on bees swarm. Enterprise Information Systems. 2018;12(4):373-397.

[127] Malakooti B, Kim H, Sheikh S. Bat intelligence search with application to multi-objective multiprocessor scheduling optimization. International Journal of Advanced Manufacturing Technology. 2012;60(9-12):1071-1086.

[128] Zhang Q, Wang R, Yang J, Lewis A, Chiclana F, Yang S. Biology migration algorithm: a new nature-inspired heuristic methodology for global optimization. Soft Computing. 2019;23(16):7333-7358.

[129] Sulaiman MH, Mustaffa Z, Saari MM, Daniyal H, Mohamad AJ, Othman MR, et al. Barnacles Mating Optimizer Algorithm for Optimization. In: Proceedings of the 10th National Technical Seminar on Underwater System Technology 2018. Springer Singapore; 2019. p. 211-218.

[130] Taherdangkoo M, Shirzadi MH, Yazdi M, Bagher MH. A robust clustering method based on blind, naked mole-rats (BNMR) algorithm. Swarm and Evolutionary Computation. 2013;10:1-11.

[131] Kumar A, Misra RK, Singh D. Butterfly optimizer. In: 2015 IEEE Workshop on Computational Intelligence: Theories, Applications and Future Directions (WCI); 2015. p. 1-6.

[132] Das AK, Pratihar DK. A new bonobo optimizer (BO) for real-parameter optimization. In: 2019 IEEE Region 10 Symposium (TENSYMP). IEEE; 2019. p. 108-113.

[133] FINDIK O. Bull optimization algorithm based on genetic operators for continuous optimization problems. TURKISH JOURNAL OF ELECTRICAL ENGINEERING \& COMPUTER SCIENCES. 2015 01;23:2225-2239. 
[134] Sato T, Hagiwara M. Bee System: Finding solution by a concentrated search. In: Proceedings of the IEEE International Conference on Systems, Man and Cybernetics. vol. 4; 1997. p. 3954-3959.

[135] Lucic P, Teodorovic D. Transportation modeling: an artificial life approach. In: 14th IEEE International Conference on Tools with Artificial Intelligence, 2002. (ICTAI 2002). Proceedings.; 2002. p. 216-223.

[136] Meng XB, Gao XZ, Lu L, Liu Y, Zhang H. A new bio-inspired optimisation algorithm: Bird Swarm Algorithm. Journal of Experimental and Theoretical Artificial Intelligence. 2016;28(4):673-687.

[137] Akbari R, Mohammadi A, Ziarati K. A novel bee swarm optimization algorithm for numerical function optimization. Communications in Nonlinear Science and Numerical Simulation. 2010;15(10):3142-3155.

[138] de Oliveira DR, Parpinelli RS, Lopes HS. 5. In: Bioluminescent Swarm Optimization Algorithm. IntechOpen; 2011. .

[139] Drias H, Sadeg S, Yahi S. Cooperative Bees Swarm for Solving the Maximum Weighted Satisfiability Problem. In: Computational Intelligence and Bioinspired Systems; 2005. p. 318-325.

[140] Arshaghi A, Ashourian M, Ghabeli L. Buzzard optimization algorithm: a nature-inspired metaheuristic algorithm. Majlesi Journal of Electrical Engineering. 2019;13(3):83-98.

[141] Hayyolalam V, Pourhaji Kazem AA. Black Widow Optimization Algorithm: A novel meta-heuristic approach for solving engineering optimization problems. Engineering Applications of Artificial Intelligence. 2020;87(103249):1-28.

[142] K SR, Panwar L, Panigrahi BK, Kumar R. Binary whale optimization algorithm: a new metaheuristic approach for profit-based unit commitment problems in competitive electricity markets. Engineering Optimization. 2019;51(3):369-389.

[143] Cuevas E, González M, Zaldivar D, Pérez-Cisneros M, García G. An algorithm for global optimization inspired by collective animal behavior. Discrete Dynamics in Nature and Society. 2012;2012:1-24.

[144] Klein CE, Mariani VC, Coelho LDS. Cheetah based optimization algorithm: A novel swarm intelligence paradigm; 2018. p. 685-690.

[145] Shiqin Y, Jianjun J, Guangxing Y. Improved binary particle swarm optimization using catfish effect for feature selection. Expert Systems with Applications. 2011;38(10):12699-12707.

[146] Canayaz M, Karci A. Cricket behaviour-based evolutionary computation technique in solving engineering optimization problems. Applied Intelligence. 2016;44(2):362-376. 
[147] Pierezan J, Maidl G, Massashi Yamao E, dos Santos Coelho L, Cocco Mariani V. Cultural coyote optimization algorithm applied to a heavy duty gas turbine operation. Energy Conversion and Management. 2019;199.

[148] Rizk-Allah RM, Hassanien AE, Bhattacharyya S. Chaotic crow search algorithm for fractional optimization problems. Applied Soft Computing. 2018;71:1161-1175.

[149] Sayed GI, Tharwat A, Hassanien AE. Chaotic dragonfly algorithm: an improved metaheuristic algorithm for feature selection. Applied Intelligence. 2019;49(1):188-205.

[150] Eesa Sabry A, Adbulazeez Brifcani AM, Orman Z. Cuttlefish Algorithm - A Novel Bio-Inspired Optimization Algorithm. International Journal of Scientific and Engineering Research. 2013;4(9):1978-1986.

[151] Iordache S. A Hierarchical Cooperative Evolutionary Algorithm. In: Proceedings of the 12th Annual Conference on Genetic and Evolutionary Computation. GECCO ’10; 2010. p. 225-232.

[152] Al-Obaidi A, Abdullah H, Othman Z. Camel Herds Algorithm: a New Swarm Intelligent Algorithm to Solve Optimization Problems. International Journal on Perceptive and Cognitive Computing. 2017 05;3.

[153] Rajabioun R. Cuckoo Optimization Algorithm. Applied Soft Computing. 2011;11(8):5508-5518.

[154] Ibrahim MK, Salim Ali R. Novel Optimization Algorithm Inspired by Camel Traveling Behavior. Iraq Journal Electrical and Electronic Engineering. 2016;12(2):167-177.

[155] Pierezan J, Dos Santos Coelho L. Coyote Optimization Algorithm: A New Metaheuristic for Global Optimization Problems. In: 2018 IEEE Congress on Evolutionary Computation (CEC); 2018. p. 1-8.

[156] Yang X, Deb S. Cuckoo Search via Lévy flights. In: 2009 World Congress on Nature Biologically Inspired Computing (NaBIC); 2009. p. 210-214.

[157] Askarzadeh A. A novel metaheuristic method for solving constrained engineering optimization problems: Crow search algorithm. Computers and Structures. 2016;169:1-12.

[158] Chu SC, Tsai Pw, Pan JS. Cat Swarm Optimization. In: PRICAI 2006: Trends in Artificial Intelligence; 2006. p. $854-858$.

[159] Meng X, Liu Y, Gao X, Zhang H. A New Bio-inspired Algorithm: Chicken Swarm Optimization. In: Tan Y, Shi Y, Coello CAC, editors. Advances in Swarm Intelligence; 2014. p. 86-94.

[160] Bhardwaj S, Kim DS. Dragonfly-based swarm system model for node identification in ultra-reliable low-latency communication. Neural Computing and Applications. 2020;p. 1-44. 
[161] Kaveh A, Farhoudi N. A new optimization method: Dolphin echolocation. Advances in Engineering Software. 2013;59:53-70.

[162] Brammya G, Praveena S, Ninu Preetha NS, Ramya R, Rajakumar BR, Binu D. Deer Hunting Optimization Algorithm: A New Nature-Inspired Meta-heuristic Paradigm. The Computer Journal. 2019;p. 1-20.

[163] Shiqin Y, Jianjun J, Guangxing Y. A Dolphin Partner Optimization. In: 2009 WRI Global Congress on Intelligent Systems. vol. 1; 2009. p. 124-128.

[164] Dehghani M, Mardaneh M, Malik OP, NouraeiPour SM. DTO: Donkey Theorem Optimization. In: 2019 27th Iranian Conference on Electrical Engineering (ICEE); 2019. p. 1855-1859.

[165] Wang G, Deb S, d S Coelho L. Elephant Herding Optimization. In: 2015 3rd International Symposium on Computational and Business Intelligence (ISCBI); 2015. p. 1-5.

[166] Harifi S, Khalilian M, Mohammadzadeh J, Ebrahimnejad S. Emperor Penguins Colony: a new metaheuristic algorithm for optimization. Evolutionary Intelligence. 2019 02;.

[167] Dhiman G, Kumar V. Emperor penguin optimizer: a bio-inspired algorithm for engineering problems. Knowledge-Based Systems. 2018;159:20-50.

[168] Yang XS, Deb S. In: Eagle Strategy Using Lévy Walk and Firefly Algorithms for Stochastic Optimization. Berlin, Heidelberg: Springer Berlin Heidelberg; 2010. p. 101-111.

[169] Deb S, Fong S, Tian Z. Elephant Search Algorithm for Optimization Problems. The Tenth International Conference on Digital Information Management. 2015;p. 249-255.

[170] Mandal S. Elephant swarm water search algorithm for global optimization. Sādhanā. 2018 02;43.

[171] Sur C, Sharma S, Shukla A. Egyptian Vulture Optimization Algorithm - A New Nature Inspired Meta-heuristics for Knapsack Problem. In: The 9th International Conference on Computing and InformationTechnology (IC2IT2013); 2013. p. 227-237.

[172] Cui X, Gao J, Potok TE. A flocking based algorithm for document clustering analysis. Journal of Systems Architecture. 2006;52(8-9):505-515.

[173] Chu Y, Mi H, Liao H, Ji Z, Wu QH. A Fast Bacterial Swarming Algorithm for high-dimensional function optimization. In: 2008 IEEE Congress on Evolutionary Computation (IEEE World Congress on Computational Intelligence); 2008. p. 3135-3140. 
[174] Mutazono A, Sugano M, Murata M. Frog call-inspired self-organizing anti-phase synchronization for wireless sensor networks. In: 2009 2nd International Workshop on Nonlinear Dynamics and Synchronization; 2009. p. 81-88.

[175] Bellaachia A, Bari A. Flock by Leader: A Novel Machine Learning Biologically Inspired Clustering Algorithm. In: Advances in Swarm Intelligence. Berlin, Heidelberg: Springer Berlin Heidelberg; 2012. p. 117-126.

[176] Pan WT. A new Fruit Fly Optimization Algorithm: Taking the financial distress model as an example. Knowledge-Based Systems. 2012;26:69-74.

[177] de Vasconcelos Segundo EH, Mariani VC, dos Santos Coelho L. Design of heat exchangers using Falcon Optimization Algorithm. Applied Thermal Engineering. 2019;156:119-144.

[178] Li XL, Shao ZJ, Qian JX. Optimizing method based on autonomous animats: Fish-swarm Algorithm. Xitong Gongcheng Lilun yu Shijian/System Engineering Theory and Practice. 2002;22(11):32.

[179] Tsai HC, Lin YH. Modification of the fish swarm algorithm with particle swarm optimization formulation and communication behavior. Applied Soft Computing. 2011;11(8):5367 - 5374.

[180] Bastos Filho CJA, de Lima Neto FB, Lins AJCC, Nascimento AIS, Lima MP. A novel search algorithm based on fish school behavior. In: 2008 IEEE International Conference on Systems, Man and Cybernetics; 2008. p. $2646-2651$.

[181] Min H, Wang Z. Design and analysis of Group Escape Behavior for distributed autonomous mobile robots. In: 2011 IEEE International Conference on Robotics and Automation; 2011. p. 6128-6135.

[182] Su S, Wang J, Fan W, Yin X. Good Lattice Swarm Algorithm for Constrained Engineering Design Optimization. In: 2007 International Conference on Wireless Communications, Networking and Mobile Computing; 2007. p. $6421-6424$.

[183] He S, Wu QH, Saunders JR. Group Search Optimizer: An Optimization Algorithm Inspired by Animal Searching Behavior. IEEE Transactions on Evolutionary Computation. 2009;13(5):973-990.

[184] Wang J, Wang D. Particle swarm optimization with a leader and followers. Progress in Natural Science. $2008 ; 18(11): 1437-1443$.

[185] Mirjalili S, Mirjalili SM, Lewis A. Grey Wolf Optimizer. Advances in Engineering Software. 2014;69:46-61.

[186] Morais RG, Nedjah N, Mourelle LM. A novel metaheuristic inspired by Hitchcock birds' behavior for efficient optimization of large search spaces of high dimensionality. Soft Computing. 2020;24(8):5633-5655.

[187] Bozorg-Haddad O, Afshar A, Mariño M. Honey-Bees Mating Optimization (HBMO) Algorithm: A New Heuristic Approach for Water Resources Optimization. Water Resources Management. 2006 01;20:661-680. 
[188] Heidari AA, Mirjalili S, Faris H, Aljarah I, Mafarja M, Chen H. Harris hawks optimization: Algorithm and applications. Future Generation Computer Systems. 2019;97:849 - 872.

[189] El-Dosuky M, El-Bassiouny A, Hamza T, Rashad M. New Hoopoe Heuristic Optimization. International Journal of Science and Advanced Technology. 2012;2(9):85-90.

[190] Moldovan D. Horse Optimization Algorithm: A Novel Bio-Inspired Algorithm for Solving Global Optimization Problems. In: Artificial Intelligence and Bioinspired Computational Methods. Springer International Publishing; 2020. p. 195-209.

[191] Oftadeh R, Mahjoob MJ, Shariatpanahi M. A novel meta-heuristic optimization algorithm inspired by group hunting of animals: Hunting search. Computers and Mathematics with Applications. 2010;60(7):2087-2098.

[192] Quijano N, Passino KM. Honey Bee Social Foraging Algorithms for Resource Allocation, Part I: Algorithm and Theory. In: 2007 American Control Conference; 2007. p. 3383-3388.

[193] Chen H, Zhu Y, Hu K, He X. Hierarchical Swarm Model: A New Approach to Optimization. Discrete Dynamics in Nature and Society. 2010;2010:1-30.

[194] Ali A, Zafar K, Bakhshi T. On Nature-Inspired Dynamic Route Planning: Hammerhead Shark Optimization Algorithm. In: 2019 15th International Conference on Emerging Technologies (ICET); 2019. p. 1-6.

[195] Maciel O, Valdivia A, Oliva D, Cuevas E, Zaldívar D, Pérez-Cisneros M. A novel hybrid metaheuristic optimization method: hypercube natural aggregation algorithm. Soft Computing. 2019 Oct;

[196] Torabi S, Safi-Esfahani F. Improved Raven Roosting Optimization algorithm (IRRO). Swarm and Evolutionary Computation. 2018;40:144 - 154 .

[197] Tang D, Dong S, Jiang Y, Li H, Huang Y. ITGO: Invasive tumor growth optimization algorithm. Applied Soft Computing. 2015;36:670-698.

[198] Chen C, Tsai Y, Liu I, Lai C, Yeh Y, Kuo S, et al. A Novel Metaheuristic: Jaguar Algorithm with Learning Behavior. In: 2015 IEEE International Conference on Systems, Man, and Cybernetics; 2015. p. 1595-1600.

[199] Hernández H, Blum C. Distributed graph coloring: an approach based on the calling behavior of Japanese tree frogs. Swarm Intelligence. 2012;6(2):117-150.

[200] Agbehadji IE, Millham R, Fong S. Kestrel-Based Search Algorithm for Association Rule Mining and Classification of Frequently Changed Items. In: 2016 8th International Conference on Computational Intelligence and Communication Networks (CICN); 2016. p. 356-360. 
[201] Biyanto TR, Irawan S, Febrianto HY, Afdanny N, Rahman AH, Gunawan KS, et al. Killer whale algorithm: an algorithm inspired by the life of killer whale. Procedia computer science. 2017;124:151-157.

[202] Rajakumar BR. The Lion's Algorithm: A New Nature-Inspired Search Algorithm. Procedia Technology. 2012;6:126-135.

[203] Wang P, Zhu Z, Huang S. Seven-Spot Ladybird Optimization: A Novel and Efficient Metaheuristic Algorithm for Numerical Optimization. The Scientific World Journal. 2013;2013:1-11.

[204] Hosseini E. Laying chicken algorithm: A new meta-heuristic approach to solve continuous programming problems. J Appl Comput Math. 2017;6(1):344-351.

[205] Yazdani M, Jolai F. Lion Optimization Algorithm (LOA): A nature-inspired metaheuristic algorithm. Journal of Computational Design and Engineering. 2016;3(1):24-36.

[206] Wang B, Jin X, Cheng B. Lion pride optimizer: An optimization algorithm inspired by lion pride behavior. Science China Information Sciences. 2012;55(10):2369-2389.

[207] Chen S. An Analysis of Locust Swarms on Large Scale Global Optimization Problems. In: Artificial Life: Borrowing from Biology; 2009. p. 211-220.

[208] Cuevas E, Gonzalez A, Zaldivar D, Cisneros M. An optimisation algorithm based on the behaviour of locust swarms. International Journal of Bio-Inspired Computation. 2015 01;7:402-407.

[209] Zervoudakis K, Tsafarakis S. A mayfly optimization algorithm. Computers \& Industrial Engineering. 2020;145(106559):1-23.

[210] Mo H, Xu L. Magnetotactic bacteria optimization algorithm for multimodal optimization. In: 2013 IEEE Symposium on Swarm Intelligence (SIS); 2013. p. 240-247.

[211] Wang GG, Deb S, Cui Z. Monarch butterfly optimization. Neural Computing and Applications. 2015;p. 1-20.

[212] Duman E, Uysal M, Alkaya AF. Migrating Birds Optimization: A new metaheuristic approach and its performance on quadratic assignment problem. Information Sciences. 2012;217:65-77.

[213] Jahani E, Chizari M. Tackling global optimization problems with a novel algorithm - Mouth Brooding Fish algorithm. Applied Soft Computing Journal. 2018;62:987-1002.

[214] Walton S, Hassan O, Morgan K, Brown MR. Modified cuckoo search: A new gradient free optimisation algorithm. Chaos, Solitons and Fractals. 2011;44(9):710-718. 
[215] Obagbuwa IC, Adewumi AO. An Improved Cockroach Swarm Optimization. ScientificWorld Journal. 2014;p. 1-13.

[216] Mirjalili S. Moth-flame optimization algorithm: A novel nature-inspired heuristic paradigm. Knowledge-Based Systems. 2015;89:228-249.

[217] Alauddin M. Mosquito flying optimization (MFO). In: 2016 International Conference on Electrical, Electronics, and Optimization Techniques (ICEEOT). IEEE; 2016. p. 79-84.

[218] Klein CE, Coelho LDS. Meerkats-inspired algorithm for global optimization problems; 2018. p. 679-684.

[219] ul Amir Afsar Minhas F, Arif M. MOX: A novel global optimization algorithm inspired from Oviposition site selection and egg hatching inhibition in mosquitoes. Applied Soft Computing. 2011;11(8):4614 - 4625.

[220] Faramarzi A, Heidarinejad M, Mirjalili S, Gandomi AH. Marine Predators Algorithm: A nature-inspired metaheuristic. Expert Systems with Applications. 2020;152(113377):1-28.

[221] Mucherino A, Seref O. Monkey search: a novel metaheuristic search for global optimization. In: American Institute of Physics. vol. 953; 2007. p. 162-173.

[222] Luo F, Zhao J, Dong ZY. A new metaheuristic algorithm for real-parameter optimization: Natural aggregation algorithm. In: 2016 IEEE Congress on Evolutionary Computation (CEC); 2016. p. 94-103.

[223] Salgotra R, Singh U. The naked mole-rat algorithm. Neural Computing and Applications. 2019 Sep;.

[224] Salih SQ, Alsewari AA. A new algorithm for normal and large-scale optimization problems: Nomadic People Optimizer. Neural Computing and Applications. 2019 Oct;.

[225] Drias H, Drias Y, Khennak I. A New Swarm Algorithm Based on Orcas Intelligence for Solving Maze Problems. In: Trends and Innovations in Information Systems and Technologies. Springer International Publishing; 2020. p. 788-797.

[226] Maia RD, d Castro LN, Caminhas WM. OptBees - A Bee-Inspired Algorithm for Solving Continuous Optimization Problems. In: 2013 BRICS Congress on Computational Intelligence and 11th Brazilian Congress on Computational Intelligence; 2013. p. 142-151.

[227] Zhu GY, Zhang WB. Optimal foraging algorithm for global optimization. Applied Soft Computing. 2017;51:294 313.

[228] de Vasconcelos Segundo EH, Mariani VC, dos Santos Coelho L. Metaheuristic inspired on owls behavior applied to heat exchangers design. Thermal Science and Engineering Progress. 2019;14:100431. 
[229] Kallioras NA, Lagaros ND, Avtzis DN. Pity beetle algorithm - A new metaheuristic inspired by the behavior of bark beetles. Advances in Engineering Software. 2018;121:147-166.

[230] Połap D, Wozniak M. Polar Bear Optimization Algorithm: Meta-Heuristic with Fast Population Movement and Dynamic Birth and Death Mechanism. Symmetry. 2017;9(203):1-20.

[231] Duan H, Qiao P. Pigeon-inspired optimization: a new swarm intelligence optimizer for air robot path planning. International Journal of Intelligent Computing and Cybernetics. 2014;7(1):24-37.

[232] Zhang W, Luo Q, Zhou Y. A Method for Training RBF Neural Networks Based on Population Migration Algorithm. In: Proceedings of the 2009 International Conference on Artificial Intelligence and Computational Intelligence - Volume 01. AICI '09; 2009. p. 165-169.

[233] Tilahun SL, Choon Ong H. Prey-predator algorithm: A new metaheuristic algorithm for optimization problems. International Journal of Information Technology and Decision Making. 2015;14(6):1331-1352.

[234] Gheraibia Y, Moussaoui A. Penguins Search Optimization Algorithm (PeSOA). In: Recent Trends in Applied Artificial Intelligence; 2013. p. 222-231.

[235] Arora S, Singh S. Butterfly optimization algorithm: a novel approach for global optimization. Soft Computing. 2019;23(3):715-734.

[236] Fard AF, Hajiaghaei-Keshteli M. Red Deer Algorithm (RDA); a new optimization algorithm inspired by Red Deers' mating. In: International Conference on Industrial Engineering, IEEE.,(2016 e); 2016. p. 33-34.

[237] Połap D, Woźniak M. Red fox optimization algorithm. Expert Systems with Applications. 2021;166:114107.

[238] Wang GG, Gao XZ, Zenger K, Coelho LdS. A novel metaheuristic algorithm inspired by rhino herd behavior. In: Proceedings of The 9th EUROSIM Congress on Modelling and Simulation, EUROSIM 2016, The 57th SIMS Conference on Simulation and Modelling SIMS 2016. 142. Linköping University Electronic Press; 2018. p. $1026-1033$.

[239] Havens T, J Spain C, G Salmon N, M Keller J. Roach Infestation Optimization. In: 2008 IEEE Swarm Intelligence Symposium, SIS 2008; 2008. p. 1-7.

[240] Zangbari Koohi S, Abdul Hamid NAW, Othman M, Ibragimov G. Raccoon Optimization Algorithm. IEEE Access. 2019;7:5383-5399.

[241] Sharma A. A new optimizing algorithm using reincarnation concept. In: 2010 11th International Symposium on Computational Intelligence and Informatics (CINTI); 2010. p. 281-288. 
[242] Brabazon A, Cui W, O’neill M. The Raven Roosting Optimisation Algorithm. Soft Computing. 2016;20(2):525-545.

[243] Saadi Y, Tri I, Yanto I, Herawan T, Balakrishnan V, Chiroma H, et al. Ringed Seal Search for Global Optimization via a Sensitive Search Model. PLoS ONE. 2015 11;11.

[244] Hersovici M, Jacovi M, Maarek YS, Pelleg D, Shtalhaim M, Ur S. The shark-search algorithm. An application: tailored Web site mapping. Computer Networks and ISDN Systems. 1998;30(1):317-326.

[245] McCaffrey JD. Generation of pairwise test sets using a simulated bee colony algorithm. In: 2009 IEEE International Conference on Information Reuse Integration; 2009. p. 115-119.

[246] Samareh Moosavi SH, Khatibi Bardsiri V. Satin bowerbird optimizer: A new optimization algorithm to optimize ANFIS for software development effort estimation. Engineering Applications of Artificial Intelligence. 2017;60:1-15.

[247] Mirjalili S. SCA: A Sine Cosine Algorithm for solving optimization problems. Knowledge-Based Systems. 2016;96:120-133.

[248] Rakhshani H, Rahati A. Snap-drift cuckoo search: A novel cuckoo search optimization algorithm. Applied Soft Computing. 2017;52:771- 794 .

[249] Eusuff M, Lansey K, Pasha F. Shuffled frog-leaping algorithm: a memetic metaheuristic for discrete optimization. Engineering Optimization. 2006;38(2):129-154.

[250] Dhiman G, Kumar V. Spotted hyena optimizer: a novel bio-inspired based metaheuristic technique for engineering applications. Advances in Engineering Software. 2017;114:48-70.

[251] Fausto F, Cuevas E, Valdivia A, González A. A global optimization algorithm inspired in the behavior of selfish herds. Biosystems. 2017;160:39-55.

[252] Su MC, Su SY, Zhao YX. A swarm-inspired projection algorithm. Pattern Recognition. 2009;42(11):2764-2786.

[253] Monismith DR, Mayfield BE. Slime Mold as a model for numerical optimization. In: 2008 IEEE Swarm Intelligence Symposium; 2008. p. 1-8.

[254] Raouf O, M Hezam I. Sperm motility algorithm: a novel metaheuristic approach for global optimisation. International Journal of Operational Research. 2017 01;28:143.

[255] Chand Bansal J, Sharma H, Singh Jadon S, Clerc M. Spider Monkey Optimization algorithm for numerical optimization. Memetic Computation. 2014;6:31-47. 
[256] Dai C, Zhu Y, Chen W. Seeker Optimization Algorithm. In: Computational Intelligence and Security; 2007. p. 167-176.

[257] Dhiman G, Kumar V. Seagull optimization algorithm: Theory and its applications for large-scale industrial engineering problems. Knowledge-Based Systems. 2019;165:169-196.

[258] Kaur A, Jain S, Goel S. Sandpiper optimization algorithm: a novel approach for solving real-life engineering problems. Applied Intelligence. 2020;50(2):582-619.

[259] Shadravan S, Naji HR, Bardsiri VK. The Sailfish Optimizer: A novel nature-inspired metaheuristic algorithm for solving constrained engineering optimization problems. Engineering Applications of Artificial Intelligence. 2019;80:20-34.

[260] Cheng MY, Prayogo D. Symbiotic Organisms Search: A new metaheuristic optimization algorithm. Computers and Structures. 2014;139:98-112.

[261] Dhiman G, Kaur A. STOA: A bio-inspired based optimization algorithm for industrial engineering problems. Engineering Applications of Artificial Intelligence. 2019;82:148-174.

[262] Yu JJQ, Li VOK. A social spider algorithm for global optimization. Applied Soft Computing. 2015;30:614-627.

[263] Jain M, Singh V, Rani A. A novel nature-inspired algorithm for optimization: Squirrel search algorithm. Swarm and Evolutionary Computation. 2019;44:148 - 175.

[264] Mirjalili S, Gandomi AH, Mirjalili SZ, Saremi S, Faris H, Mirjalili SM. Salp Swarm Algorithm: A bio-inspired optimizer for engineering design problems. Advances in Engineering Software. 2017;114:163 - 191.

[265] Abedinia O, Amjady N, Ghasemi A. A New Metaheuristic Algorithm Based on Shark Smell Optimization. Complexity. 2016;21(5):97-116.

[266] Neshat M, Sepidnam G, Sargolzaei M. Swallow swarm optimization algorithm: a new method to optimization. Neural Computing and Applications. 2013;23(2):429-454.

[267] Cuevas E, Cienfuegos M, Záldivar D, Pérez-Cisneros M. A swarm optimization algorithm inspired in the behavior of the social-spider. Expert Systems with Applications. 2013;40(16):6374-6384.

[268] Shehadeh H, Idris M, Ahmedy I, Ramli R, N M N. The Multi-Objective Optimization Algorithm Based on Sperm Fertilization Procedure (MOSFP) Method for Solving Wireless Sensor Networks Optimization Problems in Smart Grid Applications. Energies. 2018 01;11:97. 
[269] Omidvar R, Parvin H, Rad F. SSPCO Optimization Algorithm (See-See Partridge Chicks Optimization). In: 2015 Fourteenth Mexican International Conference on Artificial Intelligence (MICAI); 2015. p. 101-106.

[270] Haiyan Q, Xinling S. A Surface-Simplex Swarm Evolution Algorithm. Advances in Engineering Software. $2017 ; 22(1): 38-50$.

[271] Ebrahimi A, Khamehchi E. Sperm whale algorithm: An effective metaheuristic algorithm for production optimization problems. Journal of Natural Gas Science and Engineering. 2016;29:211-222.

[272] Zungeru AM, Ang LM, Seng KP. Termite-hill: Performance optimized swarm intelligence based routing algorithm for wireless sensor networks. Journal of Network and Computer Applications. 2012;35(6):1901-1917.

[273] Hedayatzadeh R, Akhavan Salmassi F, Keshtgari M, Akbari R, Ziarati K. Termite colony optimization: A novel approach for optimizing continuous problems. In: 2010 18th Iranian Conference on Electrical Engineering; 2010. p. $553-558$.

[274] Mozaffari A, Goudarzi AM, Fathi A. Bio-inspired methods for fast and robust arrangement of thermoelectric modulus. International Journal of Bio-Inspired Computation (IJBIC). 2013;5(1):19-34.

[275] Kaur S, Awasthi LK, Sangal AL, Dhiman G. Tunicate Swarm Algorithm: A new bio-inspired based metaheuristic paradigm for global optimization. Engineering Applications of Artificial Intelligence. 2020;90(103541):1-29.

[276] Yang X, Lees JM, Morley CT. Application of Virtual Ant Algorithms in the Optimization of CFRP Shear Strengthened Precracked Structures. In: Computational Science - ICCS 2006, 6th International Conference, Proceedings, Part I; 2006. p. 834-837.

[277] Yang XS. Engineering Optimizations via Nature-Inspired Virtual Bee Algorithms. In: Mira J, Álvarez JR, editors. Artificial Intelligence and Knowledge Engineering Applications: A Bioinspired Approach. Springer; 2005. p. 317-323.

[278] Li MD, Zhao H, Weng XW, Han T. A novel nature-inspired algorithm for optimization: Virus colony search. Advances in Engineering Software. 2016;92:65-88.

[279] Juarez JRC, Wang HJ, Lai YC, Liang YC. Virus Optimization Algorithm (VOA): A novel metaheuristic for solving continuous optimization problems. In: Proceedings of the 2009 Asia Pacific Industrial Engineering and Management Systems Conference (APIEMS 2009); 2009. p. 2166-2174.

[280] Cortés P, García JM, Muñuzuri J, Onieva L. Viral systems: A new bio-inspired optimisation approach. Computers and Operations Research. 2008;35:2840-2860. 
[281] Liu CY, Yan XH, Wu H. The Wolf Colony Algorithm and Its Application. Chinese Journal of Electronics. 2011;20:212-216.

[282] Arnaout JP. Worm Optimization: A novel optimization algorithm inspired by C. Elegans. In: Proceedings of the 2014 International Conference on Industrial Engineering and Operations Management; 2014. p. 2499-2505.

[283] Yang C, Tu X, Chen J. Algorithm of Marriage in Honey Bees Optimization Based on the Wolf Pack Search. In: Proceedings of the The 2007 International Conference on Intelligent Pervasive Computing; 2007. p. $462-467$.

[284] Ting TO, Man KL, Guan SU, Nayel M, Wan K. Weightless Swarm Algorithm (WSA) for Dynamic Optimization Problems. In: Park JJ, Zomaya A, Yeo SS, Sahni S, editors. Network and Parallel Computing, IFIP International Conference on Network and Parallel Computing; 2012. p. 508-515.

[285] Tang R, Fong S, Yang XS, Deb S. Wolf search algorithm with ephemeral memory. In: Seventh International Conference on Digital Information Management (ICDIM 2012); 2012. p. 165-172.

[286] Pinto P, Runkler TA, Sousa JM. Wasp swarm optimization of logistic systems. In: Adaptive and Natural Computing Algorithms; 2005. p. 264-267.

[287] Zaldívar D, Morales B, Rodríguez A, Valdivia-G A, Cuevas E, Pérez-Cisneros M. A novel bio-inspired optimization model based on Yellow Saddle Goatfish behavior. Biosystems. 2018;174:1-21.

[288] Nguyen HT, Bhanu B. Zombie Survival Optimization: A Swarm Intelligence Algorithm Inspired By Zombie Foraging. In: 21st International Conference on Pattern Recognition (ICPR 2012); 2012. p. 987-990.

[289] Yang XS, Deb S, Mishra SK. Multi-species Cuckoo Search Algorithm for Global Optimization. Cognitive Computation. 2018 Dec;10(6):1085-1095.

[290] Hatamlou A. Black hole: A new heuristic optimization approach for data clustering. Information Sciences. $2013 ; 222: 175-184$.

[291] Yadav A, Yadav A. AEFA: Artificial electric field algorithm for global optimization. Swarm and Evolutionary Computation. 2019;48:93 - 108 .

[292] Xie L, Zeng J, Cui Z. General framework of Artificial Physics Optimization Algorithm. In: 2009 World Congress on Nature Biologically Inspired Computing (NaBIC); 2009. p. 1321-1326.

[293] Zhao W, Wang L, Zhang Z. Atom search optimization and its application to solve a hydrogeologic parameter estimation problem. Knowledge-Based Systems. 2019;163:283-304. 
[294] Erol OK, Eksin I. A new optimization method: Big Bang-Big Crunch. Advances in Engineering Software. 2006;37(2):106-111.

[295] Kaveh A, Mahdavi VR. Colliding bodies optimization: A novel meta-heuristic method. Computers and Structures. 2014;139:18-27.

[296] Feng X, Ma M, Yu H. Crystal Energy Optimization Algorithm. Computational Intelligence. 2016;32(2):284-322.

[297] Formato RA. In: Central Force Optimization: A New Nature Inspired Computational Framework for Multidimensional Search and Optimization. Springer Berlin Heidelberg; 2008. p. 221-238.

[298] Kaveh A, Talatahari S. A novel heuristic optimization method: charged system search. Acta Mechanica. 2010;213(3-4):267-289.

[299] Khalafallah A, Abdel-Raheem M. Electimize: new evolutionary algorithm for optimization with application in construction engineering. Journal of computing in civil engineering. 2011;25(3):192-201.

[300] Rahmanzadeh S, Pishvaee MS. Electron radar search algorithm: a novel developed meta-heuristic algorithm. Soft Computing. 2020;24(11):8443-8465.

[301] Kundu S. Gravitational clustering: A new approach based on the spatial distribution of the points. Pattern Recognition. 1999;32(7):1149-1160.

[302] Barzegar B, Rahmani AM, Far KZ. Gravitational emulation local search algorithm for advanced reservation and scheduling in grid systems. In: 2009 First Asian Himalayas International Conference on Internet; 2009. p. 1-5.

[303] Zheng M, Liu Gx, Zhou Cg, Liang Yc, Wang Y. Gravitation field algorithm and its application in gene cluster. Algorithms for Molecular Biology. 2010;5(1):1-32.

[304] Flores JJ, López R, Barrera J. Gravitational Interactions Optimization. In: Learning and Intelligent Optimization; 2011. p. 226-237.

[305] Beiranvand H, Rokrok E. General Relativity Search Algorithm: A Global Optimization Approach. International Journal of Computational Intelligence and Applications. 2015;14(3):1-29.

[306] Muthiah-Nakarajan V, Noel MM. Galactic Swarm Optimization: A new global optimization metaheuristic inspired by galactic motion. Applied Soft Computing. 2016;38:771-787.

[307] Wedyan A, Whalley J, Narayanan A. Hydrological Cycle Algorithm for Continuous Optimization Problems. Journal of Optimization. 2017 12;2017:1-25. 
[308] Cui Y, Guo R, Guo D. Lambda algorithm. Journal of Uncertain Systems. 2010;4(1):22-33.

[309] Zaránd G, Pázmándi F, Pál KF, Zimányi GT. Using Hysteresis for Optimization. Physical Review Letters. 2002;89:150201.

[310] Rbouh I, El Imrani AA. Hurricane-based Optimization Algorithm. AASRI Procedia. 2014;6:26 - 33.

[311] Askari H, Zahiri SH. Intelligent Gravitational Search Algorithm for optimum design of fuzzy classifier; 2012. p. 98-104.

[312] Shah-Hosseini H. The intelligent water drops algorithm: a nature-inspired swarm-based optimization algorithm. Int J Bio-Inspired Computation. 2009;1(1):71-79.

[313] Jihong Shen, Jialian Li. The principle analysis of Light Ray Optimization Algorithm. In: 2010 Second International Conference on Computational Intelligence and Natural Computing. vol. 2; 2010. p. 154-157.

[314] Shareef H, Ibrahim AA, Mutlag AH. Lightning search algorithm. Applied Soft Computing. 2015;36:315-333.

[315] Tayarani-N MH, Akbarzadeh-T MR. Magnetic Optimization Algorithms a new synthesis. In: 2008 IEEE Congress on Evolutionary Computation (IEEE World Congress on Computational Intelligence); 2008. p. 2659-2664.

[316] Mora-Gutiérrez RA, Ramírez-Rodríguez J, Rincón-García EA. An optimization algorithm inspired by musical composition. Artificial Intelligence Review. 2014;41(3):301-315.

[317] Ashrafi SM, Dariane AB. A novel and effective algorithm for numerical optimization: Melody Search (MS). In: 2011 11th International Conference on Hybrid Intelligent Systems (HIS); 2011. p. 109-114.

[318] Mirjalili S, Mirjalili SM, Hatamlou A. Multi-Verse Optimizer: a nature-inspired algorithm for global optimization. Neural Computing and Applications. 2016;27(2):495-513.

[319] Kashan AH. A new metaheuristic for optimization: Optics inspired optimization (OIO). Computers and Operations Research. 2015;55:99-125.

[320] Sacco WF, Filho HA, De Oliveira CRE. A populational particle collision algorithm applied to a nuclear reactor core design optimization. In: Joint International Topical Meeting on Mathematics and Computations and Supercomputing in Nuclear Applications, M and C + SNA 2007; 2007. p. 1-10.

[321] Taillard ÉD, Voss S. In: Popmusic - Partial Optimization Metaheuristic under Special Intensification Conditions. Springer US; 2002. p. 613-629. 
[322] Saire JEC, Túpac VYJ. An approach to real-coded quantum inspired evolutionary algorithm using particles filter. In: 2015 Latin America Congress on Computational Intelligence (LA-CCI). IEEE; 2015. p. 1-6.

[323] Kaboli SHA, Selvaraj J, Rahim NA. Rain-fall optimization algorithm: A population based algorithm for solving constrained optimization problems. Journal of Computational Science. 2017;19:31 - 42.

[324] Biyanto TR, Matradji, Febrianto HY, Afdanny N, Rahman AH, Gunawan KS. Rain Water Algorithm: Newton's Law of Rain Water Movements during Free Fall and Uniformly Accelerated Motion Utilization. AIP Conference Proceedings. 2019 Mar;2088(1):020053.

[325] Rabanal P, Rodríguez I, Rubio F. Using River Formation Dynamics to Design Heuristic Algorithms. In: Unconventional Computation; 2007. p. 163-177.

[326] Rahmani R, Yusof R. A new simple, fast and efficient algorithm for global optimization over continuous search-space problems: Radial Movement Optimization. Applied Mathematics and Computation. 2014;248:287-300.

[327] Kaveh A, Khayatazad M. A new meta-heuristic method: Ray Optimization. Computers and Structures. 2012;112-113:283-294.

[328] Hsiao YT, Chuang CL, Jiang JA, Chien CC. A novel optimization algorithm: space gravitational optimization. In: 2005 IEEE International Conference on Systems, Man and Cybernetics. vol. 3; 2005. p. 2323-2328.

[329] Tzanetos A, Dounias G. A new metaheuristic method for optimization: sonar inspired optimization. In: International Conference on Engineering Applications of Neural Networks. Springer; 2017. p. 417-428.

[330] Cuevas E, Echavarría A, Ramírez-Ortegón MA. An optimization algorithm inspired by the States of Matter that improves the balance between exploration and exploitation. Applied Intelligence. 2014;40:256-272.

[331] Tamura K, Yasuda K. Primary Study of Spiral Dynamics Inspired Optimization. IEEJ Transactions On Electrical And Electronic Engineering. 2011;6:98-100.

[332] Jin GG, Tran TD. A nature-inspired evolutionary algorithm based on spiral movements. In: Proceedings of SICE Annual Conference 2010; 2010. p. 1643-1647.

[333] Vicsek T, Czirók A, Ben-Jacob E, Cohen I, Shochet O. Novel Type of Phase Transition in a System of Self-Driven Particles. Physical Review Letters. 1995;75(6):1226-1229.

[334] Ghasemi M, Davoudkhani IF, Akbari E, Rahimnejad A, Ghavidel S, Li L. A novel and effective optimization algorithm for global optimization and its engineering applications: Turbulent Flow of Water-based Optimization (TFWO). Engineering Applications of Artificial Intelligence. 2020;92:103666. 
[335] Kaveh A, Ilchi Ghazaan M. Vibrating particles system algorithm for truss optimization with multiple natural frequency constraints. Acta Mechanica. 2017;228(1):307-322.

[336] Dogan B, Ölmez T. A new metaheuristic for numerical function optimization: Vortex Search algorithm. Information Sciences. 2015;293:125-145.

[337] Eskandar H, Sadollah A, Bahreininejad A, Hamdi M. Water cycle algorithm - A novel metaheuristic optimization method for solving constrained engineering optimization problems. Computers and Structures. 2012;110-111:151-166.

[338] Kaveh A, Bakhshpoori T. Water Evaporation Optimization: A novel physically inspired optimization algorithm. Computers and Structures. 2016;167:69-85.

[339] Yang FC, Wang YP. Water flow-like algorithm for object grouping problems. Journal of the Chinese Institute of Industrial Engineers. 2007;24(6):475-488.

[340] Basu S, Chaudhuri C, Kundu M, Nasipuri M, Basu DK. Text line extraction from multi-skewed handwritten documents. Pattern Recognition. 2007;40(6):1825-1839.

[341] Tran TH, Ng KM. A water-flow algorithm for flexible flow shop scheduling with intermediate buffers. Journal of Scheduling. 2011;14(5):483-500.

[342] Zheng YJ. Water wave optimization: A new nature-inspired metaheuristic. Computers and Operations Research. 2015;55:1-11.

[343] Irizarry R. A generalized framework for solving dynamic optimization problems using the artificial chemical process paradigm: Applications to particulate processes and discrete dynamic systems. Chemical Engineering Science. 2005;60(21):5663-5681.

[344] Alatas B. ACROA: Artificial Chemical Reaction Optimization Algorithm for global optimization. Expert Systems with Applications. 2011;38:13170-13180.

[345] Melin P, Astudillo L, Castillo O, Valdez F, Garcia M. Optimal design of type-2 and type-1 fuzzy tracking controllers for autonomous mobile robots under perturbed torques using a new chemical optimization paradigm. Expert Systems with Applications. 2013;40(8):3185-3195.

[346] Lam AYS, Li VOK. Chemical-Reaction-Inspired Metaheuristic for Optimization. IEEE Transactions on Evolutionary Computation. 2010;14(3):381-399. 
[347] Patel VK, Savsani VJ. Heat transfer search (HTS): a novel optimization algorithm. Information Science. 2015;324:217-246.

[348] Javidy B, Hatamlou A, Mirjalili S. Ions motion algorithm for solving optimization problems. Applied Soft Computing. $2015 ; 32: 72-79$.

[349] Chuang CL, Jiang JA. Integrated radiation optimization: inspired by the gravitational radiation in the curvature of space-time. In: 2007 IEEE Congress on Evolutionary Computation; 2007. p. 3157-3164.

[350] Moein S, Logeswaran R. KGMO: A swarm optimization algorithm based on the kinetic energy of gas molecules. Information Sciences. 2014;275:127-144.

[351] Murase H. Finite element inverse analysis using a photosynthetic algorithm. Computers and Electronics in Agriculture. 2000;29(1-2):115-123.

[352] Subashini P, Dhivyaprabha TT, Krishnaveni M. Synergistic Fibroblast Optimization. In: Artificial Intelligence and Evolutionary Computations in Engineering Systems; 2017. p. 285-294.

[353] Kaveh A, Dadras A. A novel meta-heuristic optimization algorithm: Thermal exchange optimization. Advances in Engineering Software. 2017;110:69-84.

[354] Huan TT, Kulkarni AJ, Kanesan J, Huang CJ, Abraham A. Ideology algorithm: a socio-inspired optimization methodology. Neural Computing and Applications. 2017;28(1):845-876.

[355] El-Abd M. Global-best brain storm optimization algorithm. Swarm and Evolutionary Computation. 2017;37:27 - 44.

[356] Bogar E, Beyhan S. Adolescent Identity Search Algorithm (AISA): A novel metaheuristic approach for solving optimization problems. Applied Soft Computing. 2020;95:106503.

[357] Bodaghi M, Samieefar K. Meta-heuristic bus transportation algorithm. Iran Journal of Computer Science. 2019 Mar;2(1):23-32.

[358] Zhang Q, Wang R, Yang J, Ding K, Li Y, Hu J. Collective decision optimization algorithm: A new heuristic optimization method. Neurocomputing. 2017;221:123-137.

[359] Li M, Zhao H, Weng X, Han T. Cognitive behavior optimization algorithm for solving optimization problems. Applied Soft Computing. 2016;39:199-222.

[360] Sharafi Y, Khanesar MA, Teshnehlab M. COOA: Competitive optimization algorithm. Swarm and Evolutionary Computation. 2016;30:39-63. 
[361] Milani A, Santucci V. Community of scientist optimization: An autonomy oriented approach to distributed optimization. AI Communications. 2012 04;25:157-172.

[362] Xidong Jin, Reynolds RG. Using knowledge-based evolutionary computation to solve nonlinear constraint optimization problems: a cultural algorithm approach. In: Proceedings of the 1999 Congress on Evolutionary Computation-CEC99. vol. 3 ; 1999. p. 1672-1678 Vol. 3.

[363] Biyanto TR, Fibrianto HY, Nugroho G, Hatta AM, Listijorini E, Budiati T, et al. Duelist algorithm: an algorithm inspired by how duelist improve their capabilities in a duel. In: International Conference on Swarm Intelligence. Springer; 2016. p. 39-47.

[364] Emami H, Derakhshan F. Election algorithm: A new socio-politically inspired strategy. AI Communications. 2015;28(3):591-603.

[365] Fadakar E, Ebrahimi M. A new metaheuristic football game inspired algorithm. In: 2016 1st Conference on Swarm Intelligence and Evolutionary Computation (CSIEC); 2016. p. 6-11.

[366] Razmjooy N, Khalilpour M, Ramezan M. A New Meta-Heuristic Optimization Algorithm Inspired by FIFA World Cup Competitions: Theory and Its Application in PID Designing for AVR System. Journal of Control, Automation and Electrical Systems. 2016;27(4):419-440.

[367] Osaba E, Diaz F, Onieva E. Golden ball: a novel meta-heuristic to solve combinatorial optimization problems based on soccer concepts. Applied Intelligence. 2014 Jul;41(1):145-166.

[368] Eita MA, Fahmy MM. Group counseling optimization. Applied Soft Computing. 2010;22:585-604.

[369] Daskin A, Kais S. Group leaders optimization algorithm. Molecular Physics. 2011;109(5):761-772.

[370] Lenord Melvix JSM. Greedy Politics Optimization: Metaheuristic inspired by political strategies adopted during state assembly elections. In: 2014 IEEE International Advance Computing Conference (IACC); 2014. p. 1157-1162.

[371] Zhang Y, Jin Z. Group Teaching Optimization Algorithm: A Novel Metaheuristic Method for Solving Global Optimization Problems. Expert Systems with Applications. 2020;148(C).

[372] Montiel O, Castillo O, Melin P, Rodríguez Díaz A, Sepúlveda R. Human evolutionary model: A new approach to optimization. Information Sciences. 2007;177(10):2075-2098.

[373] Thammano A, Moolwong J. A new computational intelligence technique based on human group formation. Expert Systems with Applications. 2010;37(2):1628 - 1634. 
[374] Zhang LM, Dahlmann C, Zhang Y. Human-Inspired Algorithms for continuous function optimization. In: 2009 IEEE International Conference on Intelligent Computing and Intelligent Systems; 2009. p. 318-321.

[375] Ghasemian H, Ghasemian F, Vahdat-Nejad H. Human urbanization algorithm: A novel metaheuristic approach. Mathematics and Computers in Simulation. 2020;178:1-15.

[376] Srivastava A, Das DK. A new Kho-Kho optimization Algorithm: An application to solve combined emission economic dispatch and combined heat and power economic dispatch problem. Engineering Applications of Artificial Intelligence. 2020;94:103763.

[377] Khatri A, Gaba A, Rana K, Kumar V. A novel life choice-based optimizer. Soft Computing. 2020;24(12):9121-9141.

[378] Gonzalez-Fernandez Y, Chen S. Leaders and followers - A new metaheuristic to avoid the bias of accumulated information. In: 2015 IEEE Congress on Evolutionary Computation (CEC); 2015. p. 776-783.

[379] Hu TC, Kahng AB, Tsao CWA. Old Bachelor Acceptance: A New Class of Non-Monotone Threshold Accepting Methods. ORSA Journal on Computing. 1995;7(4):417-425.

[380] Zhang X, Chen W, Dai C. Application of oriented search algorithm in reactive power optimization of power system. In: 2008 Third International Conference on Electric Utility Deregulation and Restructuring and Power Technologies; 2008. p. 2856-2861.

[381] Borji A, Hamide M. A new approach to global optimization motivated by parliamentary political competitions. International Journal of Innovative Computing, Information and Control. 2009;5:1643-1653.

[382] Samareh Moosavi SH, Bardsiri VK. Poor and rich optimization algorithm: A new human-based and multi populations algorithm. Engineering Applications of Artificial Intelligence. 2019;86:165-181.

[383] Zhang J, Xiao M, Gao L, Pan Q. Queuing search algorithm: A novel metaheuristic algorithm for solving engineering optimization problems. Applied Mathematical Modelling. 2018;63:464 - 490.

[384] Shabani A, Asgarian B, Gharebaghi SA, Salido MA, Giret A. A new optimization algorithm based on search and rescue operations. Mathematical Problems in Engineering. 2019;2019:1-24.

[385] Ray T, Liew KM. Society and Civilization: An Optimization Algorithm Based on the Simulation of Social Behavior. IEEE Transactions On Evolutionary Computation. 2003;7(4):386-396.

[386] Xie XF, Zhang WJ, Yang ZL. Social cognitive optimization for nonlinear programming problems. vol. 2; 2002. p. 779 $-783$. 
[387] Wei Z, Cui Z, Zeng J. Social Cognitive Optimization Algorithm with Reactive Power Optimization of Power System. In: 2010 International Conference on Computational Aspects of Social Networks; 2010. p. 11-14.

[388] Xu Y, Cui Z, Zeng J. Social Emotional Optimization Algorithm for Nonlinear Constrained Optimization Problems. In: Panigrahi BK, Das S, Suganthan PN, Dash SS, editors. Swarm, Evolutionary, and Memetic Computing; 2010. p. $583-590$.

[389] Weibo W, Quanyuan F, Yongkang Z. A novel particle swarm optimization algorithm with stochastic focusing search for real-parameter optimization. In: 2008 11th IEEE Singapore International Conference on Communication Systems; 2008. p. 583-587.

[390] Dwi Purnomo H. Soccer Game Optimization: Fundamental Concept. Jurnal Sistem Komputer. 2012;4(1):25-36.

[391] Das B, Mukherjee V, Das D. Student psychology based optimization algorithm: A new population based optimization algorithm for solving optimization problems. Advances in Engineering Software. 2020;146(102804):1-17.

[392] Rashid MFFA. Tiki-taka algorithm: a novel metaheuristic inspired by football playing style. Engineering Computations. 2020;38:313-343.

[393] Mahmoodabadi MJ, Rasekh M, Zohari T. TGA: Team game algorithm. Future Computing and Informatics Journal. 2018;3(2):191-199.

[394] Rao RV, Savsani VJ, Vakharia DP. Teaching-learning-based optimization: A novel method for constrained mechanical design optimization problems. Computer-Aided Design. 2011;43(3):303-315.

[395] Bagheri H, Ara AL, Hosseini R. Thieves and Police, a New Optimization Algorithm: Theory and Application in Probabilistic Power Flow. IETE Journal of Research. 2021;67(6):951-968.

[396] Kaveh A, Zolghadr A. A Novel Meta-Heuristic Algorithm: Tug Of War Optimization. International Journal Of Optimization In Civil Engineering. 2014;6(4):469-492.

[397] Ardjmand E, Amin-Naseri MR. Unconscious Search - A New Structured Search Algorithm for Solving Continuous Engineering Optimization Problems Based on the Theory of Psychoanalysis. In: Advances in Swarm Intelligence; 2012. p. 233-242.

[398] Moghdani R, Salimifard K. Volleyball Premier League Algorithm. Applied Soft Computing. 2018;64:161 - 185.

[399] Yampolskiy RV, EL-Barkouky A. Wisdom of artificial crowds algorithm for solving NP-hard problems. International Journal of Bio-Inspired Computation. 2011;3(6):358-369. 
[400] Ghaemi M, Feizi-Derakhshi MR. Forest Optimization Algorithm. Expert Systems with Applications. 2014;41(15):6676-6687.

[401] Cheng L, Wu Xh, Wang Y. Artificial Flora (AF) Optimization Algorithm. Applied Sciences. 2018 02;8:329.

[402] Zhao Z, Cui Z, Zeng J, Yue X. Artificial Plant Optimization Algorithm for Constrained Optimization Problems. In: 2011 Second International Conference on Innovations in Bio-inspired Computing and Applications; 2011. p. $120-123$.

[403] Ghaemidizaji M, Dadkhah C, Leung H. A new optimization algorithm based on the behavior of BrunsVigia flower. In: 2018 IEEE International Conference on Systems, Man, and Cybernetics (SMC). IEEE; 2018. p. 263-267.

[404] Yang XS. Flower Pollination Algorithm for Global Optimization. In: Unconventional Computation and Natural Computation, Proceeding; 2012. p. 240-249.

[405] Moez H, Kaveh A, Taghizadieh N. Natural Forest Regeneration Algorithm: A New Meta-Heuristic. Iranian Journal of Science and Technology, Transactions of Civil Engineering. 2016 Dec;40(4):311-326.

[406] Cai W, Yang W, Chen X. A Global Optimization Algorithm Based on Plant Growth Theory: Plant Growth Optimization. In: 2008 International Conference on Intelligent Computation Technology and Automation (ICICTA). vol. 1; 2008. p. 1194-1199.

[407] Sulaiman M, Salhi A, Selamoglu BI, Kirikchi OB. A Plant Propagation Algorithm for Constrained Engineering Optimisation Problems. Mathematical Problems in Engineering. 2014;2014:1-10.

[408] Premaratne U, Samarabandu J, Sidhu T. A new biologically inspired optimization algorithm. In: 2009 International Conference on Industrial and Information Systems (ICIIS); 2009. p. 279-284.

[409] Xiaoxian He, Shigeng Zhang, Jie Wang. A novel algorithm inspired by plant root growth with self-similarity propagation. In: 2015 1st International Conference on Industrial Networks and Intelligent Systems (INISCom); 2015. p. $157-162$.

[410] Labbi Y, ben attous D, A Gabbar H, Mahdad B, Zidan A. A new rooted tree optimization algorithm for economic dispatch with valve-point effect. International Journal of Electrical Power \& Energy Systems. 2016 07;79:298-311.

[411] Merrikh-Bayat F. The runner-root algorithm: A metaheuristic for solving unimodal and multimodal optimization problems inspired by runners and roots of plants in nature. Applied Soft Computing. 2015;33:292 - 303.

[412] Karci A. Theory of Saplings Growing Up Algorithm. In: Adaptive and Natural Computing Algorithms; 2007. p. $450-460$. 
[413] Caraveo C, Valdez F, Castillo O. A new optimization meta-heuristic algorithm based on self-defense mechanism of the plants with three reproduction operators. Soft Computing. 2018;22(15):4907-4920.

[414] Merrikh-Bayat F. A Numerical Optimization Algorithm Inspired by the Strawberry Plant; 2014.

[415] Cheraghalipour A, Hajiaghaei-Keshteli M, Paydar MM. Tree Growth Algorithm (TGA): A novel approach for solving optimization problems. Engineering Applications of Artificial Intelligence. 2018;72:393 - 414.

[416] Halim H, Ismail I. Tree Physiology Optimization in Constrained Optimization Problem. Telkomnika (Telecommunication Computing Electronics and Control). 2018 04;16:876-882.

[417] Kiran MS. TSA: Tree-seed algorithm for continuous optimization. Expert Systems with Applications. 2015;42(19):6686 - 6698.

[418] Punnathanam V, Kotecha P. Yin-Yang-pair Optimization: A novel lightweight optimization algorithm. Engineering Applications of Artificial Intelligence. 2016;54:62-79.

[419] Gao-Wei Y, Zhanju H. A Novel Atmosphere Clouds Model Optimization Algorithm. In: 2012 International Conference on Computing, Measurement, Control and Sensor Network; 2012. p. 217-220.

[420] Civicioglu P. Artificial cooperative search algorithm for numerical optimization problems. Information Sciences. 2012;229:58-76.

[421] Pijarski P, Kacejko P. A new metaheuristic optimization method: the algorithm of the innovative gunner (AIG). Engineering Optimization. 2019;51(12):1-21.

[422] Wu G. Across neighborhood search for numerical optimization. Information Sciences. 2016;329:597-618.

[423] Rahkar Farshi T. Battle royale optimization algorithm. Neural Computing and Applications. 2021;33(4):1139-1157.

[424] Del Acebo E, De La Rosa JL. Introducing bar systems: A class of swarm intelligence optimization algorithms. In: AISB 2008 Convention: Communication, Interaction and Social Intelligence - Proceedings of the AISB 2008 Symposium on Swarm Intelligence Algorithms and Applications; 2008. p. 18-23.

[425] Civicioglu P. Backtracking Search Optimization Algorithm for numerical optimization problems. Applied Mathematics and Computation. 2012;219(15):8121-8144.

[426] Zhu C, Ni J. Cloud Model-Based Differential Evolution Algorithm for Optimization Problems. In: 2012 Sixth International Conference on Internet Computing for Science and Engineering; 2012. p. 55-59. 
[427] Li B, Jiang W. Optimizing complex functions by chaos search. Cybernetics and Systems. 1998;29(4):409-419.

[428] Nunes de Castro L, Von Zuben FJ. The Clonal Selection Algorithm with Engineering Applications. In: Workshop Proceedings of GECCO. vol. 10; 2000. p. 36-37.

[429] Hosseini E, Ghafoor KZ, Sadiq AS, Guizani M, Emrouznejad A. COVID-19 Optimizer Algorithm, Modeling and Controlling of Coronavirus Distribution Process. IEEE Journal of Biomedical and Health Informatics. 2020;24(10):2765-2775.

[430] DEHGHANI M, MONTAZERI Z, MALIK OP. DGO: Dice game optimizer. Gazi University Journal of Science. 2019;32(3):871-882.

[431] Kadioglu S, Sellmann M. Dialectic Search. In: Gent IP, editor. Principles and Practice of Constraint Programming CP 2009. Berlin, Heidelberg: Springer Berlin Heidelberg; 2009. p. 486-500.

[432] Civicioglu P. transforming geocentric cartesian coordinates to geodetic coordinates by using differential search algorithm. Computers and Geosciences. 2012;46:229-247.

[433] Ghorbani N, Babaei E. Exchange market algorithm. Applied Soft Computing. 2014;19:177-187.

[434] Boettcher S, Percus AG. Extremal Optimization: Methods Derived from Co-evolution. In: Proceedings of the 1st Annual Conference on Genetic and Evolutionary Computation - Volume 1. GECCO’99; 1999. p. 825-832.

[435] Tan Y, Zhu Y. Fireworks Algorithm for Optimization. In: Tan Y, Shi Y, Tan KC, editors. Advances in Swarm Intelligence; 2010. p. 355-364.

[436] Shayanfar H, Gharehchopogh FS. Farmland fertility: A new metaheuristic algorithm for solving continuous optimization problems. Applied Soft Computing. 2018;71:728-746.

[437] Ahrari A, Atai AA. Grenade Explosion Method-A novel tool for optimization of multimodal functions. Applied Soft Computing. 2010;10:1132-1140.

[438] Tanyildizi E, Demir G. Golden sine algorithm: a novel math-inspired algorithm. Advances in Electrical and Computer Engineering. 2017;17(2):71-79.

[439] Hatamlou A. Heart: a novel optimization algorithm for cluster analysis. Progress in Artificial Intelligence. 2014;2(2):167-173. 
[440] Sellmann M, Tierney K. Hyper-parameterized Dialectic Search for Non-linear Box-Constrained Optimization with Heterogenous Variable Types. In: Kotsireas IS, Pardalos PM, editors. Learning and Intelligent Optimization. Cham: Springer International Publishing; 2020. p. 102-116.

[441] Hosseini SH, Ebrahimi A. Ideological Sublations: Resolution of Dialectic in Population-based Optimization; 2017.

[442] Gandomi AH. Interior search algorithm (ISA): A novel approach for global optimization. ISA Transactions. 2014;53(4):1168-1183.

[443] Hajiaghaei-Keshteli M, Aminnayeri M. Solving the integrated scheduling of production and rail transportation problem by Keshtel algorithm. Applied Soft Computing. 2014;25:184-203.

[444] Jaddi NS, Alvankarian J, Abdullah S. Kidney-inspired algorithm for optimization problems. Communications in Nonlinear Science and Numerical Simulation. 2017;42:358-369.

[445] De Melo VV. Kaizen Programming. In: Proceedings of the 2014 Annual Conference on Genetic and Evolutionary Computation. GECCO '14; 2014. p. 895-902.

[446] Nishida TY. In: Membrane Algorithms: Approximate Algorithms for NP-Complete Optimization Problems. Springer Berlin Heidelberg; 2006. p. 303-314.

[447] Sadollah A, Bahreininejad A, Eskandar H, Hamdi M. Mine blast algorithm: A new population based algorithm for solving constrained engineering optimization problems. Applied Soft Computing. 2013;13(5):2592-2612.

[448] Asil Gharebaghi S, Ardalan Asl M. New meta-heuristic optimization algorithm using neuronal communication. Iran University of Science \& Technology. 2017;7(3):413-431.

[449] Chan CY, Xue F, Ip W, Cheung C. A hyper-heuristic inspired by pearl hunting. In: International Conference on Learning and Intelligent Optimization. Springer; 2012. p. 349-353.

[450] Savsani P, Savsani V. Passing vehicle search (PVS): A novel metaheuristic algorithm. Applied Mathematical Modelling. 2016;40(5-6):3951-3978.

[451] Ansótegui C, Pon J, Sellmann M, Tierney K. Reactive Dialectic Search Portfolios for MaxSAT. In: Proceedings of the Thirty-First AAAI Conference on Artificial Intelligence. AAAI'17. AAAI Press; 2017. p. 765-772.

[452] Felipe D, Goldbarg EFG, Goldbarg MC. Scientific algorithms for the Car Renter Salesman Problem. In: 2014 IEEE Congress on Evolutionary Computation (CEC); 2014. p. 873-879. 
[453] Fathollahi-Fard AM, Hajiaghaei-Keshteli M. Social Engineering Optimization (SEO), A New Single-Solution Meta-heuristic Inspired by Social Engineering; 2017. .

[454] Salimi H. Stochastic Fractal Search: A powerful metaheuristic algorithm. Knowledge-Based Systems. 2015;75:1-18.

[455] Gonçalves MS, Lopez RH, Fadel Miguel LF. Search group algorithm: A new metaheuristic method for the optimization of truss structures. Computers and Structures. 2015;153:165-184.

[456] Hasançebi O, Azad SK. An efficient metaheuristic algorithm for engineering optimization: SOPT. International Journal of Optimization in Civil Engineering. 2012;2(4):479-487.

[457] Du H, Wu X, Zhuang J. Small-World Optimization Algorithm for Function Optimization. In: Advances in Natural Computation; 2006. p. 264-273.

[458] Dueck G. New optimization heuristics; The great deluge algorithm and the record-to-record travel. Journal of Computational Physics. 1993;104(1):86-92.

[459] Bayraktar Z, Komurcu M, Werner DH. Wind Driven Optimization (WDO): A novel nature-inspired optimization algorithm and its application to electromagnetics. In: 2010 IEEE Antennas and Propagation Society International Symposium; 2010. p. 1-4.

[460] Sörensen K. Metaheuristics - the metaphor exposed. International Transactions in Operational Research. 2015;22:3-18.

[461] García-Martínez C, Gutiérrez PD, Molina D, Lozano M, Herrera F. Since CEC 2005 competition on real-parameter optimisation: a decade of research, progress and comparative analysis's weakness. Soft Computing. 2017;21(19):5573-5583.

[462] Liao T, Molina D, Stützle T. Performance evaluation of automatically tuned continuous optimizers on different benchmark sets. Applied Soft Computing. 2015;27:490-503.

[463] Bosman PAN, Gallagher M. The importance of implementation details and parameter settings in black-box optimization: a case study on Gaussian estimation-of-distribution algorithms and circles-in-a-square packing problems. Soft Computing. 2018 Feb;22(4):1209-1223.

[464] Biedrzycki R. On Equivalence of Algorithm's Implementations: The CMA-ES Algorithm and Its Five Implementations. In: Proceedings of the Genetic and Evolutionary Computation Conference Companion. GECCO '19. New York, NY, USA: Association for Computing Machinery; 2019. p. 247-248. 
[465] Liefooghe A, Jourdan L, Talbi EG. A software framework based on a conceptual unified model for evolutionary multiobjective optimization: ParadisEO-MOEO. European Journal of Operational Research. 2011;209(2):104-112.

[466] Durillo JJ, Nebro AJ. jMetal: A Java framework for multi-objective optimization. Advances in Engineering Software. 2011;42(10):760 - 771.

[467] Vrbančič G, Brezočnik L, Mlakar U, Fister D, Fister Jr I. NiaPy: Python microframework for building nature-inspired algorithms. Journal of Open Source Software. 2018;3.

[468] Benítez-Hidalgo A, Nebro AJ, García-Nieto J, Oregi I, Ser JD. jMetalPy: A Python framework for multi-objective optimization with metaheuristics. Swarm and Evolutionary Computation. 2019;51:100598.

[469] Tian Y, Cheng R, Zhang X, Jin Y. PlatEMO: A MATLAB platform for evolutionary multi-objective optimization. IEEE Computational Intelligence Magazine. 2017;12(4):73-87.

[470] Gupta A, Ong Y. Memetic Computation: The Mainspring of Knowledge Transfer in a Data-Driven Optimization Era. Adaptation, Learning, and Optimization Series, Springer; 2018.

[471] Mafarja M, Qasem A, Heidari AA, Aljarah I, Faris H, Mirjalili S. Efficient Hybrid Nature-Inspired Binary Optimizers for Feature Selection. Cognitive Computation. 2019 Jul;p. 1-26. 